\title{
Partizipation in \\ Umweltorganisationen
}

Eine vergleichende qualitative Untersuchung anhand zweier NGOs in Göttingen

von Moritz Engbers

aus Oldenburg

Georg-August-Universität Göttingen

Institut für Geographie

Abteilung Humangeographie

Matrikelnummer: 20415335

Erstgutachter: Prof. Dr. Heiko Faust

Zweitgutachter: Prof. Dr. Werner Kreisel

Göttingen, September 2010 


\section{Danksagung}

Hiermit möchte ich mich ganz herzlich bei allen bedanken, die mich bei der Themenwahl, während des Schreibens und bei den Abschlusskorrekturen unterstützt haben. Ohne die vielen Diskussionen, Aufmunterungen und Anmerkungen wäre diese Arbeit sicher nicht zu Stande gekommen. Ein besonderer Dank geht auch an meine Eltern und meinen Bruder, die während meines Studiums stets auf unterschiedlichste Art und Weise für mich da waren. Schließlich möchte ich mich noch herzlich bei meinen Interviewpartnerinnen und Interviewpartnern bedanken, die bereit waren, ihre Erfahrungen mit mir zu Teilen. 


\section{Inhaltsverzeichnis}

$\begin{array}{lll}1 & \text { Einleitung } & 1\end{array}$

1.1 Ziel der Arbeit . . . . . . . . . . . . . . . . . . . . . . . . . . . . . 1

1.2 Forschungsstand . . . . . . . . . . . . . . . . . . . . 2

1.3 Forschungsfrage . . . . . . . . . . . . . . . . . . . . . 3

1.4 Aufbau der Arbeit . . . . . . . . . . . . . . . . . . . . . . . 4

2 Umweltbewegungen und -organisationen in Deutschland 5

$2.1 \quad$ Nichtregierungsorganisationen . . . . . . . . . . . . . . 5

2.2 Soziale Bewegungen . . . . . . . . . . . . . . . . . . . . . 7

$2.3 \quad$ Konzepte von Natur und Umwelt . . . . . . . . . . . . . . . . 8

2.4 Umwelteinstellung und -verhalten . . . . . . . . . . . . . . 8

2.5 Die Umwelt- und Naturschutzbewegungen in Deutschland. . . . . . . 9

$2.5 .1 \quad$ Vom 19. Jahrhundert bis nach dem Zweiten Weltkrieg. . . . . 9

$2.5 .2 \quad 1960 \mathrm{er}$ bis Anfang der 1980er Jahre . . . . . . . . . . . . . . . 11

2.5 .3 1980er und 1990er Jahre . . . . . . . . . . . . . 12

$2.5 .4 \quad$ Jüngste Entwicklungen . . . . . . . . . . . . . . . . . . . . . . 13

2.5 .5 Strukturen, Strategien und Perspektiven . . . . . . . . . . . . 14

2.5 .6 Ausblick . . . . . . . . . . . . . . . . . . . . . . 16

2.6 Zusammenfassung . . . . . . . . . . . . . . . . . . . . . . . . 17

3 Partizipation 18

3.1 Begriffsbestimmung . . . . . . . . . . . . . . . . . . . . . . . 18

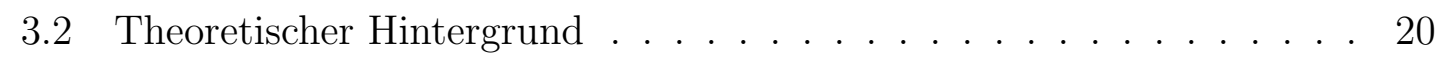

$3.2 .1 \quad$ Zivilgesellschaft . . . . . . . . . . . . . . . . . 20

3.2 .2 Partizipationstheorien . . . . . . . . . . . . . . . . . . 25

3.3 Kritische Überlegungen zum Partizipationsbegriff . . . . . . . . . . . . 27

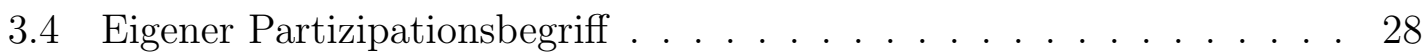

3.5 Zusammenfassung . . . . . . . . . . . . . . . . . . . . . . . 30

4 Forschungsdesign und Methodik 31

4.1 Theoretische Perspektive und Prämissen . . . . . . . . . . . . . . . . 31

$4.1 .1 \quad$ Vorteile qualitativer Forschung. . . . . . . . . . . . . . . . 31 
4.1 .2 Aufstellen von Hypothesen . . . . . . . . . . . . . . . . . . . . 32

4.1 .3 Gütekriterien . . . . . . . . . . . . . . . . . . . . . . 33

4.2 Forschungsdesign $\ldots \ldots \ldots \ldots$. . . . . . . . . . . . . . . . . . 34

4.2 .1 Ziele der Untersuchung . . . . . . . . . . . . . . . . . . 34

$4.2 .2 \quad$ Fragestellung und Hypothesen . . . . . . . . . . . . . . . . 34

4.2 .3 Fallauswahl . . . . . . . . . . . . . . . . . . . 36

4.2 .4 Erhebungsmethode . . . . . . . . . . . . . . . . . . . . . . . . . . . . . . . . . . . . . .

4.2 .5 Aufbereitungsverfahren . . . . . . . . . . . . . . . . . . . . 38

4.2 .6 Auswertungsverfahren $\ldots \ldots \ldots \ldots$

4.3 Zusammenfassung . . . . . . . . . . . . . . . . . . . . . . . . 43

$\begin{array}{lll}5 & \text { Empirie } & 44\end{array}$

$5.1 \quad$ Beschreibung der untersuchten Organisationen . . . . . . . . . . . . . 44

5.1 .1 Greenpeace . . . . . . . . . . . . . . . . . 44

5.1 .2 Deutscher Jungendbund für Naturbeobachtung . . . . . . . . 47

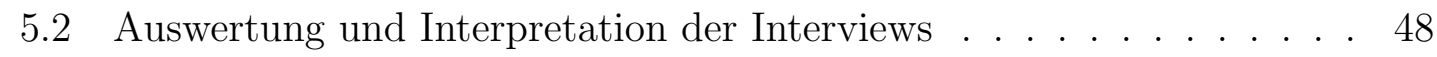

$5.2 .1 \quad$ Die Organisationen . . . . . . . . . . . . . . . . . . . . . . . 49

5.2 .2 Motivation . . . . . . . . . . . . . . 53

5.2 .3 Partizipation $\ldots \ldots \ldots \ldots \ldots$

5.2 .4 Auswirkungen des Engagements . . . . . . . . . . . . . . 64

5.2 .5 Zusammenarbeit ................... . . 66

5.2 .6 Altersstruktur . . . . . . . . . . . . . . . . . 71

5.2 .7 Bedeutung des Internets . . . . . . . . . . . . . . 73

5.2 .8 Austausch zwischen Gruppen . . . . . . . . . . . . . . . . . . 75

5.3 Zusammenfassung der Ergebnisse . . . . . . . . . . . . . . 76

\begin{tabular}{lll}
\hline 6 & Diskussion & 79
\end{tabular}

$6.1 \quad$ Einordnung in Umweltbewegungen . . . . . . . . . . . . . . . . . . . 79

6.2 Einordnung in Zivilgesellschafts- und Partizipationstheorie . . . . . . 81

$6.3 \quad$ Forschungsfrage und Hypothesen . . . . . . . . . . . . . . . . . 82

7 Reflexion und Fazit $\quad 86$

7.1 Inhaltliche und theoretische Reflexion . . . . . . . . . . . . . . . . . 86

7.2 Methodische Reflexion . . . . . . . . . . . . . . . . . . . . . . 87

7.3 Fazit und Ausblick . . . . . . . . . . . . . . . . . . . . . . . . . . . 87

\begin{tabular}{llr}
\hline 8 & Literatur & 89
\end{tabular}

A Anhang

A.1 Interviewleitfaden „Partizipation in Umweltorganisationen ${ }^{\prime}$. . . . . . I I

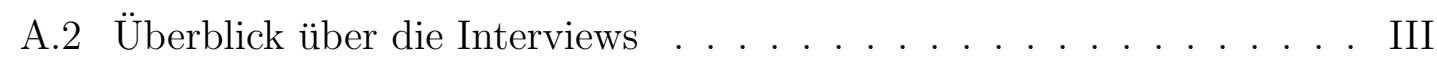


A.3 Inhaltsverzeichnis $\operatorname{der} \mathrm{CD} \ldots \ldots \ldots \ldots$. . . . . . . . . . . 


\section{Abkürzungsverzeichnis}

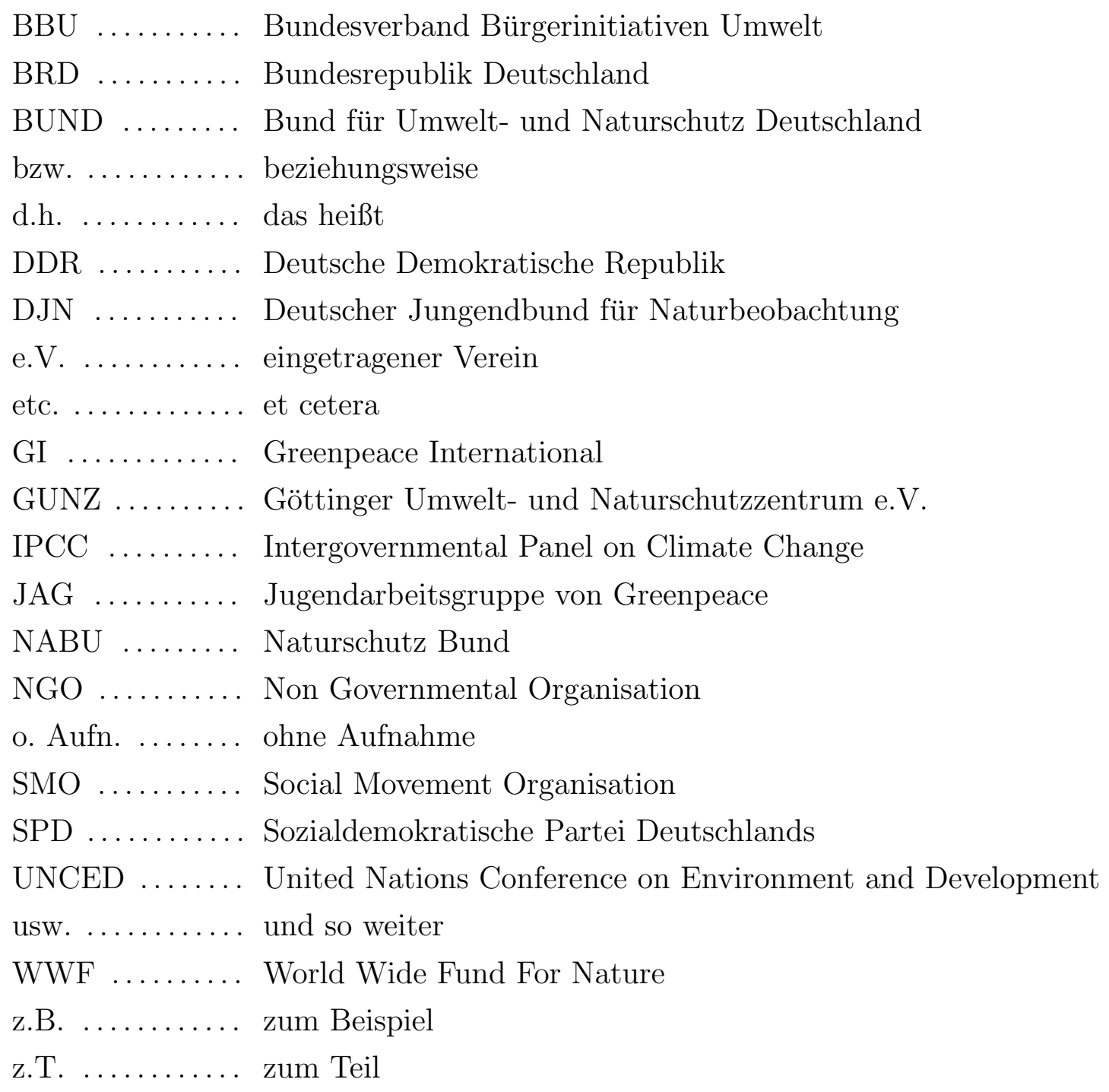




\section{Einleitung}

Die deutsche Umweltbewegung ist vielen Veränderungen ausgesetzt: Seit den 1990er Jahren sind die Themen Nachhaltigkeit und Klimawandel immer stärker in die öffentliche Aufmerksamkeit getreten. Zugleich ändert sich die Komplexität und die Zahl der Themen. Viele Strategien des Umwelt- und Naturschutzes haben sich vom Beginn des 20. Jahrhunderts, über die 1960er und 1970er Jahren bis heute gewandelt. Zugleich verändert sich die Rolle von Umwelt- und Naturschutzorganisationen und ihr Verhältnis zu Staat, Wirtschaft und ihren Mitgliedern. Umweltorganisationen bewegen sich in einem Feld aus zum einen umweltpolitischen und naturschutzorientierten Themen und zum anderen aus zivilgesellschaftlichen Trends.

Dies schließt auch die Partizipation in Umweltorganisationen, also die freiwillige Beteiligung und Zusammenarbeit, ein. Diesem Phänomen widmet sich diese Arbeit: Anhand von zwei Umweltorganisationen sollen die Voraussetzungen für und die Umsetzung von Partizipation untersucht werden. Die Betrachtung von Motiven, Motivationen, Formen der Zusammenarbeit und Strategien kann helfen, die aktuellen Entwicklungen im Bereich der Umweltorganisationen mit ihren Auswirkungen in umweltpolitischen Themenfeldern, in ihrer kommunalen Bedeutung und dem gesellschaftlichen Verhältnis zu Umwelt- und Naturschutz besser zu verstehen. Außerdem ergibt sich daraus die Möglichkeit, Gruppenprozesse in Bezug auf Mitwirkung und Entscheidungsfindung zu untersuchen. Im Gegensatz zu vielen quantitativen Studien in diesem Bereich geht die Arbeit einen qualitativen Weg: Durch Interviews mit Partizipierenden wurden konkrete Erfahrungen und Deutungsmuster untersucht, die zu einem Mitwirken in Umweltorganisationen geführt haben. Der Ansatz ist dabei ein explorativer, d.h. es werden (im Abgleich mit theoretischen Konzepten) neue Zusammenhänge erschlossen, um sich dem „Verstehen“ durch konkrete Einzelfälle zu nähern.

\subsection{Ziel der Arbeit}

Ziel dieser Arbeit ist es, Partizipation in Umweltorganisationen zu untersuchen. Dies beinhaltet das Verständnis von Partizipation, sowie die Motivationen und Ziele für die Beteiligung. Dadurch lassen sich Aussagen für verschiedene Bereiche treffen: Zum einen ermöglicht sie ein besseres Verständnis der zivilgesellschaftlichen Prozesse, die 
$\mathrm{zu}$ einer aktiven Partizipation in Umweltorganisationen führen und zum anderen wird es möglich, Aussagen darüber zu treffen, wie Umwelt und Umweltkonflikte in den Organisationen diskutiert werden und welche Ziele sich daraus ableiten. Des Weiteren stellt Partizipation in vielen Bereichen eine wichtige Grundlage für Entscheidungsfindungen und Handlungen dar. Die Erkenntnisse lassen sich z.B. auf Felder, wie Partizipation in der Stadtplanung oder partizipatives Ressourcenmanagement, übertragen. Diese Arbeit hat einen explorativen Charakter, das heißt aufgrund der theoretischen Vorüberlegungen und der angewandten qualitativen Methodik wird, neben der Überprüfung von Hypothesen, auch Wert auf die Entwicklung neuer Hypothesen gelegt und kann als Vorstudie für weitere Forschungstätigkeit in diesem Bereich dienen. Diese Arbeit hat einen explizit methodischen Fokus: Aus diesem Grund nimmt die Darstellung des Forschungsdesigns und der Methodik einen breiteren Raum ein. Auf theoretischer Ebene dient diese Arbeit dazu, bestehende Konzepte zu vertiefen. Außerdem ist sie auch für PraktikerInnen interessant, die sich mit der Partizipation bzw. der Erhöhung der Partizipation in (Umwelt)Organisationen befassen. Die Arbeit befindet sich somit an der Schnittstelle zwischen Sozialgeographie und Umweltsoziologie. Eine detailliertere Darstellung der Ziele der Untersuchung finden sich im Kapitel Forschungsdesign und Methodik (4).

\subsection{Forschungsstand}

Allgemein dominieren quantitative Untersuchungen das Thema zivilgesellschaftliches Engagement und Partizipation. Diese reichen von Telefoninterviews und Erhebungen mit Fragebögen über Auswertungen innerhalb von großen Panelstudien bis hin zu internationalen Großprojekten, die an den World Values Survey angelehnt sind (z.B. Bekkers 2005, Cohn, Barkan \& Halteman 2003, EnquetteKommission 2002, Freeman 1997, Martinez \& McMullin 2004, Torgler \& Garcías-ValinÑas 2006). Bekkers (2005) weist darauf hin, dass es zwar viele Studien in Fächern wie Soziologie, Politikwissenschaft, Psychologie oder Wirtschaftswissenschaften gibt, interdisziplinäre Perspektiven allerdings bisher größtenteils ausblieben. Dies betont auch Kersting (2008: 11), der Partizipation als „,interdisziplinäres Querschnittsthema“ bezeichnet. Zum Thema Umweltverhalten gibt es eine Vielzahl von Forschungsarbeiten, jedoch gebe es kaum Studien darüber, welche Faktoren Partizipation im Umweltbereich beeinflussen bzw. wie Partizipation praktisch „,funktioniert“ (TORGLER \& GARCIA-VAliñAS 2006: 3-4). Cohn, Barkan \& Halteman (2003) weisen darauf hin, dass viel zur Anwerbung von neuen Mitgliedern geforscht wurde, die Thematik des Haltens von Mitgliedern in der Literatur bisher kaum behandelt werde. Ebenso merken sie an, dass die Bedeutung von Biographien bisher eine black box in der Literatur über 
Organisationen darstellt. Stange (2008) führt an, dass in vielen Untersuchungen zudem häufig unklare oder sehr heterogene Auffassungen von Partizipation zu Grunde gelegt werden. Generell gibt es wenige qualitative Studien zur Partizipation: DAHM \& SCHERHORN (2008) legen eine quantitative und qualitative Untersuchung zu urbaner Subsistenz und bürgerschaftlichem Engagement vor. Die Studie von Mitlacher \& Schulte (2005) stellt eine Kombination aus qualitativen und quantitativen Methoden zur Steigerung des ehrenamtlichen Engagements in Naturschutzverbänden dar. Weitere Arbeiten beziehen sich hauptsächlich auf die Partizipation von Kindern und Jugendlichen. Beispiele sind hier Moser (2010) zur gesellschaftlichen Partizipation aus der Sicht von Jugendlichen, sowie Arbeiten der „grauen Literatur“, wie z.B. ZeILER \& Schell (2003), die zur Partizipation von Mädchen in Umweltorganisationen geforscht haben. Hier knüpft auch die vorliegende Arbeit an, die das Forschungsfeld der qualitativen Studien im Bereich der Partizipation erweitert. Konkrete Studien zum Deutschen Jungendbund für Naturbeobachtung (DJN) sind nicht bekannt. Zu Greenpeace ist grade eine quantitative Studie zum Management von Ehrenamtlichen erschienen (WALLRAFF 2010). Die aktuelleste Studie, eine quantitativ-ökonomische, die sich konkret mit der Partizipation in Umweltorganisationen beschäftigt, ist grade erschienen (TORGLER, Garcías-VALiñas \& Macintyre 2010).

\subsection{Forschungsfrage}

Aus dem Stand der Forschung ergibt sich die Forschungsfrage. Da es bisher wenige vergleichende und fast nur quantitative Studien gibt, wurde an diesem Punkt angesetzt und eine Forschungsfrage formuliert, die eine qualitative und vergleichede Ausrichtung hat. Die Forschungsfrage lautet:

Wie gestaltet sich Partizipation in den beiden Umweltorganisationen, DJN und Greenpeace in Göttingen, und gibt es Gemeinsamkeiten und Unterschiede zwischen ihnen?

Im Abschnitt Fragestellung und Hypothesen 4.2.2 werden die Forschungsfrage und sich daran anschließende Leitfragen detailierter diskutiert, um als Ausgangspunkt für die empirische Untersuchung zu dienen. Der Partizipationsbegriff der Fragestellung wird im Laufe der Arbeit mit gesellschaftstheoretischen Grundlagen unterfüttert und in einen größeren Zusammenhang mit der Entwicklung der Umweltbewegungen in Deutschland gebracht. 


\subsection{Aufbau der Arbeit}

Zunächst werden im Kapitel Umweltbewegungen und -organisationen in Deutschland (2) die Begriffe Nichtregierungsorganisation und soziale Bewegung diskutiert, darauf folgt ein kurzer Abriss der Geschichte der Umweltbewegung in der BRD (und in Teilen auch der DDR), um schließlich auf Perspektiven und aktuelle Entwicklungen einzugehen. Im Kapitel Partizipation (3), wird sich dem Konzept der Partizipation, zunächst über die Einordnung in das Feld der Zivilgesellschaft, theoretisch angenähert. Aus diesen Vorüberlegungen folgt dann der Einstieg in die Empirie. Nach einer Darstellung des Forschungsdesigns und der Auswahl der Methodik im Kapitel Forschungsdesign und Methodik (4), wird im empirischen Hauptteil, dem Kapitel Empirie (5), die Auswertung der Interviews aus den untersuchten Umweltorganisationen dargelegt. Im vorletzten Kapitel Diskussion (6) erfolgen dann eine Zusammenführung aus Theorie und Empirie, die Beantwortung der Forschungsfrage und die Diskussion der Hypothesen. Die Arbeit endet mit dem Kapitel Reflexion und Fazit (7), in dem eine inhaltliche, methodische und theoretische Reflexion des Forschungsprozesses stattfindet und das mit einem Fazit abschließt. 


\section{Umweltbewegungen und -organisationen in Deutschland}

In diesem Kapitel werden die Begriffe der „Organisation“ bzw. der „sozialen Bewegung“" näher betrachtet, da sie den theoretischen Hintergrund für die Entstehung und Entwicklung von Umweltorganisationen darstellen. Danach wird die Geschichte der Umweltorganisationen in Deutschland skizziert, um nachvollziehen zu können, welche Bedingungen zur Entstehung geführt haben, welche gesellschaftlichen Verhältnisse relevant und welche Vorstellung von Natur und Umwelt ausschlaggebend dafür waren. Das Kapitel schließt mit einem Ausblick auf mögliche zukünftige Entwicklungen der Umweltorganisationen. Diese Ausführungen sollen helfen, die untersuchten Umweltorganisationen in einen größeren Umweltdiskurs einzuordnen und ihre Ziele und Strategien in einen gesellschaftlichen Zusammenhang zu stellen. Wenn in dieser Arbeit von Umweltorganisationen gesprochen wird, dann sind damit Nichtregierungsorganisation gemeint, die in naturkundlichen oder umweltpolitischen Bereichen tätig sind.

\subsection{Nichtregierungsorganisationen}

Es gibt verschiedene Begriffe, die für zivilgesellschaftliche Organisationen verwendet werden. In der Literatur tauchen Begriffe wie Nichtregierungsorganisation, NonProfit-Organisation, Verein oder voluntary association auf. MARTEns (2002) fasst diese begriffliche Diskussion zusammen und stellt fest, dass es keine einheitlichen Definitionen für die einzelnen Begriffe gibt und sie größtenteils deckungsgleich verwendet werden. In der deutschen Fachliteratur ist es üblich, die englische Abkürzung NGO für non governmental organisation (Nichtregierungsorganisation) zu verwenden und der Ansatz wird auch in der dieser Arbeit verfolgt.

Nach Martens (2002) gibt es einen juristischen und einen soziologischen Ansatz, um sich dem Begriff der NGO zu nähern. Juristisch gesehen werden NGOs in Deutschland als Vereine behandelt. Dabei ist es freigestellt, ob sich ein Verein als „eingetragener Verein“ registrieren lassen möchte, damit er als juristische Person agieren kann ${ }^{1}$. In der Soziologie werden, laut MARTEns (2002), NGOs bezüglich

\footnotetext{
${ }^{1}$ Dies ist zum Beispiel in Großbritannien verpflichtend.
} 
der Wortschöpfung „Nichtregierungsorganisation“ (z.B. die negierende Abgrenzung zu Regierungsorganisationen), des Verhältnisses zu Staat und Wirtschaft oder der Form und Struktur der Organisationen, untersucht. HiRsch (2001: 15-16) definiert NGOs als auf nationaler und internationaler Ebene agierende, formell private Einrichtungen mit den Eigenschaften Gemeinnützigkeit, stellvertretende Interessenwahrnehmung, formelle organisatorische und finanzielle Unabhängigkeit von Staat und Unternehmen und eine gewisse Professionalisierung und organisatorische Dauerhaftigkeit (HIRSch 2001). DELBRÜCK (2003) ergänzt, dass NGOs ideelle Ziele verfolgen sollten, aber trotzdem über Einnahmen (z.B. durch Spenden) verfügen dürften.

Durch die Abgrenzung von Regierungsorganisationen werden dem Begriff der NGO keine Eigenschaften zugewiesen, sondern es wird lediglich definiert, welche Eigenschaften das Konzept nicht hat. Das ist zum einen unpräzise, zum anderen wird dadurch möglicherweise ein Normalzustand impliziert, ähnlich dem Begriff der non profit organisations, in dem Organisationen normalerweise der Regierung nahestehen bzw. gewinnmaximierend arbeiten. Des Weiteren werden „Gemeinnützigkeit“, „stellvertretende Interessenwahrnehmung“ und „ideelle Ziele“ angesprochen. Diese sind allerdings davon anhängig, wie NGOs bzw. deren Mitglieder und Führung diese interpretieren. Auch ist fraglich, wessen Interessen wahrgenommen werden sollen, es kann also nicht von einer reinen „Gemeinnützigkeit“ ausgegangen werden, da immer Interessen bestimmter Gruppen und auch der Organisation bzw. deren Führung vertreten werden.

Für die Analyse von Organisationen stellt Markham (2002) zwei grundlegende Perspektiven in den Sozialwissenschaften fest: zum einen die Betrachtung als Interessengruppe, also den Zusammenschluss von Menschen mit ähnlichen Interessen, um diese politisch durchzusetzen (darunter fallen z.B. auch Gewerkschaften oder Industrieverbände), und zum anderen den Ansatz von sozialen Bewegungsorganisationen, die sich aus sozialen Bewegungen (z.B. den Umweltbewegungen) gebildet haben. In einer neueren Monographie ergänzt MARKham (2008) die beiden Ansätze noch durch organisationstheoretische Überlegungen. In diesem Kapitel wird sich eher auf die Perspektive der sozialen Bewegungen beschränkt, da sich diese gut für eine geschichtliche Überblicksdarstellung eignen, ohne andere Ansätze völlig außer Acht zu lassen.

Organisationen agieren interessengeleitet. Die Frage ist, ob es sich dabei um eine Form von „öffentlichem Interesse“, im Sinne von größeren Teilen der Bevölkerung, handelt oder um die Interessen einer kleinen Gruppe von Menschen. Wie POLLAK (2004) generell für zivilgesellschaftliche Organisationen hervorhebt, gibt es aber auch Machtgefälle und hegemoniale Aushandlungsprozesse innerhalb von Organisationen. 


\subsection{Soziale Bewegungen}

Neben der Betrachtung der Organisation lassen sich Umweltorganisationen in einen Zusammenhang mit sozialen Bewegungen bringen. Diese lassen sich ganz allgemein als „collective attempts to promote or resist change in a society or group" definieren (Benford 1992: 1880). Della Porta \& Diani (1999: 140-142) geben die folgenden Kriterien für soziale Bewegungen an:

- eine dezentralisierte Struktur

- eine Betonung von Partizipation

- eine starke interne Solidarität

- eine charakteristische Form von Führung.

Diese Kriterien sind sehr allgemein gehalten, insbesondere die dezentralisierte Struktur und die Betonung von Partizipation ${ }^{2}$, Die innere Solidarität und Form der Führungen sind sehr pauschale Aussagen, die so wahrscheinlich nicht auf alle sozialen Bewegungen zutreffen und nicht in einheitlicher Form auftreten.

Da eine soziale Bewegung aus heterogenen und fragmentierten Teilen besteht, wird als etwas konkretere Abgrenzung der Begriff SMO, der social movement organization, verwendet. Eine SMO kann als komplexe Organisation verstanden werden, deren Ziele mit den Präferenzen einer sozialen Bewegung übereinstimmen (McCarthy \& Zald 1977). Der Begriff der SMO ermöglicht es also, Teile einer sozialen Bewegung konkreter zuzuordnen. Dies können zum Beispiel Umweltorganisationen, entwicklungspolitische Organisationen oder Frauenrechtsorganisationen sein. Allerdings merkt YEARLEY (2005: 17) kritisch an: „There is [...] no single criterion allowing us to explain which pressure groups can be associated with a large-scale social movement and which cannot." In diesem Sinne sind die Übergänge zwischen NGOs, SMOs und sozialen Bewegungen fließend. Es handelt sich eher um verschiedene Blickwinkel auf soziale Phänomene. Soziale Bewegungen sind also gesellschaftliche Kräfte, aus denen sich Organisationen entwicklen können. Dieses Hintergrundwissen ist wichtig, um die Entstehung von Umweltorganisationen verstehen zu können.

\footnotetext{
${ }^{2}$ Diese wird hier als Teilnahme an Aktivitäten, die mit einer sozialen Bewegung verbunden sind, verstanden.
} 


\subsection{Konzepte von Natur und Umwelt}

„Natur'3 und „Umwelt" sind vielfach verwendete Begriffe. Dennoch wird selten gefragt, was genau mit diesen Begriffen gemeint ist, wenn sie in verschiedenen Zusammenhängen verwendet werden. Eine naturalistische Betrachtung ist, wenn es um die Einbeziehung von gesellschaftlichen Verhältnissen geht, nicht mehr haltbar. „Natur", „Umwelt" und „Ökologie" sind in der Gesellschaft kommunizierte und konstruierte Begriffe (Görg 1999) und insofern bleibt zu fragen, nach welchen Regeln diese Begriffe konstruiert werden und zu welchem Zweck. Im Sinne von Görg (1999: 9) werden "Natur" und Gesellschaft in dieser Arbeit als „konstitutiv aufeinander verweisende Begriffe“ verstanden. Es geht also darum, die gesellschaftlichen Bedingungen für bestimmte Naturbegriffe in den Vordergrund zu rücken. Das bedeute nicht, dass keine materiell-stoffliche Grundlage von Gesellschaft besteht, so Görg (1999), sondern, dass es um die Analyse der Begrifflichkeit von "Natur" und „Umwelt“ im Verhältnis zur Gesellschaft gehe. Laut BLÜHdORN (2002) führt eine Pluralisierung von Naturbegriffen auch zu stärker fragmentierten und differenzierten Umweltbewegungen.

\subsection{Umwelteinstellung und -verhalten}

Wenn von Umweltorganisationen und -bewegungen die Rede ist, tauchen oft die Begriffe Umweltverhalten, Umwelteinstellung, Umweltbewusstsein, sowie die englischen Varianten environmental concern, environmental attitude oder environmental conciousness 4 auf. Auf diese Begriffsverwirungen weisen Diekmann \& PreisendörFER (2001) und BAukloh \& Roose (2002) ausdrücklich hin. Bei der Verwendung der Begriffe in verschiedenen Publikationen fällt weiter auf, dass selten expliziert wird, was dabei konkret unter „Umwelt" verstanden wird. In dieser Arbeit soll auf eine Übersicht (Abb. 2.1) von BAUKLOH \& Roose (2002) verwiesen werden. In der Übersicht wird zum einen zwischen dem Umweltverhalten (environmental behaviour), also dem konkreten umweltfreundlichen ${ }^{5}$ Handeln in einer bestimmten Situation, und der Umwelteinstellung (general attitude), welche eher die generelle Einstellung bezeichnet, zwischen verschiedenen Handlungsalternativ-

\footnotetext{
${ }^{3}$ Die Begriffe „Natur“ und „Umwelt" werden in den meisten Fällen in dieser Arbeit in Anführungszeichen gesetzt, um ihre Begrifflichkeit hervorzuheben und sich von naturalistischen Vorstellungen abzugrenzen. Eine Begründung dafür erfolgt in diesem Kapitel. Das gleiche gilt für verwandte Begriffe, wie Naturschutz oder Umweltorganisation, aber aus Gründen der Lesbarkeit wird in den meisten Fällen auf Anführungszeichen verzichtet.

${ }^{4}$ Diese Begriffe lassen sich nicht immer direkt ins Deutsche übersetzen.

${ }^{5}$ Umweltfreundlich soll sich in diesem Zusammenhang auf Maßnahmen wie Recycling, Verzicht auf Autofahren oder gezieltere Wahl von Produkten, etc. beziehen. Da „Umwelt" keine eigene Entität ist, sondern eher eine Wahrnehmung darstellt, ist schwer zu differenzieren, ob etwas „freundlich“ ist, oder nicht.
} 


\begin{tabular}{|c|c|c|}
\hline & Einstellung & Verhalten \\
\hline \multirow[t]{2}{*}{ Alltagshandlungen } & pro-environmental attitude & pro-environmental behaviour \\
\hline & $\begin{array}{l}\text { - Unterstützung von Umweltschutz } \\
\text { als allgemeines Anliegen }\end{array}$ & $\begin{array}{l}\text { - Umweltfreundliches Verhalten und } \\
\text { Konsumption }\end{array}$ \\
\hline \multirow[t]{2}{*}{ politische Teilhabe } & political support of the environment & $\begin{array}{l}\text { political participation on behalf of the } \\
\text { environment }\end{array}$ \\
\hline & $\begin{array}{l}\text { - Unterschtützung von } \\
\text { Umweltschutz als politisches Ziel }\end{array}$ & $\begin{array}{l}\text { - wählen von umweltorientierten } \\
\text { Parteien } \\
\text { - Protest (im weiteren Sinne) } \\
\text { - Partizipation in Umweltorganisationen }\end{array}$ \\
\hline
\end{tabular}

Abbildung 2.1: Umwelteinstellung und -verhalten (nach BAUKLOH UND RoOsE 2002: 83, verändert)

en eine auszuwählen 6 , unterschieden. Ferner wird zwischen alltäglichen Handlungen (everyday activities) und politischer Aktivität (political participation), im Sinne einer gezielteren Auseinandersetzung mit den Themen, unterschieden. Diese Übersicht kann eher als allgemeiner Hintergrund gesehen werden an dem sich orientiert wird. Als analytische Kategorien sind sie aber unzureichend.

\subsection{Die Umwelt- und Naturschutzbewegungen in Deutschland}

In diesem Kapitel wird auf die Geschichte der Umweltbewegungen in Deutschland eingegangen, damit die untersuchten Umweltorganisationen in einen größeren Zusammenhang eingeordnet werden können. Dabei sollen nur einige markante Eckpunkte der deutschen Umweltpolitik und in diesem Zusammenhang auch der sozialen Bewegungen diskutiert werden, um einen Überblick zu gewinnen, aber den Rahmen der Arbeit nicht zu sprengen. Ein Schwerpunkt liegt dabei auf dem jeweiligen Verständnis von "Umwelt" und "Natur“, dass in den entsprechenden Zeiten dominant war und das heutige Denken im Umwelt- und Naturschutz nach wie vor beeinflusst. Ferner wird sich größtenteils auf eine Darstellung der westdeutschen Entwicklung beschränkt.

\subsubsection{Vom 19. Jahrhundert bis nach dem Zweiten Weltkrieg}

Seit Mitte des 19. Jahrhunderts begann sich in Deutschland ein Denkmal- und Naturschutz, der an die Gedanken der Romantik angelehnt war, zu etablieren. Die zunehmende Industrialisierung führte insbesondere bei GroßstädterInnen zu

\footnotetext{
${ }^{6}$ Es wird davon ausgegangen, dass Menschen zwar häufig behaupten sich umweltfreundlich zu verhalten, dies aber praktisch nicht tun.
} 
einer gefühlten Entfremdung von der "Natur“, so dass sich der Fokus der frühen Naturschutzbestrebungen auf ein romantisiertes und emotionalisiertes Bild von "Natur" bezog. Dieser Trend verstärkte sich noch, als in den 1880er Jahren die Folgen der Industrialisierung für die "Umwelt" zunehmend sichtbarer wurden (BRAND 2008). Daraus entwickelte sich eine Vielzahl von Bewegungen und Gruppen mit unterschiedlichen Vorstellungen von Leben, Kultur und Natur. Ein romantisiertes Bild einer früheren Agrargesellschaft bildete die Grundlage für den damaligen Naturschutz. Dies änderte sich, laut BRAND (2008), in der Weimarer Republik, in der eine zunehmend positivere Wahrnehmung der Industrialisierung mit Naturschutz zusammenfiel und die „Erhaltung eines menschlichen Erholungsraums“ im Vordergrund stand (BRAND 2008: 222), was der Beziehung von Natur- und Heimatschutz eine neue Qualität gab. KeRN (2008) hält fest, dass, im Gegensatz zu den eher pragmatischen Bestrebungen in den USA, der Naturschutz in Deutschland hauptsächlich antimodernistisch und zivilisationskritisch eingestellt gewesen sei.

Der Beginn des „Naturschutzes“ kann somit als eine Folge der zunehmenden Industrialisierung gesehen werden. Es geht konkret um Vorstellungen von Gesellschaft, die auf ein Bild von „Natur“ übertragen werden. Als „Zivilisationskritik“ kann es somit nur im Nachhinein interpretiert werden, da es sich nicht in dem Sinne um eine aktive Auseinandersetzung mit gesellschaftlichen Verhältnissen gehandelt hat, sondern um Lebensvorstellungen, die unter Rückbezug auf (abstrakte) Konzepte wie „Natur" begründet werden sollten.

Diese Entwicklungen boten einige Anknüpfungspunkte für den Nationalsozialismus, allen voran Walter Darrés „Blut und Boden Ideologie“ (MARKHAM 2004). Ab 1933 verloren ein Großteil der Natur- und Heimatschutzverbände ihre organisatorische Selbstständigkeit und wurden zum „Reichsbund Volkstum und Heimat" zusammengefasst und "gleichgeschaltet". Die ideologische Prägung des Naturschutzes durch den Nationalsozialismus machte es für die Naturschutzverbände nach dem Zweiten Weltkrieg schwer, sich wieder zu etablieren. Die Gründung des Dachverbandes „Deutscher Naturschutzring“ 1950 war ein erster Schritt, um die vereinzelten Organisationen zusammenzufassen (BRAND 2008).

In der Nachkriegszeit, nach 1945, hatten zunächst der politische und wirtschaftliche Wiederaufbau Priorität. Aufgrund der Entnazifizierungsbestrebungen der Besatzungsmächte, war eine Erlaubnis für Organisationen notwendig, um die Arbeit wieder aufnehmen zu können. Ab 1961 (in Deutschland ab 1963) nahm der WWF (World Wide Fund for Nature) eher als Förderorganisation (z.B. durch Industrielle oder PolitikerInnen) und nicht so sehr als Bewegungsorganisation, seine Arbeit auf. In der DDR erfolgte eine Art erneuter „Gleichschaltung“ und die Naturschutzverbände setzten ihre Arbeit innerhalb des Systems fort (MARKHAM 2004: 11). 
Insbesondere die Zeit des Nationalsozialismus war für die Entwicklung der Umweltbewegungen prägend. Die Instrumentalisierung von Naturvorstellungen auf der einen Seite und die Sichtweise einer „harmonischen Verbindung“ von Mensch und Natur, insbesondere mit einem agrarromantischen Charakter, finden sich bis heute (selbst in wissenschaftlichen Diskursen) in den Vorstellungen von "Umwelt" und „Ökologie“ wieder. Fraglich ist auch, wie viel von dem nationalistischen Gedankengut nach 1945 wieder in die Umweltverbände eingebracht worden ist. Im Gegensatz zu anderen Umweltverbände, die ihre Mitglieder aus der Bevölkerung bezogen, setzt sich der WWF eher aus Mitgliedern aus Politik und Wirtschaft zusammen. Der Umweltschutz wurde damit auch in diesen Kreisen zunehmend ein Thema.

\subsubsection{0er bis Anfang der 1980er Jahre}

Ab Mitte der 1960er Jahre veränderte sich die Wahrnehmung von „Naturschutz“ hin zu „Umweltproblemen“. Das romantisierende, agrarisch geprägte Bild als Gegensatz zur Industrialisierung wandelte sich nun zu einem Aufgreifen der konkreten Folgen industrieller Produktion. Das Erscheinen der Bücher „Die Grenzen des Wachstums“ des Club of Rome im Jahre 1971 und „Stummer Frühling“ von Rachel Carson 1962 prägten diese Zeit stark (BRAND 2008). Die Entstehung von Umweltbewegungen wurde zum einen durch Atombombentests und die Diskussion um radioaktive Verseuchung und zum anderen durch zunehmende Umweltkatastrophen in den 1950ern und 1960ern (darunter Tankerunglücke, Zwischenfälle in Kernkraftwerken und eine allgemeine Belastung von Wasser, Luft und Boden) zusätzlich verstärkt (KERN 2008). Parallel dazu bildeten sich viele regionale Gruppen und BürgerInneninitiativen mit einem vorrangig lokalem Fokus, die 1972 in die Gründung des BBU (Bundesverband Bürgerinitiativen Umwelt) mündeten (MARKHAm 2004). Auf politischer Ebene brachte die Regierung von Willy Brandt seit 1969 zunehmend Umweltgesetze im Bundestag ein und die erste Umweltkonferenz der Vereinten Nationen fand 1972 in Stockholm statt (BRAND 2008). Die Entwicklungen in den 1960er Jahren stellten einen bedeutenden Wendepunkt in der Geschichte des Umweltschutzes dar. KERN (2008: 105) stellt fest:

„Während sich die traditionelle Ökologiebewegung zumeist politisch neutral verhielt, den Naturschutz und die Bewahrung natürlicher Ressourcen in den Mittelpunkt stellte, thematisierte die neue Ökologiebewegung das Verhältnis zwischen Mensch und Umwelt im umfassenden Sinne und leitete daraus grundlegende politische, wirtschaftliche und soziale Forderungen ab."

Dieses Zitat spiegelt eines der großen Probleme bei der Betrachtung von Umweltbewegungen wieder. Die Unterscheidung zwischen einer „politisch neutralen“ tradi- 
tionellen Bewegung und einer neueren Bewegung ist nicht haltbar. Auch die frühere Bewegung hat versucht politische und soziale Ziele zu verwirklichen, die keinesfalls neutral waren. Der Unterschied ist eher, dass sich der Fokus auf konkretere Konfliktfelder im Sinne von „Umweltproblemen“ verlagert hat und daraus Forderungen an Politik, Wirtschaft und Gesellschaft abgeleitet wurden. Dies zeigt sich besonders darin, dass Umweltthemen vermehrt in Gesetze eingeflossen sind und in einer breiteren Öffentlichkeit diskutiert wurden.

Die Ölkrise im Herbst 1973 stellt zunächst einen Einschnitt dar: Die Regierung von Helmut Schmidt setzte auf wirtschaftliche Modernisierung, den Ausbau von Kernenergie und wirtschaftliche Probleme bestimmten die Tagesordnung. In der öffentlichen Wahrnehmung wurden Ökologie und Ökonomie zunehmend als entgegengesetzt betrachtet und es bildete sich ein Netz aus grün-alternativen basisdemokratischen Umweltgruppen (BRAND 2008). Die Proteste gegen Kernkraft und Umweltprobleme verbanden sich, mit der Folge einer verstärkten Radikalisierung von Protesten und einer höheren Polarisierung der Öffentlichkeit (MARKHAM 2004). In der DDR entstanden zunehmend Umweltgruppen aus den evangelischen Landeskirchen (BRAND 2008). Der Erfolg der Umweltbewegungen in den 1970ern in Deutschland wird, laut GoodBoDy (2002), dem relativen Wohlstand des Landes und dem Anwachsen einer gebildeten Mittelklasse zugeschrieben. Ferner bot, laut GoodBODY (2002), das Programm zur Ausweitung der Kernkraft durch die Regierung ein konkretes politisches Ziel, das studentische Bewegungen, Frauenrechtsbewegungen und Friedensbewegungen näher zusammenbrachte.

\subsubsection{0er und 1990er Jahre}

Die 1980er waren durch eine vermehrte Institutionalisierung des Umweltschutzes geprägt und Umweltthemen wurden in Politik, Wirtschaft und Gesellschaft präsenter. Die Partei Die Grünen zog 1983 ins Parlament ein. Die Regierung von Helmut Kohl verabschiedete viele neue gesetzliche Regelungen und die Debatte um das „Waldsterben 47 verstärkte den Diskurs über Umweltthemen. Das Reaktorunglück in Tschernobyl 1986 führte zu weltweit starken Protesten gegen Kernkraft und auch die SPD, als starke politische Kraft, sprach sich dagegen aus (BRAND 2008). Nach Tschernobyl bildeten sich vermehrt neue lokale Grassroot-Organisationen (Voss 2007). Richtungsweisend war der 1975 gegründete BUND (Bund für Umwelt- und Naturschutz Deutschland), der damals durch seine Verbindung von Öffentlichkeitsund Lobbyarbeit auf der einen und der Zusammenarbeit mit BürgerInneninitiativen auf der anderen Seite sehr erfolgreich wurde. 1980 wurde Greenpeace Deutschland

\footnotetext{
${ }^{7}$ Der Begriff ist insofern interessant, als dass er, im Gegensatz zum offiziellen Begriff „neuartige Waldschäden", eine emotionale Aufladung enthält. GoodBody (2002) geht davon aus, dass dies in den frühen 1980ern stark zur Verbreitung des Begriffs beigetragen hat.
} 
gegründet und erhielt daraufhin viel Zulauf. Im Gegensatz zu den anderen Verbänden agierte Greenpeace eher isoliert und wies kaum einen Bewegungscharakter auf. Der WWF hingegen wurde wegen seiner undemokratischen Strukturen von anderen Umweltverbänden kritisiert und hatte zu diesem Zeitpunkt wenig Einfluss (MARKham 2004). In der DDR erhielt der Umweltschutz zunehmend politische und gesellschaftliche Bedeutung (Gründung der „Umweltbibliothek“ in Berlin 1986 und der „Arche“ 1988) (BRAND 2008). In den letzten Monaten der DDR bekamen Umweltthemen ein sehr hohes Gewicht, da erstmals Daten zur Umweltverschmutzung zugänglich wurden, die vorher geheim gehalten wurden (BAUKLOH \& Roose 2002). In der Wirtschaft wurden in der BRD zu dieser Zeit verstärkt Umweltthemen unter dem Stichwort „ökologische Modernisierung“ aufgegriffen (BRAND 2008).

Diese Phase ist durch eine vermehrte Institutionalisierung des Umweltschutzes geprägt. Besonders die Etablierung der Grünen als Regierungspartei, hat dazu geführt, dass viele Umweltthemen gesetzlich verankert und in einer breiten Öffentlichkeit diskutiert wurden. Es zeigt sich außerdem eine stärkere Vernetzung zwischen den einzelnen Umweltorganisationen. Zu dieser Zeit hatten Umweltthemen schon ihren festen Platz in den Agenden der Parteien und wurden in den Medien diskutiert. Dies passt zur stärkeren Institutionalisierung und der weiteren Verfestigung der Verbände, die durch größere Bekanntheit mehr Zulauf bekamen und einen größeren Einfluss erhielten.

\subsubsection{Jüngste Entwicklungen}

Anfang der 1990er Jahre hatten zunächst die Wiedervereinigung Deutschlands und eine stagnierende Wirtschaft politisch eine hohe Priorität. Lokale Umweltthemen wurden in Deutschland häufig nicht mehr als dringend wahrgenommen. Dies bedeutete eine Krise und Neuorientierung für die Umweltbewegungen (MARKHAM 2004). Unter dem Stichwort „integrierter Umweltschutz" verlagerte sich der Schwerpunkt Anfang der 1990er Jahre auf ein Management von Stoffflüssen. Dieses Konzept wurde dann zusehends, insbesondere durch die UNCED-Konferenz in Rio de Janeiro, durch das der „nachhaltigen Entwicklung“ ersetzt, in der wirtschaftliche, soziale und ökologische Fragen verknüpft werden sollten (BRAND 2008). Durch die Internationalisierung wurden zudem NGOs auf internationaler Ebene immer wichtiger. Lobbyarbeit erhielt einen immer höheren Stellenwert und Umweltorganisationen schlossen sich zu transnationalen Netzwerken zusammen, bei einer zunehmenden Überscheidung zwischen Umweltbewegungen und globalisierungskritischen Bewegungen. Prägend in diesem Zusammenhang ist das Schlagwort „Umweltgerechtigkeit" geworden (KERN 2008). Zugleich wurden auf lokaler Ebene die Agenda 21-Prozesse wirk- 
sam. Durch die Gründung des IPCC 2007 ist neben der „nachhaltigen Entwicklung" der Diskurs um Klimawandel in den Vordergrund getreten, womit auch eine verstärkte Sensibilisierung der Öffentlichkeit für Klimafragen einher ging (BRAND 2008). Mittlerweile sind weniger Verschmutzungsprobleme das Thema, sondern es geht vermehrt um die „Grenzen der Belastbarkeit natürlicher Systeme“ (KeRN 2008: 108). Hinzu kommt die Debatte um das sogenannte greenwashing von Unternehmen, die versuchen ihren Gewinn durch ein „,grünes Image“ zu steigern. Für Umweltorganisationen sind eine vermehrte Professionalisierung, eine erhöhte Abhängigkeit von Spendengeldern und eine gestiegene Relevanz von Werbung zu beobachten (MARKHAM 2004).

Seit Beginn der 1990er Jahre hatten sich die Umweltbewegungen zunehmend internationalisiert. Auf der einen Seite gibt es große Umweltorganisationen, wie Greenpeace oder den WWF, die sich in ihrer Struktur verfestigen, sich professionalisieren und über feste MitarbeiterInnen verfügen. Auf der anderen Seite gibt es vermehrt kleinere Organisationen, die sich spezielleren Themen zuwenden. MARKHAM (2004) merkt an, dass Umweltprobleme in der Öffentlichkeit vermehrt als weit entfernt oder als schwer verständlich wahrgenommen werden. Das lässt sich zumindest an den Diskussionen um „nachhaltige Entwicklung“ und „Klimawandel“ festmachen. Beide Begriffe sind sehr dehnbar und in letzter Konsequenz schwer verständlich. Umweltthemen scheinen mittlerweile auf eine Weise diskutiert zu werden, die kaum überblickt werden kann und das sowohl auf wissenschaftlicher, als auch auf medialer Ebene. Dies erweckt den Eindruck, dass um Deutungshoheiten und Interpretationen dieser Begriffe gerungen wird, aber es auf der anderen Seite immer schwieriger wird, konkrete Entwicklungen zu kommunizieren.

\subsubsection{Strukturen, Strategien und Perspektiven}

Um die geschichtliche Entwicklung der Umweltbewegungen in Deutschland zu strukturieren, schlägt KERN (2008: 108-110) einige Kategorien vor, die er als „kollektive Indentitäten" der Umweltbewegungen bezeichnet:

- Traditioneller Naturschutz: Hier steht die Bewahrung oder das subjektive erleben der "Natur" im Vordergrund. Der traditionelle Naturschutz kann auch als Vorläufer der Umweltbewegungen verstanden werden.

- Lokale Umweltgruppen und BürgerInneninitiativen: Hier soll es um „lokale Lebensqualität" gehen, die gegen bauliche Eingriffe oder Umweltverschmutzung verteidigt werden soll. Die Begriffsschöpfung NIMBY („not in my backyard") ist hier ein gutes Beispiel.

- Internationale Organisationen: Hier liegt der Schwerpunkt auf globalen 
Umweltproblemen.

- Politische Ökologi£ 8 , Darunter fallen politische Parteien, wie Bündnis 90/Die Grünen.

- Ökozentrismus: Vorstellung, dass „Natur" eine intrinsischen Wert hat, der unabhängig vom Menschen ist und eine Harmonie zwischen Mensch und „Natur“ möglich und erstrebenswert ist. Darauf basieren auch viele esoterische Bewegungen.

In diese Kategorien lassen sich die meisten Kooperationsformen, die aus Umweltbewegungen hervorgegangen sind, einordnen und ähnliche Systematiken finden sich auch bei anderen AutorInnen. Dennoch können Umweltorganisationen auch in mehrere dieser Kategorien fallen.

In Bezug auf Strategien vertritt Markham (2004: 16) die folgende These: „Wenn sie ihre Zielsetzung und Strategie beschließen, agieren Umweltorganisationen immer in einem Spannungsfeld zwischen Interessengruppen- und sozialen Bewegungsstrategien. "Interessengruppenstrategien seien z.B. Lobbyarbeit, Öffentlichkeitsarbeit, maßvolle Forderungen und im Extremfall Korporatismus. Auf der anderen Seite gebe es soziale Bewegungsstrategien, die aus der Suche nach UnterstützerInnen und Spenden und dem Ausüben von Druck auf Regierungen beständen. Hierbei erscheinen häufig Zielkonflikte als Problem. Feststellen ließe sich jedenfalls, so MARKhAm (2004), dass sich die Handlungsformen und Aktionsfelder stärker ausdifferenzierten und ausdifferenzieren.

Fraglich ist, ob es sich dabei immer um ein Spannungsfeld handeln muss. Wie anfangs erwähnt, macht die Unterscheidung zwischen Interessengruppen und sozialen Bewegungen nicht in allen Fällen Sinn, aber sie hilft dabei, die Zielgruppen für Strategien auszumachen: Sind es auf der einen Seite Regierungen und die Verbindungen zu politischen und wirtschaftlichen Institutionen, so ist es auf der anderen Seite die Basis der Organisationen, also UnterstützerInnen, welche diese erst handlungsfähig machen.

Markham (2004: 15) stellt eine gewisse Konstanz in der Geschichte des Naturund Umweltschutzes fest: „Die Anziehungskraft von Natur- und Umweltschutz für die Menschen ist offenbar weder beiläufig noch zeitweilig." Laut BAUKLOH \& RoOSE (2002) fänden sich keine systematischen Studien, die eine Abnahme von Protesten, der Radikalität von Protesten oder der Zahl der Mitglieder zeigen. BLÜHDORN (2002) beobachtet eine weitere Abnahme der Radikalität von Protesten in den letzten Jahrzehnten, insbesondere durch die Aufstockung der inneren Sicherheit in

\footnotetext{
${ }^{8}$ Damit ist nicht die politische Ökologie als theoretischer Ansatz gemeint, sondern eine beschreibende Kategorie (RobBins, Hintz \& Moore 2010).
} 
Deutschland und eine Zunahme von problemspezifischen und pragmatischen Ansätzen. BAUKLOH \& RoOse (2002) geben aber zu bedenken, dass zwar die Zahl der Mitglieder hoch ist bzw. höher wird, aber sich nur eine Minderheit wirklich aktiv beteilige. Sie halten jedoch fest, dass die Schaffung eines öffentlichen Bewusstseins für Umweltbelange als einer der größten Erfolge der Umweltorganisationen angesehen werden könne. Einig sind sich die AutorInnen zumindest darin, dass nicht von einer „Krise“ der Umweltorganisationen gesprochen werden kann. Dazu gibt BLÜHDORN (2002: 120) einen Ausblick:

„Contemporary societies will, obviously, continue to deal with their redefined environmental problems - but this redefinition entails significant changes in the substance, style and ethos of environmental politics."

Ob dies „offensichtlich“ ist, sei dahingestellt, es zeigt sich jedoch, dass sich mit einer veränderten Auffassung von „Umwelt“ oder „Natur" sich die Ziele und Strategien der Umweltbewegungen verändern. Insofern haben sich die Organisationen meist den veränderten Bedingungen angepasst bzw. diese selbst beeinflusst.

\subsubsection{Ausblick}

Aus dem geschichtlichen Überblick und den Strategien und Perspektiven lassen sich einige Punkte ableiten, welche die aktuellen Entwicklungen in Umweltorganisationen und -bewegungen widerspiegeln:

- Die Komplexität der Themen nimmt zu: Zusammenhänge werden schwerer zu überschauen und Begriffe, wie „nachhaltige Entwicklung“, lassen sich fast beliebig auslegen. Dies führt vermehrt dazu, dass sich Organisationen, nur einzelnen oder spezielleren Themen widmen.

- Eine zunehmende Professionalisierung: Der Anteil an festangestellten Mitgliedern in Organisationen steigt. Das hat auch Folgen für die Struktur und Handlungsformen von Organisationen und für die Arbeit von Freiwilligen.

- Die Abhängigkeit von Spendengeldern: Für Imagekampagnen, festangestellte Mitglieder und medienwirksame Aktionsformen werden höhere Einnahmen benötigt.

- Verbindungen mit globalisierungskritischen Netzwerken: Dies kann Vor- und Nachteile haben, z.B. ergibt sich ein größerer Bekanntheitsgrad, aber dafür müssen thematische Kompromisse eingegangen werden.

- Stärkere Internationalisierung: Es wird verstärkt international zusammengearbeitet, internationale Kampagnen nehmen zu und es gibt eine breitere Themenwahl. 
- Lobbying und Korporatismus: Der Einsatz von Lobbying als Strategie nimmt zu. Dies ist verbunden mit der Gefahr abhängiger von Unternehmen oder Staaten zu werden.

- Vermehrte Institutionalisierung: Verlagerung von Umweltbewegungen hin zu einzelnen großen Umweltorganisationen.

- Aufbau von Images: Die Außendarstellung wird, insbesondere bei der Spendeneinwerbung und bei öffentlichkeitswirksamen Aktionen, immer wichtiger.

Dieser Ausblick stellt nur allgemeine Trends dar. Für die beiden untersuchten Umweltorganisationen finden sich konkretere Angaben zu Struktur, Zielen und Strategien im Abschnitt Beschreibung der untersuchten Organisationen (5.1).

\subsection{Zusammenfassung}

In diesem Kapitel geht es darum zunächst die Begriffe „Nichtregierungsorganisation“ und „soziale Bewegung“ näher zu beleuchten, um daraufhin die Entstehung und Entwicklung der Umweltbewegungen und -organisationen in Deutschland nachvollziehen zu können. Dabei wird besonders auf die Vorstellungen von „Umwelt“ und "Natur" eingegangen, um die Ziele und Vorstellungen von Umweltorganisationen besser in einen Umweltdiskurs einordnen zu können. Abschließend werden aktuelle Entwicklungen und Perspektiven für Umweltorganisationen dargestellt. Das Kapitel schildert somit den geschichtlichen Hintergrund dieser Organisationen und soll helfen, Ziele, Strategien und Naturvorstellungen, auch in Hinblick auf die Empirie, einordnen zu können. 


\section{Partizipation}

In diesem Kapitel wird der Begriff der Partizipation näher betrachtet. Zunächst wird eine Begiffsbestimmung vorgenommen, in der sich ihm über einige beispielhafte Definitionen angenähert werden soll. Darauf folgt ein Teil, der die theoretischen Hintergründe für die Auseinandersetzung mit dem Begriff liefern soll. Darin wird zunächst auf Zivilgesellschaft, als theoretischer Rahmen, dann auf zivilgesellschaftliches Enagement und Ehrenamt, eingegangen, um schließlich die individuelle Ebene von Partizipation darzustellen. Das Kapitel schließt mit einigen kritischen Bemerkungen zur Partizipation und einer eigenen Defintion des Begriffs für diese Arbeit.

\subsection{Begriffsbestimmung}

Der Begriff der Partizipation hat je nach Kontext in dem er verwendet wird eine etwas andere Bedeutung. Im Bereich der Politik und der Politikwissenschaften wird er meist mit politischer Beteiligung belegt, wie z.B. die Teilnahme an Wahlen oder lokalpolitischen Entscheidungen (BüHLMAnN 2005). Im Bereich des bürgerschaftlichen Engagements wird Partizipation als freiwillige Beteiligung im zivilgesellschaftlichen Institutionen gesehen, also der Mitarbeit in Vereinen, Verbänden, Stiftungen oder kirchlichen Gruppen (ADLOFF 2005). Verbreitet ist auch das Partizipationsverständnis aus Jürgens Habermas' Theorie des kommunikativen Handelns und das darauf aufbauende Konzept der deliberativen Demokratie. Diese sei durch Partizipation als Teil eines offenen und herrschaftsfreien Diskurses („ideale Sprechsituation“) geprägt (Kersting 2008). Der Begriff Partizipation taucht noch in anderen Zusammenhängen auf, z.B. in methodischen und theoretischen Ansätzen in der Entwicklungszusammenarbeit (CoOKE \& KothaRI 2004) oder Forschungsmethodiken (z.B. PAIN (2004) für die Sozialgeographie).

Häufig wird hervorgehoben, dass Partizipation eine besondere Bedeutung für Demokratie habe. Allerdings bleibt ein tieferes Verständnis von dem, was Partizipation genau bezeichnet, oft unklar. Abhängig von politischen Standpunkten und wissenschaftlichen Blickwinkeln wurde Partizipation unterschiedlich interpretiert: In den 1960er Jahren wurde der Begriff hauptsächlich als Mechanismus der politischen Mitbestimmung im Sinne von Wahlen oder Parteimitgliedschaften verwen- 
det. Mitte der 1970er Jahre entfernte sich der Begriff zunehmend von parlamentarischer Mitwirkung und verlagerte sich zu der Beeinflussung von parlamentarischen Prozessen. Seit den 1980ern wird er eher als alternativer Weg gesehen, politische Entscheidungen im weiteren Sinn zu beeinflussen (Bliss \& Neumann 2008).

Zunächst sollen einige Definitionen betrachtet werden, um sich dem Begriff zu nähern. Der Club of Rome (1979: 58-59, Hervorhebungen im Original) beschreibt Partizipation folgendermaßen:

„Nur wenige Worte vermögen den Anspruch der Menschen so deutlich zu machen, Entscheidungen sowohl auf lokaler als auch auf globaler Ebene, die ihre Umwelt und ihr Leben bestimmen, mit zu beeinflussen, in Verbindung mit ihrer Hoffnung auf Gleichheit und ihrer Weigerung, eine Abseitsposition oder einen untergeordneten Status zu akzeptieren. Effektive Partizipation setzt das Streben des Menschen nach Integrität und Würde voraus sowie seine Bereitschaft, die Initiative zu ergreifen. Obwohl das Recht zu partizipieren ,garantiert' werden kann, können weder die Partizipation selbst noch die damit verbundene Pflicht und Verantwortung ,gegeben' oder weggegeben werden. Echte Partizipation vollzieht sich freiwillig;"“

In diesem Zitat wird Partizipation pathetisch, wie abstrakt definiert. Es spiegelt aber sehr gut zwei Aspekte wieder, die in Definitionen immer wieder auftauchen: Zum einen gibt es einen gewissen „utopischen Anspruch“, der mit Partizipation verbunden wird. Hier wird von „Gleichheit“, „Würde“ und „mündigen“ Menschen ausgegangen, die sich für eine „bessere Welt" einsetzen. Zum anderen hat der Begriff häufig eine politisch-ideologische Färbung. Inwiefern Entscheidungen auf diese Art und Weise beeinflusst werden können, insbesonderde auf globaler Ebene, ist fraglich. Außerdem wird die „Verantwortung“ individualisiert: Alles weist darauf hin, dass Partizipation in dieser Definition von Individuen ausgeht und es sich nicht um Gruppen handelt, die diese Vision erreichen sollen. Eine Mischung aus utopischem Ideal und einer politisch motivierter Instrumentalisierbarkeit des Begriffs haben sicherlich zu seiner Popularität, aber auch zu seiner geringen Aussagekraft, beigetragen.

Eine politikwissenschaftlichere Definition bietet NAssmacher (2002: 24): Für sie ist Partizipation ,alle Tätigkeiten der Bürger zusammengefasst, die diese freiwillig mit dem Ziel unternehmen, Entscheidungen auf den verschiedenen Ebenen des politischen Systems zu beeinflussen." Auch hier geht es um die Beeinflussung von „Entscheidungen“, aber der Begriff wird restriktiver durch die Verwendung des Begriffs „Bürger“, es wird also eine bestimmte Stellung innerhalb eines demokratischen Systems vorrausgesetzt, um partizipieren zu können.

STANGE (2008 :15) kommt zu einer etwas anderen Einschätzung: „Partizipation heißt, Entscheidungen, die das eigene Leben und das Leben der Gemeinschaft betreffen, zu teilen und gemeinsam Lösungen für Probleme zu finden." In diesem 
Zitat liegt die Betonung stärker auf „Gemeinschaft“, die häufig in Verbindung mit Partizipation angesprochen wird, es aber unklar ist, was genau damit gemeint ist.

Die drei aufgeführten Definitionen stellen nur eine Auswahl dar, aber sie spiegeln unterschiedliche Aspekte und Gemeinsamkeiten wieder, die in vielen Definitionen auftauchen. Alle beeinhalten das Treffen von Entscheidungen als grundlegendes Element von Partizipation. Des Weiteren tauchen eine individuelle Ebene, sowie eine Ebene von „Gemeinschaft" auf. Außerdem wird die „Freiwilligkeit“ zu partizipieren betont. Alle Definitionen sind abstrakt gehalten und es geht nicht daraus hervor, wie sich Partizipation konkret äußert oder umsetzen lässt. Im Abschnitt Kritische Überlegungen zum Partizipationsbegriff (3.3), wird deshalb diskutiert, warum der Begriff in dieser Arbeit verwendet wird, um daraufhin eine Arbeitsdefinition zu entwickeln.

\subsection{Theoretischer Hintergrund}

Wie sich in der Begriffsbestimmung gezeigt hat, fällt es schwer, Partizipation zu definieren. Lässt man den Begriff im Raum stehen, wie es z. B. bei der Definition des Club of Rome der Fall ist, so bleibt ein Konzept, dass sehr dehnbar und unpräzise ist. Insofern ist es notwenig, eine gesellschaftstheoretische Einbettung zu finden, damit eine Arbeit mit dem Begriff Partizipation möglich wird. Die Einbettung wird in dieser Arbeit anhand des theoretischen Konzepts der Zivilgesellschaft 1 vorgenommen.

\subsubsection{Zivilgesellschaft}

Adloff (2005: 9-13) skizziert die Entwicklung des Begriffs Zivilgesellschaft: Dieser gehe ursprünglich auf Aristoteles zurück, der damit ganz allgemein das politische Gemeinwesen bezeichnet habe. Im 18. und 19. Jahrhundert sei der Begriff dann durch Autoren wie Locke, Montesquieu, Hegel oder Tocqueville stärker vom Staat und der entstehenden Marktwirtschaft abgegrenzt worden. Marx bezog ihn später auf die bürgerliche Gesellschaft der Warenproduktion. Anfang des 20. Jahrhunderts sei dann eine weiter verstärkte Abgrenzung zur Wirtschaft durch Gramsci und Deweys vorgenommen worden. Seit den 1980er Jahren werde Zivilgesellschaft im Zuge neuer sozialer Bewegungen zunehmend wieder in Deutschland diskutiert und als normatives Konzept verwendet, um die „Demokratie zu demokratisieren“ (AdLOFF 2005: 12). Seitdem sei die Verwendung des Begriffs zunehmend unübersichtlicher geworden, weil er in unterschiedlichsten wissenschaftlichen und politischen Zusammen-

\footnotetext{
${ }^{1}$ Neben Zivilgesellschaft wird auch von „Bürgergesellschaft“ gesprochen. Diese Begriffe sind nicht synnonym, aber größtenteils deckungsgleich (KOCKA 2003), so dass hier nur von Zivilgesellschaft gesprochen wird.
} 
hängen angewandt wird. In neuester Zeit werde Zivilgesellschaft in Verbindung mit der Frage nach Global Governance, einer transnationalen Zivilgesellschaft, die dem globalen Kapitalismus entgegengesetzt ist, diskutiert.

In Deutschland sei in jüngerer Zeit vermehrt zu beobachten, dass Zivilgesellschaft in Verbindung mit Arbeitsmarktpolitik, den Grenzen des Sozialstaats und politischer Steuerung genannt werde (POLlaK 2004). POLLAK (2004: 23-24) weist auf einen Grundwiderspruch in der Diskussion über Zivilgesellschaft hin: Bürgerschaftliches Engagement, als Kern der Zivilgesellschaft, sei zum einen eine notwendige Voraussetzungen für eine demokratische Gesellschaft, auf der anderen Seite soll diese aber nicht durch den Staat instrumentalisiert werden. Die Rolle des Staates in Bezug auf bürgerschaftliches Engagement und Zivilgesellschaft ist in diesem Falle widersprüchlich, der Staat schafft auf der einen Seite die notwendigen Voraussetzungen, auf der anderen Seite braucht der Staat zivilgesellschaftliche Ressourcen auf. Dies lässt sich an einem Beispiel verdeutlichen: Auf der einen Seite ist der deutsche Staat bemüht, zivilgesellschaftliche Kräfte zu stärken, indem Projekte zur Steigerung des Ehrenamtes oder des bürgerschaftlichen Engagements initiiert werden (zum Beispiel durch die Einrichtung einer Enquette-Kommission zur „Zukunft des bürgerschaftlichen Engagements" durch den Deutschen Bundestag (ENQUETTEKommission 2002) oder durch „Tage des Ehrenamtes“, die regelmäßig in verschiedenen deutschen Städten stattfinden, z.B. in Berlin (BERLIN.DE 2010)). Auf der anderen Seite werden zunehmend staatliche Leistungen eingeschränkt und auf zivilgesellschaftliche Kräfte übertragen (ROBERTs \& DEvine 2004). Insofern erscheinen einige Definitionen, wie die von SchmidT (2004: 817), als unzureichend:

„Das auf gesellschaftliche Selbstorganisation, Interessenfindung, Interessenartikulation und gemeinwohlorientierte Mitwirkung zielende und in den Bereichen zwischen Staat, Markt und Familie verankerte Netzwerk von Initiativen, Zirkeln, Vereinen, Organisationen und Arenen."

Zivilgesellschaft steht eben nicht zwischen Staat und Ökonomie, sondern es sind Bereiche, die sich gegenseitig bedingen. Es handelt sich somit nicht um einen „herrschaftsfreihen“ Raum, sondern um einen Raum, der von ökonomischen und politischen Abhängigkeitsverhältnissen durchdrungen ist (HIRSCH 2001:19). Nach HiRsch (2001) werde in den öffentlichen Diskussionen und Auseinandersetzungen erst staatliche Herrschaft legitimiert und Hegemonie erzeugt (z.B. im Sinne einer „Konsensbildung“") und zugleich sind diese Gegenstand staatlicher Eingriffe, durch die Zwänge ausgeübt werden.

Aus diesen Gründen soll Zivilgesellschaft, wie POLLAK (2004) vorschlägt, nicht als normatives Konzept, sondern als analytischer Begriff verwendet werden, durch den gesellschaftliche Prozesse, wie Partizipation, untersucht werden können. POL- 
LAK (2004: 27) liefert dazu eine Definition von civil society:

„Unter civil society sei hier die Gesamtheit der öffentlichen Assoziationen, Vereinigungen, Bewegungen und Verbände verstanden, in denen sich Bürger auf freiwilliger Basis versammeln. Diese Assoziationen befinden sich im Raum der Öffentlichkeit und stehen prinzipiell jedem offen. Die sich in ihnen engagierenden Bürger verfolgen nicht lediglich ihre persönlichen Interessen und handeln in der Regel kooperativ. Neben den bezeichneten Organisationen und Assoziationen gehört auch ungebundenes Engagement zum zivilgesellschaftlichen Bereich, sofern es sich ebenfalls durch Freiwilligkeit, Öffentlichkeit, Gemeinschaftlichkeit, sowie die Transzendierung privater Interessen auszeichnet. Formen ungebundenen zivilgesellschaftlichen Engagements sind zum Beispiel Demonstrationen, Streiks, Petitionen, Boykottmaßnahmen usw."

Diese politische Öffentlichkeit stellt keinen herrschaftsfreien Raum dar, sondern er ist durch Interessen geprägt (dies äußert sich z.B. in der „Transzendierung privater Interessen" und durch unterschiedliche Ziele). Nach PollaK (2004) sei die civil society vom Markt getrennt, weil es keine privaten Aneignungen gebe und sie von der Familie getrennt, allen zugänglich und öffentlich sei. Durch die Trennung von Markt und Staat könne aber die Zivilgesellschaft niemals die Gesellschaft als ganzes repräsentieren. PollaK (2004) klammert dabei eine „Gemeinwohlorientierung“ aus, da in zivilgesellschaftlichen Prozessen häufig andere Gruppen angeschlossen werden (z.B. BürgerInneninitiativen).

Eng in Zusammenhang mit Zivilgesellschaft steht das Konzept des sozialen Kapitals. Ausgehend von Coleman und Bourdieu hat Robert Putnam diesen Begriff in den 1990ern wieder in die Diskussion gebracht. Mit Sozialkapital bezeichne Putnam, laut AdLoff (2005), dabei Vertrauen, Normen und soziale Netzwerke, die Handlungen zu koordinieren helfen und damit Gesellschaft „erfolgreicher" machen. Die Beteiligung in Vereinen und Kooperationen generierten dabei „Kommunikations-, Kooperations- und Hilfsbereitschaft“ (AdLOFF 2005: 71-72). Auf das Konzept des sozialen Kapitals kann hier nicht näher eingegangen werden, da dies den Rahmen der Arbeit sprengen würde.

Zivilgesellschaft darf nicht als feststehendes Konzept betrachtet werden: HiRsch (2001: 20-24) hebt hervor, dass sich das Verhältnis von Staat und Gesellschaft durch Denationalisierung, Priviatisierung und die „Internationalisierung politischer Regulationskomplexe" stetig ändere. Ferner wird diskutiert, ob soziale Ungleichheit eine Voraussetzung für Zivilgesellschaft ist (NoLTE 2003). Dass Zivilgesellschaft zwischen Markt und Staat steht, wird nicht von allen AutorInnen so gesehen: Da zivilgesellschaftliche Institutionen häufig gezwungen sind, wirtschaftlich zu handeln, lassen sie sich, nach HiRsCH (2001), nicht komplett vom Markt trennen, nur weil sie nicht das primäre Interesse der Gewinnmaximierung 
verfolgten. BrunnengräBER \& WALK (2001) schließen sich dem an und definieren Zivilgesellschaft nicht als „Zwischen“ Staat und Markt sondern als komplementär zu ihnen oder in einem Wirkungszusammenhang mit ihnen stehend.

Diese Arbeit soll auf dem Zivilgesellschaftsbegriff von Pollak aufbauen, allerdings mit einigen Einschränkungen und Ergänzungen:

- Zivilgesellschaft beschränkt sich nicht nur auf den rechtlich anerkannten Status als Bürger oder Bürgerin. Es gibt Gruppen, die sich zivilgesellschaftlich betätigen und engagieren, die nicht diesen Status haben (z.B. MigrantInnen). Dass es „prinzipiell jedem offen“ stehe sich einzubringen, verdeckt bestehende Verhältnisse, die diesen Prozess besonders erschweren.

- Die „freiwillige Basis“ ist zu differenzieren: Gesellschaftlicher, politischer oder ökonomischer Druck kann dazu führen, dass sich Personen zivilgesellschaftlich einbringen. Das „freiwillige“ besteht aus der individuellen Auswahl von Alternativen sich zu engagieren.

- Es gibt keine klare Trennung der Zivilgesellschaft von Staat, Markt und Familie und es gibt Bereiche, in denen es zu Überschneidungen kommt.

- Das Konzept der Zivilgesellschaft kann politisch eingesetzt werden, um Strategien durchzusetzen oder Problemlagen zu verschleiern.

- Die analytische Komponente soll noch einmal hervogehoben werden: Es geht nicht darum, einen wünschenswerten Zustand zu definieren, sondern eine Analyse von gesellschaftlichen Verhältnissen aufzuzeigen.

- Partizipation, wie sie hier verstanden wird, findet in einem zivilgesellschaftlichen Rahmen statt.

Vor diesem theoretischen Hintergrund werden im Folgenden zwei Bereiche betrachtet, die im Zusammenhang mit gesellschaftlicher Partizipation auftauchen: Zivilgesellschaftliches Engagement und Ehrenamt.

\section{Zivilgesellschaftliches Engagement}

In Verbindung mit Zivilgesellschaft und sozialem Kapital wird häufig zivilgesellschaftliches Engagement $\left.\right|^{2}$ diskutiert, das gewissermaßen eine konkretere Umsetzung von bestimmten Vorstellungen von Zivilgesellschaft ist. Um das Konzept aufzugreifen (dies betont sogleich die politische Bedeutung des Themas), hat die Deutsche Bundesregierung 2002 eine Enquette-Kommission zur „Zukunft

\footnotetext{
2Oft tauchen auch die Begriffe „bürgerschaftliches Engagement“ oder „freiwilliges Engagement" auf, die größtenteils synonym verwendet werden.
} 
des bürgerschaftlichen Engagements", mit den folgenden Zielen, eingerichtet (ENQUETTE-Kommission 2002: 6):

„Für die Kommission ist die Kennzeichnung ,bürgerschaftlich“ verknüpft mit der Betonung von bestimmten Motiven und Wirkungen wie etwa der Verantwortung für andere, dem Lernen von Gemeinschaftsfähigkeit oder dem Aktivwerden als Mitbürger. Bürgerschaftliches Engagement bleibt nicht allein der Mitwirkung in politischen Parteien und Verbänden und der Beteiligung in Organisationen mit sozialen und politischen Zielen vorbehalten."

Es handelt sich dabei um eine relativ breit gehaltene Definition. EMBACHER \& LANG (2008: 19) fassen die Hauptaussage des Kommissionsberichts als „freiwillig, unentgeldlich und gemeinwohlorientiert" zusammen. GROBE (2006) merkt kritisch an, dass zivilgesellschaftliches Engagement dem Staat „nichts kosten“ solle und deshalb das Engagement so propagiert werde. Zu bedenken gebe es allerdings, dass nicht jedes zivilgesellschaftliche Engagement auch staatlich „wünschenswert“ sei (GROBE 2006: 28). Da sich der Kommissionsbericht auf einer eher abstrakten Ebene hält, ist nicht ersichtlich, welche Form des Engagements nun genau gefördert werden soll. Damit liegt der Verdacht nahe, dass eher Engagement gefördert wird, dass aktuellen politischen Interessen nicht zuwiderläuft. Die Kommission weist darauf hin, dass sich das bürgerschaftliche Engagement in Deutschland stark an den Bedürfnissen und Vorstellungen von erwerbstätigen deutschen Männern im mittleren Alter orientiere (ENQUeTTE-Kommission 2002), ohne das auf Vorschläge eingegangen wird, dies zu ändern. AdLOFF (2005: 126) betont, dass das Sozialkapital, darunter das bürgerschaftliche Engagement, in Deutschland „nicht über alle sozialen Gruppen gleichverteilt" sei. OfFe \& Fuchs (2001: 478) und AdLofF (2005: 127-128) beschreiben die Situation des bürgerschaftlichen Engagements in Deutschland etwas näher: Es sei 1) von der Einkommenshöhe abhängig, 2) religiöse Bindungen und 3) das Bildungsniveau hätten einen positiven Einfluss, 4) die meisten Mitglieder seien zwischen 30 und 59 Jahren alt, 5) das Engagement sei in Ostdeutschland seit der Wiedervereinigung geringer und 6) engagierten sich mehr Männer als Frauen. Es zeigt sich also, dass sich das zivilgesellschaftliche Engagement in Deutschland ungleich verteilt. Die Frage ist, welche Ursachen das hat. Denkbar wäre, das staatliche Unterstützungen nur für bestimmte Formen des Engagements gewährt werden oder die Voraussetzungen für ein Engagement verhältnismäßig hoch angesetzt sind.

Es gibt nur wenige deutschlandweite Statistiken zum zivilgesellschafltichen Engagement. In einer Studie des Bundesministeriums für Familie, Senioren, Frauen und Jugend heißt es, dass im Jahre 200470 \% aller Deutschen öffentlich aktiv waren und $36 \%$ sich freiwillig engagierten (GEnsike 2006: 9). Rosenbladt (2001: 18 und 42) 
gibt an, dass $200134 \%$ der Bevölkerung über 14 Jahre freiwillig engagiert war, davon $8 \%$ im Bereich Umwelt-, Natur- und Tierschut $2^{3}$ und $3 \%$ mit festen Ehrenämtern in diesen Bereichen. Aktuellere deutschlandweite Zahlen liegen nicht vor.

Generell, so OfFE \& Fuchs (2001), zeichne sich ein Trend zu einem eher kurzfristigen freiwilligen Engagement ab, der stärker von persönlichen Beziehungen als durch Themen geprägt und weniger bindend sei, als dies in der Vergangenheit der Fall war.

Betrachtet man zivilgesellschaftliches Engagement, so zeigen sich Gegensätze zwischen den Aussagen der Bundesregierung und den bestehenden Verhältnissen. Es wird betont, wie wichtig das Engagement sei und es zur Bürgerschaft gehöre (was auch im Widerspruch zur Freiwilligkeit stehen kann) sich einzubringen. Auf der anderen Seite ist das Engagement nicht gleichmäßig verteilt und es gibt kaum Bestrebungen daran grundsätzlich etwas zu ändern oder konkrete Maßnahmen zu ergreifen, z.B. MigrantInnen oder ärmere Menschen stärker einzubeziehen.

\section{Ehrenamt}

Neben dem zivilgesellschaftlichen Engagement, das eher gesellschaftliche und politische Aspekte betont, ist auch der Begriff des Ehrenamtes sehr verbreitet. Dieser entwickelte sich im 19. Jahrhundert in Preußen. Durch eine Verwaltungsreform sollten die kommunalen Strukturen gefestigt werden und Mitglieder des aufstrebenden Bürgertums konnten entsprechende Stellen besetzen (ENQUETTE-Kommission 2002). Diese Stellen waren mit hohem Ansehen und Prestige verbunden, daher der Begriff des „Ehrenamtes“ (GROBE 2006). Heutzutage ist dieser weit verbreitet, bezieht sich allerdings vermehrt auf stärker formalisierte, regelgebundene und langfristige Tätigkeiten (Enquette-Kommission 2002). Die Ausschreibung des „Europäischen Jahres des Ehrenamtes 2011“ unterstreicht die Bedeutung des Begriffs auch auf europäischer Ebene (EYV 2011 2010). Mitlacher \& Schulte (2005) unterstreichen die, im Vergleich zu Deutschland, größere Bedeutung des Ehrenamtes im Ausland (z.B. in Großbritannien oder den Niederlanden). Laut BRAND (2008) gehe die Bedeutung des Ehrenamtes auf lokaler Ebene in Deutschland eher zurück. In den meisten Fällen werden allerdings keine klaren Unterscheidungen zwischen den Begriffen Ehrenamt und zivilgesellschaftlichem Engagement gemacht.

\subsubsection{Partizipationstheorien}

Neben den gesellschaftstheoretischen Rahmenbedingungen gibt es einige Theorien, die das Partizipationsverhalten von einzelnen Personen beeinflussen und die in

\footnotetext{
${ }^{3}$ Die Zusammenfassung von Umwelt-, Natur- und Tierschutz in eine Kategorie ist problematisch, da es sich um sehr unterschiedliche Bereiche handelt (siehe auch KLEIN \& LÖw 2006).
} 
diesem Abschnitt dargestellt werden. Es wird sich nur auf Ansätze beschränkt, die in Hinblick auf die Empirie sinnvoll erscheinen.

Klandermans \& Oegema (1987: 519) schlagen ein Konzept für die Partizipation in sozialen Bewegungen vor, das sich auf Organisationen übertragen lässt. Demnach entwickelt sich die Partizipation in vier Schritten:

1. Die Person entwickelt Sympathie für die Bewegung/Organisation.

2. Die Person wird das Ziel von Mobilisierungsbestrebungen von Seiten der Organisation.

3. Die Person ist motiviert zu partizipieren.

4. Die Barrieren, die Partizipation verhindern, werden überschritten.

Diese Punkte werden von Oegema \& Klandermans (1994: 704) in einer späteren Arbeit noch differenziert:

"In successful mobilization campaigns, generalized action preparedness is successfully converted into the preparedness to participate in specific actions, and this specific action preparedness materializes as actual action participation."

Das Konzept beinhaltet damit zum einen die Betrebungen der Bewegungen und Organisationen, Personen einzubeziehen, und zum anderen die individuelle Motivation mitzuwirken. Allerdings gebe es, so Oegema \& Klandermans (1994), Barrieren zwischen der tatsächlichen Partizipation und der spezifischen Bereitschaft zu partizipieren. Dies könnten zum Beispiel Krankheit oder fehlende Transportmittel sein. Des Weiteren unterscheiden sie die nonconversion (Bereitschaft ist vorhanden, aber keine Partizipation, z.B. durch zu hohe Kosten, Barrieren oder zu geringe Zielansprache) und die erosion (Verlust der Bereitschaft, z.B. durch gestiegene Kosten ${ }^{4}$, Verlust der Sympathie oder Leidensdruck durch Missstände) (OEgEma \& KLANDERMAns 1994: 705-706).

Das Modell von Oegema \& Klandermans (1994) ermöglicht es, den Prozess des Einstiegs in eine soziale Bewegung bzw. Organisation genauer zu betrachten. Dabei werden verschiedene Faktoren einbezogen: die individuelle Motivation, die Mobilisierungsbestrebungen der Bewegungen bzw. Organisationen und Gründe, die Partizipation behindern.

Penner (2002) hat sich mit dem Einfluss von individuellen Neigungen und der Ausgestaltung der Organisation auf die langfristige freiwillige Mitarbeit in

\footnotetext{
${ }^{4}$ Hiermit sind nicht nur finanzielle Kosten, sondern auch immaterielle Kosten, wie zu hoher Zeitaufwand, gemeint.
} 
Organisationen beschäftigt. Dazu hat er ein konzeptionelles Modell über beginnende und andauernde freiwillige Mitarbeit entwickelt. PENnER (2002) identifiziert dafür die folgenden Faktoren: demographische Charakteristika, prosoziales Verhalten, Werte und Glaubensvorstellungen, Motive, organisatorische Faktoren und Praxen, die Verbindung zur Organisation und den situationalen Kontext. Die Gewichtung dieser Faktoren verändert sich im Laufe des Partizipationsprozesses. Hierfür gibt PenNer (2002: 461) fünf Phasen an:

1. Es besteht ein sozialer Druck, der zur freiwilligen Arbeit führt.

2. Es wird sich für die Mitarbeit entschieden.

3. Die Arbeit wird aufgenommen.

4. Es entwickelt sich eine Identität (role identity) in der Organisation.

5. Die Mitarbeit wird langfristig fortgesetzt.

Im Gegensatz zu Oegema \& Klandermans (1994), die auch die Verbindung zu Organisation betonen, fließen hier stärker die individuellen Faktoren ein. Dies sind z.B. die Werte und Glaubensvorstellungen, welche auch die Wahl der Organisation beeinflussen kann. Der situationale Kontext sollte nicht unterschätzt werden: Es hängt immer von den einzelnen Personen ab, die sich aktuell in der Organisation engagieren, ob Partizipation gelingt. Das macht es sehr komplex. PENners (2002) Ansatz betont auch, dass sich Motive und Vorstellungen über den eigenen Stand in der Organisation und über die Organisation selbst im Laufe der Zeit ändern. Wie bereits diskutiert, erscheint das Verhältnis von sozialem Druck und freiwilliger Arbeit widersprüchlich. Leider führt PENNER (2002) diesen Punkt nicht weiter aus.

Cohn, Barkan \& Halteman (2003: 314) führen drei Faktoren an, durch die sich Menschen Organisationen anschlössen: Zwang, utilitaristische Anreize oder normative Anreize, welche die Werthaltung ansprächen. Für freiwillige Tätigkeiten erscheint ihnen vorrangig der letzte Punkt relevant. Ferner gebe es mikrostrukturelle Anreize, wie Verbundenheit zur Organisation oder Freundschaft. Diese Anreize beträfen sowohl den Einstieg, als auch die langfristige Partizipation in einer Organisation. Cohn, Barkan \& Halteman (2003) kommen zu dem Schluss, dass in der Regel eine Vielzahl von Anreizen die Partizipation beeinflusse.

\subsection{Kritische Überlegungen zum Partizipationsbegriff}

Die verbreitete Verwendung des Begriffs Partizipation ist zugleich ein Problem: Unter Partizipation können sehr verschiedene Dinge verstanden werden. In politischen Kontexten oder der Entwicklungszusammenarbeit wird Partizipation häufig 
als positiv konnotiertes Schlagwort verwendet, ohne genau zu definieren, welche Aspekte des Begriffs im Vordergrund stehen. Auch in einigen wissenschaftlichen Publikationen fällt auf, dass gar nicht oder unscharf definiert wird. Abhängig von der entsprechenden Begriffsbildung kann Partizipation auch gesellschaftlichen Gruppen schaden. Roberts \& Devine (2004) weisen darauf hin, dass freiwillige Arbeit als ideologisches Instrument, im Sinne eines sense of civic responsibility genutzt wird, um Sozialabbau zu betreiben. So werten sie es z.B. als notwendige Bedingung für Public-Private-Partnerships. In diesem Sinne kann freiwilliges Engagement dazu beitragen, dass Mittel im öffentlichen Bereich gekürzt werden. Der Begriff Partizipation kann auch als Herrschaftsinstrument missbraucht werden, wie CoOKE \& Kothari (2004) für die Entwicklungszusammenarbeit in ihrem Sammelband darstellen. Partizipatives Arbeiten kann (unter Umständen) in Zusammenhängen problematisch sein, in denen ein stark systematisches Vorgehen wichtig ist (z.B. Feuerwehr oder Katastrophenschutz). Ferner können Konflikte entstehen, wenn partizipatives Arbeiten auf andere Arbeitsweisen trifft (wie z.B. hierarchisches oder delegatives Arbeiten). Wie POLLAK (2004: 35) anmerkt, engagierten sich hauptsächlich höhergebildete, vollzeitbeschäftigte Männer mittleren Alters. Geringqualifizierte, Arbeitslose und Jungendliche seien unterrepräsentiert. Damit konzentriere sich das Engagement eher auf materiell gesicherte und gesellschaftlich gut integrierte Personen. Wie bereits diskutiert, trifft dies auch auf MigrantInnen zu. KoCKA (2003: 36) spricht hier von einer „Zivilgesellschaftsfähigkeit“, die notwendig sei, bestehend aus: Zeit, Abkömmlichkeit, Deckung des Lebensunterhalts, Kommunikationsfähigkeit, Bildung und andere ungleich verteilte Ressourcen. Diese sei gesellschaftlich ungleich verteilt und beschränke die Möglichkeiten sich zivilgesellschaftlich einzubringen.

Die Möglichkeiten gesellschaftlich (besonders in einer staatlich anerkannten Weise) zu partizipieren sind also begrenzt und sozial ungleich verteilt. Dies wirkt sich auf Themen aus, die behandelt werden oder auf die Art und Weise wie gesellschaftliche Veränderungen angegangen werden. Das kann zur Reproduktion von Ungleichheit führen. Dennoch kann es durch Partizipation auch möglich sein, Alternativen $\mathrm{zu}$ den bestehenden staatlichen und wirtschaftlichen Strukturen zu entwickeln.

\subsection{Eigener Partizipationsbegriff}

Trotz der Kritik wird in dieser Arbeit von Partizipation gesprochen. Der Begriff des zivilgesellschaftlichen Engagements wird hauptsächlich in staatlichen Zusammenhängen verwendet und hat eine politische Konnotation (z.B. im Sinne eines „Werbemittels“ für Engagement). Partizipation als Begriff berücksichtigt sowohl die Mitwirkung als Teil einer Gruppe in gesellschaftlichen Prozessen (z.B. Gruppen- 
strategien), als auch die Mitwirkung von Individuen in Gruppen (z.B. hinsichtlich der Motivation oder Gruppendynamik). Um zu vermeiden, dass der Begriff losgelößt im Raum steht, wie es unter Begriffsbestimmung (3.1) kritisiert wurde, ist eine Einbettung in die Theorie der Zivilgesellschaft vorgenommen worden. Das ermöglicht es, Partizipation auch unter einem gesellschaftstheoretischen Blick reflektieren zu können.

Da es sich um eine explorative Arbeit handelt, soll der Begriff nicht zu sehr eingeschränkt werden, um die Offenheit für die Persepektiven der Interviewten offenzulassen und die Frage, wie Partizipation gelingen kann, besser beantworten zu können. Dennoch ist es für die Orientierung wichtig, eine Arbeitsdefinition aufzustellen, um den Gegenstand für die empirische Untersuchung zu bestimmen. Deshalb wird eine Arbeitsdefinition mit minimalen Kriterien, die erfüllt werden sollen, aufgestellt und weitere Aspekte benannt, die zwar einen Zusammenhang bilden können, sich aber erst in der empirischen Arbeit herauskristallisieren. Die Arbeitsdefinition für diese Arbeit lautet:

Partizipation bezeichnet die freiwillige und unbezahlte Beteiligung von Personen in Umweltorganisationen.

Unter „unbezahlt" wird verstanden, dass es für die Mitarbeit keine materiellen Zuwendungen, im Sinne einer Bezahlung, gibt. Weiterbildungsangebote oder Exkursionen können auch als eine Form von „Entlohnung“ verstanden werden, sollen in dieser Definition aber ausgeklammert werden. Mit „freiwillig“ ist gemeint, dass Personen auswählen, wofür sie ihre freie Zeit nutzen wollen und sich dann bewusst dafür entscheiden, diese Zeit bei einer bestimmten Organisation zu verbringen. Gesellschaftliche Verhältnisse, durch die Druck auf Personen ausgeübt wird (z.B. Leidensdruck aufgrund von lokalen Umweltschäden oder staatlicher Repression) und die sich daraufhin in zivilgesellschaftlichen Bereichen einbringen, werden in dieser Definition nicht berücksichtigt.

Die Arbeitsdefinition soll durch die folgenden Punkte ergänzt werden, auf die besonderes Augenmerk in der Auswertung des empirischen Materials gelegt werden soll:

in Gruppen Partizipation findet in Gruppen stat $t^{5}$

aktiv Partizipation geht über die reine Anwesenheit in Gruppen oder Organisationen hinaus, ein gewisser Grad an Eigeninitiative ist erforderlich, um zu partizipieren.

\footnotetext{
${ }^{5}$ Wie bereits angemerkt, wird die politische Partizipation, wie z.B. die Teilnahme an Wahlen, hier nicht behandelt.
} 
interessengeleitet Die Beteiligung verfolgt einen oder mehrere Zwecke. Dies können z.B. das Erreichen von gesellschaftlicher Veränderung, Spaß oder die Qualifikation für den Arbeitsmarkt sein. Ziele können auch unterbewusst vorhanden sein. Partizipation ist somit ein ständiger Abgleich von verschiendenen Interessen.

motiviert Personen haben eine Motivation sich einzubringen. Dies ist Voraussetzung für langfristige Partizipation.

Lernprozess Partizipation ist ein Lernprozess.

Entscheidungsprozess Partizipation beinhaltet die Beteiligung an Entscheidungen.

\subsection{Zusammenfassung}

Zunächst geht es darum, den Begriff der Partizipation gesellschaftlich einzuordnen und sich ihm über Definitionen anzunähern. Danach erfolgt eine gesellschaftstheoretische Einbettung durch das Konzept der Zivilgesellschaft und die Begriffe zivilgesellschaftliches Engagement und Ehrenamt werden diskutiert. Daraufhin werden individualisiertere Erklärungsansätze zur Partizipation vorgestellt. Das Kapitel schließt mit einer kritischen Diskussion des Partizipationsbegriffs, einer Erläuterung, warum das Konzept verwendet wird und einer Arbeitsdefinition von Partizipation. 


\section{Forschungsdesign und Methodik}

In diesem Kapitel wird das Forschungsdesign und die darin verwendete Methodik vorgestellt. Da sich qualitative Forschung an bestimmten theoretischen Positionen und Prämissen orientiert, werden diese zunächst dargestellt. Der Abschnitt über das Forschungsdesign beschreibt dann, was und auf welche Weise in dieser Arbeit untersucht wird, einschließlich der Ziele, der Fragestellung, Fallauswahl und Erhebungsmethode. Das Kapitel schließt mit einer Beschreibung des Transkriptions-, Aufbereitungs- und Auswerteverfahrens der Interviews.

\subsection{Theoretische Perspektive und Prämissen}

Hier soll zweierlei diskutiert werden: Zum einen wird auf die Vorteile und Grenzen qualitativer Forschung eingegangen, die wichtig für die spätere Reflexion des Verfahren sind. Zum anderen werden die Bedeutung von Hypothesen und die Anwendung von Gütekriterien für die qualitative Forschung diskutiert.

\subsubsection{Vorteile qualitativer Forschung}

Wie im Teil Forschungsstand (1.2) beschrieben, liegen zum Thema hauptsächlich quantitative Erhebungen vor. Hier soll ein anderer Ansatz verfolgt werden: Um die individuellen Sichtweisen und Wahrnehmungen zur Partizipation erfassen zu können, bietet es sich an, die Befragten selbst zu Wort kommen zu lassen. Bei LAMNEK (1995: 171) finden sich einige Punkte, die für die Anwendung von qualitativer Forschung sprechen:

„In der Datenerhebung sind die qualitativen Methoden in der Regel valider, weil

- die Daten näher am sozialen Feld entstehen,

- die Informationen nicht durch Forscherraster prädeterminiert sind,

- die Daten realitätsgerechter und angemessener sind,

- die Relevanzsysteme der Untersuchten berücksichtigt werden,

- die Methoden offener und flexibler sind,

- die kommunikative Verständigungsbasis existiert, 
- eine sukzessive Erweiterung der Untersuchungsbasis auch auf extreme Fälle möglich ist."

Qualitative Forschung kann damit als „näher am Gegenstand“ bezeichnet werden, weil sie versucht eine verstehende Perspektive einzunehmen, ohne vorschnell zu generalisieren. Im Vergleich zu quantitativen Verfahren steht also eine andere Perspektive im Vordergrund: Soziale Phänomene sollen möglichst unvoreingenommen ${ }^{1}$ und umfassend untersucht werden. Dies ist natürlich nur annähernd möglich.

\subsubsection{Aufstellen von Hypothesen}

Die Formulierung von Hypothesen stellt sich in der qualitativen Forschung im Gegensatz zu einer quantitativen Herangehensweise anders dar: Hier stehen die Angemessenheit der gewählten Methodik und die Offenheit gegenüber Neuem im Vordergrund. Es gibt unterschiedliche theoretische Lager, die von einem völligen Verzicht auf sogenannte Ex-ante-Hypothesen, bis hin zu fest vorgegebenen Hypothesen reichen. Einen Ansatz, der sich dazwischen bewegt, liefert MEINEFELD (2008: 272, Hervorhebungen im Original):

„Wenn wir lernen, zwischen der prinzipiellen methodischen Offenheit und der Expliziertheit, mit der das Vorwissen reflektiert und ausformuliert wird, zu unterscheiden, wird es möglich, die Formulierung von Hypothesen mit dem Rekonstruieren gegenstandsspezifischer Bedeutungsgehalte zu vereinbaren. Die Offenheit für das Neue hängt gerade nicht davon ab, dass wir auf der inhaltlichen Ebene das Alte und Bekannte nicht bewusst gemacht haben, sondern, davon, in welcher Weise wir die Suche nach dem Neuen methodisch gestalten."

Hier zeigen sich zwei wichtige Grundlagen qualitativen Arbeitens: zum einen die Offenheit gegenüber dem Gegenstand und zum anderen die Reflexion der Arbeitsschritte. Auf diese beiden Punkte soll auch in dieser Arbeit Wert gelegt werden. Insofern finden sich in dem Abschnitt Forschungsdesign (4.2.2) Hypothesen, die vor der Interviewtätigkeit aufgestellt wurden. In der Auswertung soll allerdings explizit darauf geachtet werden, ob diese Hypothesen gegenstandsangemessen sind und ob sie einer Erweiterung bedürfen. Hypothesen enthalten Vorannahmen und Vorwissen, die es zu reflektieren gilt. Somit geht es nicht nur darum Hypothesen zu bestätigen oder abzulehnen, sondern auch die Entstehungsbedingungen der Hypothesen zu untersuchen, um den Forschungsprozess einschätzen zu können und damit mehr über

\footnotetext{
${ }^{1}$ Unvoreingenommenheit ist im Grunde nicht möglich, da Menschen eine bestimmte Perspektive auf die Welt haben, die zum Beispiel durch Erziehung, Werte u.ä. bestimmt ist. Anspruch der qualitativen Forschung solle es aber sein, diese Voreingenommenheit im Forschungsprozess zu reflektieren und als Teil der Forschung zu begreifen.
} 
den Untersuchungsgegenstand zu erfahren. Diese Perspektive vertritt auch LANMEK (2005) indem er betont, dass es einer der konstitutiven Momente qualitativer Forschung sei, durch den Abgleich von theoretischem Vorverständnis und qualitativ erhobenen Daten, immer wieder zu neuen Präzisierungen, Modifizierungen und Revisionen von Theorien und Hypothesen zu kommen. Im letzten Kapitel (7) soll dann im Nachhinein die Praxis der Hypothesenbildung über den gesamten Forschungsprozess reflektiert werden.

\subsubsection{Gütekriterien}

Ausgang der qualitativen Forschung ist eine Kritik an einem objektivistischem Wissenschaftsverständnis. HELFFERICH (2005: 138) betont den „angemessenen Umgang mit Subjektivität", der für qualitative Forschung zentral sei. Interviews seien immer kontextabhängig und eine Stärke sei die Einbeziehung der Produktion von Versionen von Erzähltem in bestimmten Kontexten und nicht nur die Produkte. Dies zeigt, dass sich qualitatives Forschen nicht an den herkömmlichen Kriterien, wie denen quantitativer Verfahren messen lassen. Allerdings würde ein Verzicht auf Kriterien zu einer Willkürlichkeit und Beliebigkeit im Forschungsverlauf führen, die problematisch für die Anerkennung qualitativer Forschung sei (STEINke 2008). Damit stellt sich die Frage, inwiefern Erkenntnisse begründet oder verallgemeinert werden können. HelfFERICH (2005: 139-140) schlägt dafür drei Momente vor, anhand derer der Forschungsverlauf kontrolliert werden kann:

- Methodische Kontrolle durch Offenheit: Schaffen von Erzählraum und kritisches Überprüfen von aufgestellten Hypothesen.

- Methodische Kontrolle über Reflexivität: Bewusstmachen und explizieren des Kontextes und die reflexive Einbeziehung der Kommunikation im Forschungsprozess.

- Methodische Kontrolle über intersubjektive Nachvollziehbarkeit: Festlegen von Interviewregeln und Kodierverfahren, sowie die Dokumentation des Forschungsverlaufs.

Diese spiegeln auch die Vorteile qualitativen Arbeitens wieder (siehe 4.1.1) und ermöglichen es, den Forschungsverlauf nachvollziehbar zu gestalten. Auf diese Kriterien soll im letzten Kapitel (7) nochmals eingegangen werden, um sicherzustellen, dass sich die Empirie dieser Arbeit an den Qualitätsmerkmalen qualitativer Forschung messen kann. HelfFerich (2005) betont, dass Fehler, die sich rekonstruieren ließen, auch zu neuen Erkenntnissen führen können. 


\subsection{Forschungsdesign}

Unter Forschungsdesign wird, nach FLICK (2007), die Planung einer Untersuchung, die Datenerhebung und Analyse, sowie die Auswahl der zu berücksichtigten Fälle verstanden.

\subsubsection{Ziele der Untersuchung}

Es gibt verschiedene Ziele, die mit dieser Arbeit verfolgt werden sollen:

- Es soll ein Verständnis dafür entwickelt werden, wie Partizipation in umweltpolitischen Kontexten abläuft. Dabei konzentriert sich diese Arbeit auf die individuellen Sichtweisen der TeilnehmerInnen und ihre Motivation, die Form der Zusammenarbeit, ihre Ziele und ihre Vorstellungen über Partizipation.

- Durch einen Vergleich zwischen zwei verschiedenen Umweltorganisation, sollen die Sichtweisen auf Partizipation herausgearbeitet werden, um Unterschiede und Gemeinsamkeiten aufzuzeigen. Außerdem ist es möglich durch die Einbeziehung von Organisationen mit möglichst unterschiedlichen Strukturen einen umfassenderen Eindruck vom Themenfeld zu erlangen, weil ein breiteres Spektrum an Fällen berücksichtigt wird.

- Eine qualitative vergleichende Studie ermöglicht es Faktoren festzustellen, die das Partizipationsverhalten beeinflussen, die durch rein quantitative Studien nicht festzustellen sind. In diesem Feld gibt es kaum qualitative Studien, so dass diese Arbeit zu einer umfassenderen Betrachtung des Forschungsfeldes beiträgt (vergleiche auch Forschungsstand (1.2)).

- Durch diese qualitative Untersuchung können neue Forschungsfelder zur Partizipation entdeckt werden, die durch andere qualitative Methoden (z.B. biographische Interviews) oder quantitative Methoden (z.B. standardisierte Fragebögen) ergänzt werden können.

Die Ziele werden am Ende der Arbeit unter Reflexion und Fazit (7) in Zusammenhang mit den Ergebnissen der Untersuchung diskutiert.

\subsubsection{Fragestellung und Hypothesen}

Grundlegend geht es in dieser Arbeit um die Untersuchung von Partizipation in zwei verschiedenen Umweltorganisation. Die zentrale Fragestellung lautet somit:

Wie gestaltet sich Partizipation in den beiden Umweltorganisationen, DJN und Greenpeace in Göttingen, und gibt es Gemeinsamkeiten und Unterschiede zwischen ihnen? 
Ausgehend von der Forschungsfrage wurden Leitfragen gebildet, die als „roter Faden“ für die Untersuchung dienen und die Interviews strukturieren. Die folgenden Leitfragen ergaben sich dabei für die Untersuchung:

- Warum beteiligen sich Menschen in Umweltorganisationen?

- Wie ist die Motivation, die zur Mitarbeit in diesen Organisationen geführt hat?

- Wie wird zusammengearbeitet?

- Was wird unter Partizipation verstanden?

- Welche Auswirkungen hat das Engagement für die Partizipierenden und für andere?

- Wie sind die Organisationen aufgebaut?

- Wie lassen sich die Organisationen in das weitere Feld der deutschen Umweltbewegung einordnen?

- Wie unterscheiden sich die beiden Organisationen in den oben genannten Punkten?

- Wie lässt sich eine bessere Beteiligung erreichen?

Diese Leitfragen spezifizieren Aspekte, die für die Beantwortung der Forschungsfrage wichtig sind. Auf der Forschungsfrage basierend, wurden des Weiteren Hypothesen gebildet, die im Laufe der Arbeit untersucht werden sollen. Wie unter Aufstellen von Hypothesen (4.1.2) angemerkt stehen diese Hypothesen nicht für sich, sondern sie beinhalten bestimmte Vorannahmen, die am Ende der Arbeit (7) reflektiert werden sollen. Forschungsfrage, Leitfragen und Hypothesen beruhen auf dem Hintergrund der Arbeitsdefinition von Partizipation (3.4). Die Hypothesen helfen bei der Beantwortung der Forschungsfrage, wohingegen durch die Leitfragen der explorative Charakter der Arbeit betont werden soll und um den Blick im Vorhinein nicht zu stark einzuschränken.

\section{Es bestehen Unterschiede im Verständnis von Partizipation und deren Umsetzung zwischen den beiden Organisationen.}

Diese Hyptothese soll dabei helfen, die Vorstellungen von Partizipation in den Vordergrund zu rücken, um herauszufinden, ob damit Aussagen über die Umweltorganisationen und deren Arbeitsweise getroffen werden können. 


\section{Die Faktoren, welche die Partizipation direkt beeinflussen, sind in beiden Organisationen ähnlich.}

Es gibt Aspekte, welche die Partizipation generell beeinflussen und bei denen es keine wesentlichen Unterschiede zwischen den beiden Organisationen gibt. Diese können möglicherweise generelle Vorraussetzung für freiwillige Arbeit oder Partizipation sein.

\section{Der DJN ist in seinen Aktivitäten eher nach innen gerichtet, Greenpeace eher nach außen.}

Der Schwerpunt der Arbeit der beiden Organisationen unterscheidet sich: Der DJN legt mehr Wert auf die individuelle Entwicklung der eigenen Mitglieder, wohingegen Greenpeace stärker darauf setzt, gesellschaftliche oder politische Veränderungen durch Außenwirkung zu erreichen. Hier interessiert besonders die individuelle Interpretation der Interviewten.

\subsubsection{Fallauswahl}

Es wurden InterviewparterInnen aus zwei unterschiedlichen Umweltorganisationen befragt. Bei den Organisationen handelt es sich um Greenpeace und den Deutschen Jugendbund für Naturbeobachtung (DJN). Bei der Auswahl der Organisationen wurde darauf Wert gelegt, dass sie einen möglichst unterschiedlichen strukturellen Aufbau haben und über unterschiedliche Formen der Beteiligung verfügen. Aus den jeweiligen Organisationen wurden dann Personen befragt. Es folgt eine Beschreibung der Auswahl der InterviewpartnerInnen: Int1 und Int6 konnten über Bekannte vermittelt werden, die selbst lange Zeit im DJN aktiv waren. Int2 ergab sich als Empfehlung des Interviewpartners aus Int1. Die InterviewpartnerInnen aus Int3 und Int4 ergaben sich auf Nachfrage während eines Greenpeace-Plenumstreffens. Der Kontakt für Int5 ergab sich auch dort, wurde aber erst später hergestellt. Für die Interviews wurden Codes (Int1 bis Int6) vergeben, um die Anonymität der Interviewten zu gewährleisten. Mit der Anzahl von sechs Interviews wurde versucht, einen Kompromiss zwischen einer der Fragestellung angemessenen Repräsentativität und einem, für eine sechsmonatige Arbeit vertretbaren, Auswertungsaufwand zu finden. Ein Überblick über die interviewten Personen findet sich im Anhang A.2.

\subsubsection{Erhebungsmethode}

\section{Qualitative Interviews}

DunN (2000) gibt als Stärken eines Interviews an, dass es die Möglichkeit gibt Wissenslücken zu füllen, komplexes Verhalten und Motivationen zu erforschen, Ein- 
blicke in eine Vielzahl von Meinungen und Erfahrungen zu bekommen und den InformantInnen auf respektvoller und wertschätzender Ebene zu begegnen. Um diese Vorteile nutzen zu können, kamen bei der Erhebung offene, halbstrukturierte Interviews mit narrativen Elementen zum Einsatz, die sich an HELFFERICH (2005) orientierten. Um die Fragestellung beantworten zu können, erscheint es wichtig, zum einen möglichst offene Fragen zu stellen, um den persönlichen Einschätzungen und Erfahrungen der Interviewten gerecht zu werden. Zum anderen sollte eine Struktur gewährleistet sein, um später Kategorien bilden zu können und einen Vergleich der Interviews zu ermöglichen.

\section{Interviewleitfaden}

In den Interviewleitfaden sind Aspekte der Ziele der Arbeit, der theoretischen Hintergründe und der Fragestellung eingeflossen. Der Leitfaden findet sich im Anhang dieser Arbeit A.1 Bei der Erstellung des Leitfadens wurden die Anmerkungen zur Fragenauswahl und Reihenfolge von HELFFERICH (2005) berücksichtigt und einige Fragen sind an den Leitfaden von Moser (2010) angelehnt. Der Interviewleitfaden gliedert sich grob in die Teilbereiche:

- Einstieg in die Gruppe und Formen der Zusammenarbeit

- Motivationen für die Mitwirkung

- Verständnis von Partizipation

- Bedeutung von Natur- und Umweltschutz

- Abschlussfragen

Je nach Interviewsituation ist die Abfolge der Fragen oder die Formulierungen leicht verändert worden, allerdings wurde Wert darauf gelegt, die Bedeutung der Frage zwischen den Interviews beizubehalten, um einen späteren Vergleich zu ermöglichen. Nach Int2 wurden einige der Fragen etwas umformuliert, um die Verständlichkeit zu erhöhen.

\section{Ablauf der Interviews}

Zu Beginn der Interviews wurden die Befragten darauf hingewiesen, dass die Namen und Daten anonymisiert werden, um dem Schutz der Interviewten gerecht zu werden (angelehnt an Hermanns 2008). Außerdem wurde darum gebeten, ein digitales Aufnahmegerät verwenden zu dürfen. Vor Interviewbeginn wurde betont, dass jede Aussage für die Auswertung relevant sei, um die Offenheit der Interviews zu unterstützen. Ziel war es, den Erzählfluss der Befragten anzuregen. Deshalb sollte der 
Leitfaden nur zur Orientierung dienen, um eventuelle Nachfragen stellen zu können. Die Interviewten haben den Leitfaden während der Interviews nicht eingesehen. Die einzelnen Interviews hatten eine Dauer von etwa 30 bis 120 Minuten.

\subsubsection{Aufbereitungsverfahren}

Bei der Auswertung sollte die inhaltlich-thematische Ebene im Vordergrund stehen. Dies wurde auch bei der Auswahl eines Transkriptionsverfahrens berücksichtigt, um auf der einen Seite der Fragestellung gerecht zu werden und auf der anderen Seite den Transkriptionsaufwand zu reduzieren. Die transkribierten Interviews sind auf einer CD im Anhang dieser Arbeit zu finden. In Anlehnung an Mayring (2002, 2007) wurde eine wörtliche Transkription mit teilweiser Übertragung ins Schriftdeutsch mit den folgenden Regeln gewählt:

- Es wurde größtenteils wörtlich transkribiert. Unvollständigkeiten und Wiederholungen wurden teilweise weggelassen.

- Der Inhalt sollte im Vordergrund stehen, insofern wurden Wörter wie „äh“ weggelassen. Dialektfärbungen wurden eingedeutscht, echte Dialekte belassen.

- Unverständliche Stellen wurden mit [unverst.] versehen, teilweise mit Zeit- oder Wortangabe.

- Annonymisierte Stellen wurden mit $[\mathrm{xxx}]$ gekennzeichnet.

- Bei Pausen oder Stockungen im Gespräch wurden „... “verwendet. Die Anzahl der Punkte gibt eine Einschätzung der Pausenlänge.

- Auffälligkeiten, wie zum Beispiel Lachen, wurden in eckigen Klammern angemerkt.

- Es gelten die Abkürzungen I. für Interviewer und B. für Befragte bzw. Befragter.

Die Genauigkeit der Transkription soll sich an dem Auswertungsverfahren orientieren (FLICK 2008). Da hier eine qualitative Inhaltsanalyse durchgeführt wurde, erscheint das Aufbereitungsverfahren als angemessen.

\subsubsection{Auswertungsverfahren}

Als Auswertungsverfahren wird die von MAYRING (2007) beschriebene qualitative Inhaltsanalyse angewandt. Dabei handelt es sich um systematische Textanalyseverfahren, welche 
„die Stärken der kommunikationswissenschaftlichen Inhaltsanalyse (Theoriegeleitetheit, Regelgeleitetheit, Kommunikationsmodell, Kategorienorientiertheit, Gütekriterien) nutzen, um qualitative Analyseschritte (induktive Kategorienentwicklung, Zusammenfassung, Kontextanalyse, deduktive Kategorienanwendung) methodisch kontrolliert vollziehen zu können." (MAYRING 2000: 8)

Neben der Untersuchung der inhaltlichen Ebene sind damit auch Schlüsse auf "latente Sinngehalte“ in den Texten möglich (MAYring 2008). Für die Fragestellung stellt die qualitative Inhaltsanalyse nach den Kriterien von MAYRING $(2002,2007)$ eine adäquate Methode dar, da es sich nicht um eine rein explorative Arbeit handelt und sie zu dem Erhebungs- und Aufbereitungsverfahren der Daten passt. Konkret wird dabei die Zusammenfassung als inhaltsanalytische Methode verwendet, um das Material systematisch zu reduzieren und einen überschaubaren Corpus zu erhalten, der sich nicht zu weit vom Ausgangsmaterial entfernt (MAYRING 2007). Die einzelnen Auswertungsschritte sind zum Überblick in der Abb. 4.1 dargestellt. 


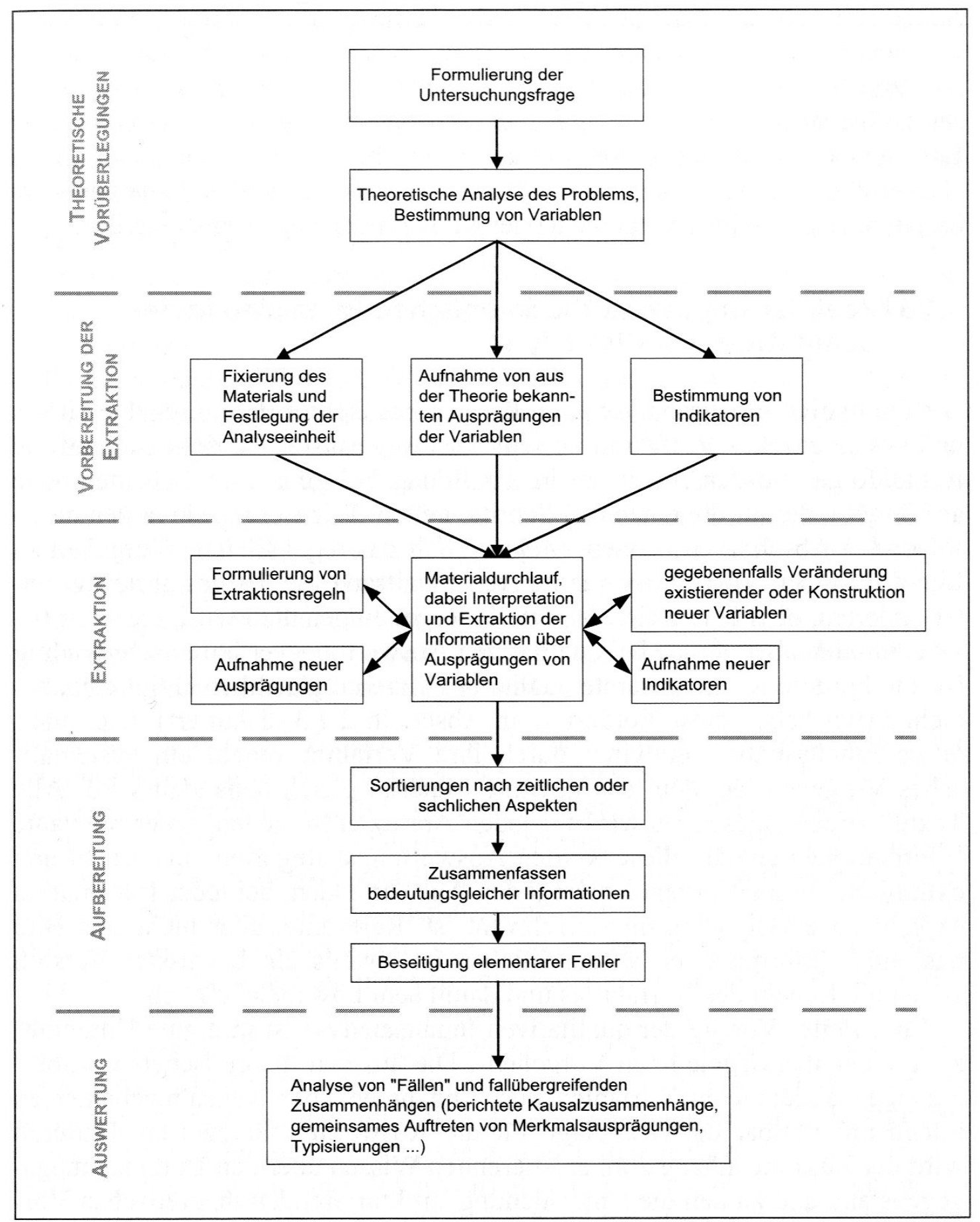

Abbildung 4.1: Ablauf einer qualitativen Inhaltsanalyse (GLÄSER \& LAUDEL 2009: 203) 
Aus den theoretischen Vorüberlegungen und den Interviews wurden dann die Kodierkategorien gebildet. Zu den Kodierkategorien gibt es noch entsprechende Leitfragen, um die Aussagen der Interviews leichter zuordnen zu können. Die folgenden Kategorien wurden verwendet:

- Motivation

- Welche Rollen spielen Umwelt- und Naturschutz für die Motivation?

- Welche Rolle spielt die Gruppe/Zusammenarbeit für die Motivation?

- Was hat die Wahl der Organisation beeinflusst?

- Was sorgt für anhaltende Motivation?

- Engagement

- Was haben die Interviewten selbst von ihrem Engagement?

- Was haben andere vom Engagement der Interviewten?

- Partizipation

- Was heißt Partizipation?

- (Wie) Kann sie erlernt werden?

- Welche Probleme gibt es mit Partizipation?

- Zusammenarbeit

- Wie werden Entscheidungen getroffen?

- Wie werden Themen ausgewählt?

- Wie werden Projekte umgesetzt?

- Welche Rolle spielt das Alter?

- Struktur

- Wieviele Mitglieder gibt es?

- Wie sind die Gruppen strukturiert?

- Wie sieht die übergeordnete/überregionale Organisation aus?

- Welche Aktivitäten und Aufgaben gibt es?

- Welche Eigenschaften werden den Organisationen zugeschrieben?

- Natur und Umwelt

- Welche Aussagen werden zu Natur- und Umweltschutz getroffen?

- Wie wird das Verhalten von anderen in Bezug auf Umwelt- und Naturschutz eingeschätzt? 
- Kommunikation

- Wie verläuft die Kommunikation über das Internet?

- Wie verläuft die Kommunikation mit (externen) Gruppen?

- Selbsteinschätzung und Werdegang

- Wie schätzen sich die Befragten selbst im Verhältnis zur Organisation ein?

- Wie ist der Werdegang der Befragten?

- Sonstiges

Bei der Auswertung wurde sich an GLÄSER \& LAUDEL (2009) orientiert. Um das Verfahren der Textextraktion zu verdeutlichen, folgt ein Auswertungsbeispiel aus den aufgenommenen Daten:

\section{Auswertungsbeispiel}

Der erste Schritt besteht aus einer Paraphrasierung der Originalzitate aus den Interviews. Die soll anhand des folgenden Zitats verdeutlicht werden:

„Ja, also gute Erfahrungen haben wir dann gemacht, wenn dann jemand sich bereit erklärt hat, so ein bisschen sich den Hut aufzusetzen für eine Aktion, dann ist es eigentlich ganz gut gelaufen, weil man dann einen Ansprechpartner gehabt habt" (Int3: 137-139) ${ }^{2}$

Aus dem Zitat wird die Paraphrase: „gute Erfahrung, wenn jemand die Verantwortung für Aktionen übernimmt und Ansprechpartner ist" gebildet. Daraus ergab sich dann die folgende Generalisierung aus der Aussage: „positiv, wenn es Verantwortliche gibt“. Die Generalisierungen werden gesammelt und Wiederholungen und nicht zentral inhaltstragende Phrasen gestrichen. Daran schließt sich eine Zusammenfassung der Generalisierungen mit ähnlichen Aussagen an. Die Aussage im Beispiel ist dann der Kategorie „Zusammenarbeit“ in der Unterkategorie „Umsetzung von Projekten“ zugeordnet worden.

Die Unterkategorie „Umsetzung von Projekten“ setzt sich dabei aus verschiedenen Aussagen zusammen. Diese sind beispielsweise:

- unerledigte Aufgaben, wenn niemand verantwortlich

- manchmal fehlende AnsprechpartnerInnen für andere Gruppen

\footnotetext{
${ }^{2}$ Passagen aus den Interviews werden mit der Interviewnummer (Int1 bis Int6) und den jeweiligen Zeilenangaben zitiert. Die Transpkripte der Interviews finden sich auf einer CD im Anhang dieser Arbeit.
} 
- kurzfristige Aktionen gelingen immer

- chaotisch ohne HauptansprechpartnerInnen

- Gruppenzusammensetzung bestimmt über Erfolg

Mit Hilfe der Generalisierungen und der Kategorien und Unterkategorien können die Daten somit verdichtet werden. Diese Form der Auswertung bildet dann die Grundlage für die Darstellungen unter Empirie (5).

\subsection{Zusammenfassung}

Ziel diese Kapitels ist es zunächst, die zu Grunde gelegten methodologischen und theoretischen Hintergründe für die qualitative Forschung darzustellen. Im darauf folgenden Abschnitt wird auf das Forschungsdesign eingegangen, in dem die Fragestellung, die Ziele der Untersuchung, die Auswahl der Fälle, die Erhebungsmethode und das Aufbereitungs- und Auswertungsverfahren dargestellt werden. Über leitfadengestützte Interviews, die anschließend mit einer zusammenfassenden qualitativen Inhaltsanalyse ausgewertet werden, wird der Forschungsfrage nachgegangen, wie Partizipation in zwei Göttinger Umweltorganisationen abläuft und wie sie sich unterscheidet. Dieses Kapitel ist somit wichtig, um den qualitativen Forschungsprozess dieser Arbeit transparent zu machen und am Ende der Arbeit Rückschlüsse auf die Güte der Ergebnisse und ihrer Generalisierbarkeit machen zu können. 


\section{Empirie}

In diesem Kapitel werden die empirischen Ergebnisse dargestellt. Zunächst werden die beiden untersuchten Umweltorganisationen vorgestellt. Darauf folgt dann die Auswertung der Interviews.

\subsection{Beschreibung der untersuchten Organisationen}

Wie unter Fallauswahl 4.2.3 dargestellt, wurden zwei Umweltorganisationen ausgewählt, die in ihrer Struktur, in ihren Zielen und in ihren Strategien sehr unterschiedlich sind. In diesem Abschnitt soll zuerst ein kurzer Überblick über die beiden Organisationen gegeben werden, um die Aussagen der InterviewpartnerInnen in einen größeren institutionellen Zusammenhang einordnen zu können.

\subsubsection{Greenpeace}

Es gibt verhältnismäßig viele Veröffentlichungen zu Greenpeace, sowohl zu Greenpeace Deutschland, als auch zu Greenpeace International. Darunter werden meist bestimmte Aspekte hervorgehoben, wie zum Beispiel die Öffentlichkeitsarbeit (Voss 2007) oder die Geschichte der Organisation (MARKham 2004). Hier soll nur ein kurzer Überblick erfolgen, da umfangreiche Darstellungen anderweitig vorliegen.

Zusammen mit dem BUND, dem WWF und dem NABU ist Greenpeace eine der großen vier Umweltorganisationen in Deutschland. Als Reaktion auf Atomwaffentest in den Vereinigten Staaten, wurde Greenpeace im Jahre 1971 gegründet, 1980 folgte Greenpeace Deutschland (Voss 2007). Durch die hohe mediale Aufmerksamkeit auch im internationalen Bereich und durch das wachsende Interesse an Umweltfragen durch die Bevölkerung entwickelte sich Greenpeace Deutschland, durch ein großes Wachstum an UnterstützerInnen, Finanzierung und bezahlten MitarbeiterInnen, zu der größten und finanzstärksten Umweltorganisation in Deutschland (MARKHAM 2008)

\section{Strategien}

Greenpeace setzt mehr als andere Organisationen auf öffentlichkeitswirksame und spektakuläre Aktionen, um auf Natur- und Umweltprobleme aufmerksam 
zu machen. Sie setzen damit eine „bewusst symbolische Politik“ ein, um ihre Ziele zu erreichen (Voss 2007: 140). Viele Aktionen sind dabei professionell geplante Medienereignisse, die darauf ausgelegt sind, das öffentliche Bewusstsein für Umweltfragen zu steigern und Regierungen und Unternehmen unter Druck zu setzen. Dabei liegt ein Schwerpunkt auf Problemen, die in der Öffentlichkeit leicht zu vermitteln sind. Greenpeace kann diese Strategie nutzen, da er über gute Kontakte zur Presse verfügt und er ist darauf bedacht Informationsmaterial zu Umweltproblemen oder zu eigenen Aktionen der Presse und Öffentlichkeit zur Verfügung zu stellen (MARKham 2008). Für die Erreichung ihrer Ziele setzt Greenpeace auf Kampagnenarbeit. Diese Kampagnen werden für die Dauer von einigen Monaten angelegt, sind sehr strikt und konfrontativ geplant und zielen meist auf einen bestimmten GegnerInnen aht (WALlRafF 2010). Diese Form der Einflussnahme auf politische Entscheidungen und die Medienwirksamkeit der Aktionen tragen sicherlich zum großen Bekanntheitsgrad von Greenpeace bei. Laut WALlRAFF (2010) gebe es vermehrt Bestrebungen, Projekte umzusetzen, die nicht auf Konfrontation setzen, wie die Entwicklung des Solarkühlschranks „SolarChill“ oder der Einsatz für Filter in Dieselfahrzeugen.

\section{Struktur}

Nach eigenen Angaben hatte Greenpeace 2009 weltweit etwa drei Millionen Fördermitglieder (Greenpeace 2010). Greenpeace Deutschland kam 2009 auf etwa 562.000. In der deutschen Zentrale in Hamburg arbeiten etwa 206 MitarbeiterInnen auf 160 Stellen. International beschäftigt Greenpeace etwa 1.200 feste MitarbeiterInnen. Die umweltpolitischen Themen, mit denen sich Greenpeace aktuell befasst, sind Energie, Klima, Atomausstieg, Wälder, Meere, Gentechnik, Landwirtschaft, Chemie und Globalisierung (Greenpeace 2010). Greenpeace Deutschland ist damit eines der weltweit 28 nationalen und regionalen Büros, die größtenteils autonom agieren sollen. Mit dem Hauptsitz in Amsterdam ist Greenpeace in mehr als 40 Ländern vertreten (GREENPEACE 2008). Greenpeace verfügt über einen Aufsichtsrat und eine Geschäftsführung. Eine wichtige Rolle spielen aber auch die Fördermitglieder, welche die Organisation finanzieren, sowie die ca. 90 Greenpeace-Gruppen (inklusive der Jugendarbeitsgruppen (JAG) und des Team50plus). Es besteht ein Vertrag zwischen Greenpeace International und Greenpeace Deutschland, in dem Gewaltlosigkeit, Überparteilichkeit, die Unabhängigkeit von Staaten und Unternehmen, sowie die Teilnahme an internationalen Kampagnen vereinbart werden (MARKHAM 2008). Laut Greenpeace International gab es 2009 etwa 3 Millionen UnterstützerInnen weltweit mit einem Spendenaufkommen von 195,9 Millionen Euro. Der Großteil

\footnotetext{
${ }^{1}$ Ein bekanntes Beispiel dafür ist die Kampagne gegen die geplante Versenkung der Ölplattform Brent Spar im Nordatlantik im Jahre 1995 (WALlRAFF 2010).
} 
der SpenderInnen und des Spendeneinkommens stammen dabei aus Deutschland (GREEnPEACE 2009).

Die zentralistische und hierarchische Struktur, durch die sich Greenpeace besonders in den 1970ern und 1980ern von den anderen, häufig basisdemokratisch ausgerichteten, Organisationen abhob, ist ein Kritikpunkt. Dies bezog sich sowohl auf die zum Teil mangelnde Einbindung von ehrenamtlichen, sowie auf die starke Hierarchie und die geringe Mitsprachemöglichkeit der Fördermitglieder (Voss 2007). MARKham (2004: 14) fasst zusammen:

„Greenpeace bemüht sich nicht um die Massenmobilisierung von Aktivisten; seine lokalen Gruppen sind klein und werden von der Zentrale stark kontrolliert. Schließlich, obwohl Greenpeace auch Lobbyarbeit betreibt, ist es seine Hauptaufgabe, das Umweltbewusstsein durch spektakuläre Aktionen zu erhöhen."

In diesem Sinne könne Greenpeace nicht als eine klassische Mitgliederorganisation bezeichnet werden und es sei strittig, ob es sich dabei um eine soziale Bewegungsorganisation handele, so Voss (2007). Laut MARKHAM (2008) werden von Greenpeace als Argumente für die sehr zentralisierte Struktur eine höhere Effektivität, kürzere Entscheidungszeiten, die Größe der Organisation und die internationale Ausrichtung genannt. Bezeichnend war die Abspaltung von einigen AktivistInnen, die 1982 zur Gründung von Robin Wood führte. Diese Organisation entwickelte sich aus einer Kritik an den Strukturen von Greenpeace zu einer lockerer organisierten, etwas „radikaleren“ Organisation (MARKHAM 2008).

Greenpeace ist von seiner Struktur nicht unbedingt eine typische Umweltorganisation in Deutschland. Wie für den internationalen Zusammenhang, werden die Hierarchien als häufiger Kritikpunkt genannt. Dies sagt auch etwas über die Form der Zusammenarbeit und Mitbestimmung aus. Auf der anderen Seite gilt Greenpeace als eine sehr unabhängige Organisation und es wird viel Aufwand betrieben dieses Image zu erhalten. Die Aktionen sind sehr genau und zielorientiert geplant und fast immer auf Medienwirksamkeit ausgerichtet. Dabei stellt sich die Frage, wie Greenpeace den öffentlichen Umweltdiskurs beeinflusst und die eigene Sichtweise verbreitet. Die große Bekanntheit trägt sicherlich dazu bei, unterschiedliche Formen von Unterstützung zu erlangen, seien dies SpenderInnen, passive Unterstützung oder aktive Mitglieder. Die Strategien und Kampagnen erscheinen in einigen Punkten etwas selektiv. Es bleibt unklar, nach welchen Kriterien diese ausgewählt werden und wer darüber bestimmt. 


\subsubsection{Deutscher Jungendbund für Naturbeobachtung}

Über den Deutschen Jungendbund für Naturbeobachtung (DJN) gibt es keine wissenschaftlichen Publikationen. Die Informationen für die Beschreibung des Vereins stammen von der Website des DJN, aus der Vereinssatzung und der Geschäftsordnung.

Der DJN wurde 1950 in Böhmsholz bei Lüneburg gegründet und hat seinen Sitz als eingetragener Verein in Göttingen. Das Alter der Mitglieder im Verein ist auf zwölf bis 25 Jahre beschränkt (DJN 2010). In seinem Selbstverständnis legt der DJN besonderen Wert auf Bildung: „Wer die Welt verändern will, muss sie erst verstehen lernen!" (DJN 2010a). Schule und Elternhaus bereiten ihrer Ansicht nach unzureichend auf eine „nachhaltige“ Nutzung der Natur vor. Der DJN bezeichnet sich selbst als „naturverbunden“ und ohne „hierarchische Vereinsstrukturen“. Außerdem wird betont, dass er unabhängig, basisdemokratisch, überparteilich und selbstorganisiert sei (DJN 2010a). In der Satzung werden als Ziele des DJN in Artikel 2 festgehalten (DJN 2010:1):

„Bei seinen Mitgliedern Naturverbundenheit und Kenntnisse über ökologische Zusammenhänge zu vermitteln und zu vertiefen. Aktive Naturund Umweltschutzarbeit leisten. Den Natur- und Umweltschutzgedanken in der gesamten Bevölkerung zu verbreiten und diese zu ökologischen[!] sinnvollem Handeln aufzufordern."

Die Ziele bleiben dabei verhältnismäßig abstrakt. Der Gedanke des „Vermittelns“ scheint einen hohen Stellenwert zu haben und findet sich explizit auf der Website (DJN 2010a) und in den Interviews wieder. Unklar ist dabei, was konkret unter Natur- und Umweltschutz verstanden wird und welche Ziele damit verfolgt werden sollen. Fraglich ist auch, was unter „ökologisch sinnvollem Handeln“ verstanden wird. Ein letzter Punkt ist die Vermittlung von "Naturverbundenheit“. Hier bleibt offen, was unter Natur verstanden wird und welche Form von „Verbundenheit" gemeint ist. Die Aktivitäten, um diese Ziele zu erreichen, werden in Artikel 4 genannt (DJN 2010:2):

- „Naturkundliche und daraus folgende umweltpolitische Arbeit, Durchführung gemeinsamer Veranstaltungen wie Lager, Exkursionen, Gruppenabende usw.

- Bildung spezieller Arbeitsgemeinschaften auf naturkundlichem und umweltpolitischem Gebiet.

- Herausgabe von Veröffentlichungen auf naturkundlichem Gebiet.

- Erteilung von Auskünften auf dem Gebiet der Naturkunde und des Umweltschutzes. 
- Praktische Mithilfe im Natur- und Umweltschutz.

- Gründung neuer Gruppen und Aufnahme bereits bestehender Gruppen mit ähnlichen Zielen.“

Es ist ein Schwerpunkt im Bereich der Naturkunde erkennbar, der die Verbreitung von Informationen und Bestimmungsschlüsseln einschließt. Außerdem wird die umweltpolitische Arbeit hervorgehoben, die aus der naturkundlichen Arbeit folgen soll. Auf Bundesebene gibt es, vereinsrechtsgemäß, einen Vorstand, der aus Bundesvorsitz, NaturkundesekretärIn, LagersekretärIn, AuslandssekretärIn, KassenwartIn, WerbesekretärIn und PressesprecherIn besteht. Bei Abstimmungen gelten mehrheitliche Entscheidungen (DJN 2010b). Der DJN vertreibt Bestimmungsliteratur (z.B. für Farne, Libellen, Käfer) und das naturkundliche Magazin „Naturkundliche Beiträge“, in dem z.B. Ergebnisse von Projekten veröffentlicht werden (DJN 2010c). Es gibt regelmäßig selbstorganisierte Aktivitäten, wie z.B. Radtouren, Klettertrainings oder Seminare wie „Eigenmacht und Selbstverantwortung in Gruppenstrukturen“ (DJN 2010d).

Insgesamt lassen sich drei Schwerpunkte festhalten, die für den DJN charakteristisch sind: Erstens arbeitet der DJN sehr naturkundlich. Es gibt Bestimmungsliteratur, die stetig erweitert wird und einen wissenschaftlichen Anspruch hat. Exkursionen zu naturkundlichen Themen und praktischem Naturschutz ergänzen dieses Bild. Zweitens wird viel Wert auf die Vermittlung von Wissen gelegt, dies wird im Selbstverständnis des DJN immer wieder betont. Wie die Zitate oben gezeigt haben, gehe es darum die Welt zu verstehen und bei den Mitgliedern „ökologische Zusammenhänge zu vermitteln und zu vertiefen“. Der dritte Punkt betrifft die politische Arbeit: Der DJN hat einen politischen Anspruch. Es gehe darum, Hierarchien abzubauen, selbstorganisiert zu arbeiten und basisdemokratisch zu entscheiden. Außerdem sollen, wie eingangs diskutiert, Umwelt- und Naturschutzgedanken in der gesamten Bevölkerung verbreitet werden. Dies ist eine politische Aussage, auch wenn offen bleibt, welche Ziele im Umwelt- und Naturschutz verfolgt werden sollen.

\subsection{Auswertung und Interpretation der Interviews}

In diesem Kapitel erfolgt die Auswertung der Interviews. Das Kapitels ist entsprechend der Auswertungskategorien, die sich aus der qualitativen Inhaltsanalyse ergeben haben aufgebaut (siehe Aufbereitungsverfahren 4.2.5)). Bei den Quellenangaben handelt es sich jeweils um den Interviewcode (siehe Anhang A.2) und die entsprechende Zeilennummer(n) Zwischen den Themen gibt es einige

\footnotetext{
${ }^{2}$ Bei indirekten Zitaten wird damit das Ende der entsprechenden Textpassagen angeben. Dies ließ sich aus technischen Gründen leider nicht anderweitig umsetzen. Die vollständigen Interview-
} 
Überschneidungen, so dass zu Anfang eines Abschnitts darauf hingewiesen wird, welche Aspekte schwerpunktmäßig berücksichtigt werden. Wie bei GLÄsER \& LAUdEL (2009: 272) beschrieben, wurde hier ein Kompromiss zwischen einer Aggregation von Daten in verarbeiteter Form und der Auswahl von Daten als direkte Zitate gewählt. In diesem Kapitel wurde darauf Wert gelegt, die Aussagen der InterviewpartnerInnen nicht in einen zu ,verwissenschaftlichten“ Kontext zu stellen, um eine größere Nähe zu ihnen herzustellen. Aus diesem Grund ist die Wortwahl stellenweise etwas umgangssprachlicher.

\subsubsection{Die Organisationen}

Im Laufe der Interviews wurden einerseits einige Fakten zu den Organisationen erfragt, d.h. Fragen nach Mitgliederzahl und Aktivitäten, andererseits auch um eine Einschätzung der Organisationen und Mitglieder. Dieses Kapitel ergänzt somit die Beschreibungen der Organisationen durch die Literatur (siehe Beschreibung der untersuchten Organisationen (5.1)).

\section{Deutscher Jungendbund für Naturbeobachtung}

Insgesamt habe der Verband etwa 100 Mitglieder, die an Veranstaltungen des DJN teilnehmen (z.B. dem Besuch von Seminaren), davon seien etwa 30-50 aktive Mitglieder, die sich freiwillig engagierten (Int1:131). Nach Angaben eines Interviewpartners lag die Zahl früher teilweise bei 500 Mitglieder (Int1:134). Es gebe Ortsgruppen in mehreren deutschen Städten, von denen Göttingen die Geschäftsstelle des Vereins darstellt und als ",aktivste Ortsgruppe“ (Int1:139), mit ungefähr 10 regelmäßig aktiven Mitgliedern, bezeichnet wurde (Int1:141). Die Gruppen in Süddeutschland seien zunehmend geschrumpft oder weggefallen (Int6:88). Allerdings habe der DJN immer wieder Engpässe in den Mitgliederzahlen gehabt und hatte mit der Aufrechterhaltung der Strukturen zu kämpfen (Int6:74). Auf Bundesebene gebe es einen Vereinsvorstand, der die Arbeit mit den anderen Gruppen regele, für das Vereinsrecht zuständig sei und Seminare auf Bundesebene organisiere (Int1:153). Es gebe eine Jahresplanungs-AG und Kongresse, an denen alle Mitglieder teilnehmen (Int1:92,110). Die Arbeit mit anderen Gruppen werde auch vom Bundesvorstand organisiert (Int1:164). Es werde Wert darauf gelegt, die Bundesorganisation mit ihren Abstimmungen an jüngere Mitglieder zu vermitteln (Int6:236).

Nach eigenen Angaben führt der DJN Seminare, Exkursionen und Freizeiten mit naturkundlichen oder umweltpolitischen Schwerpunkten durch (Int1:20). Es werden Bestimmungsschlüssel für Pflanzen und Tiere herausgegeben (Int1:21)

transkripte finden sich auf einer CD im Anhang dieser Arbeit. 
und mit unterschiedlichen PartnerInnen Projekte durchgeführt (auch auf internationaler Ebene) (Int1:81). Ursprünglich habe sich der DJN aus der AntiAtomkraftbewegung entwickelt, so eine Interviewpartnerin (Int6:45). Generell sei der Verband „naturkundlich geprägt“ (Int1:27) und betreibe praktischen Naturschutz. Allerdings sei der Naturschutz in der Vereinsgeschichte teilweise zugunsten politischer Themen in den Hintergrund getreten oder die Aufrechterhaltung der Strukturen und die Organisation nahmen viel Zeit in Anspruch (Int6:459).

Die Mitglieder bezeichnen sich im DJN als gleichberechtigt und die Vorstandsmitglieder seien etwas aktiver (Int1:156). Die verschiedenen Ortsgruppen hätten unterschiedliche Schwerpunkte und seien mal politischer und mal naturkundlicher geprägt (Int6:54). Die begrenzte Altersspanne von zwölf bis 25 Jahren werde von den Mitgliedern als positiv wahrgenommen und der Verein habe dadurch eine „jugendliche Struktur“ (Int6:96). Neue Mitglieder werden viel durch „Mund-zu-Mund-Propaganda“ (Int6:149), durch Aktionen (Int6:150) und durch Flyer, Zeitungen oder das Internet (Int6:141) gewonnen.

\section{Greenpeace}

Nach eigenen Angaben verfüge Göttingen über eine relativ große GreenpeaceGruppe. Es gebe eine Erwachsenengruppe, eine Jugendarbeitsgruppe und ein Team50plus, das allerdings aktuell nur aus drei bis fünf Mitgliedern bestehe (Int4:581). Ferner gebe es einen Presseansprechpartner (Int3:75), einzelne ThemenansprechpartnerInnen, sowie einen Gruppenkoordinator, der einen Überblick über Finanzen, Mitglieder und Themen der Gruppe habe (Int4:98). Die Gruppe wurde als sehr „studierendenlastig“ beschrieben und die meisten Mitglieder brächten sich etwa ein bis zwei Jahre aktiv ein (Int4:70,103).

Auf übergeordneter Ebene werden die Göttinger Gruppen von der Zentrale von Greenpeace Deutschland e.V. in Hamburg koordiniert (Int3:95). Die Zentrale versorge die Gruppen mit Materialien zu Themen und mit Geldern für Aktionen (Int3:64; Int5:109). Die Beziehung zu Hamburg wurde unterschiedlich eingeschätzt. Die Organisation wurde als relativ „strikt vorgegeben“ (Int3:74) bezeichnet oder es bestehe „in Göttingen ein bisschen das Problem, dass wir uns viel von Hamburg leiten lassen, .. weniger eigenständige Projekte haben.“ (Int4:288-289). Allerdings werden auch regionale Aktivitäten von der Zentrale gefordert (Int3:100; Int4:268,277) und es wurde geäußert, dass durch mehr regionale Projekte eine höhere Unabhängigkeit von der Zentrale erreicht werden könne (Int3:358), so zum Beispiel durch ein langfristiges und größeres Team50plus mit mehr Kontakten in die Region (Int4:573).

Weiter wurde Greenpeace beschrieben als eine „Organisation, die allumfassend ist, die alle Generationen nimmt und eigentlich alle Umweltthemen“ (Int5:535-536) behandle. Insbesondere die Vielfalt an Themen und die Altersstruktur mit den unter- 
schiedlichen Gruppen wurden in den Interviews als positiv hervorgehoben (Int3:44; Int5:520,629), so berichtet eine Interviewte:

„[...] wenn man Greenpeace sagt, fallen mir zuerst die Leute ein und dann die Themen .. dann wahrscheinlich auch die Struktur, also dass wirklich von Kindern bis Erwachsenen bis alten Leuten alles gibt." (Int5: 627-629)

In den Greenpeace-Interviews zeigt sich, dass die Unabhängigkeit von Greenpeace von allen Interviewten geschätzt wird (Int3:41,334; Int4:232; Int5:304). Dies wird durch die Selbstdarstellung von Greenpeace auf den Webseiten und Flyern (siehe 5.1.1) bestärkt und wird in den Interviews sowohl als Eigenschaft von Greenpeace als auch als Motiv für die Mitarbeit hervorgehoben,

„[...] dass es unabhängig ist, also dass es eine Organisation ist, die sich nur aus Spendengeldern finanziert, dabei wie ich finde, kann man alles machen, was man möchte. Man braucht nicht darauf achten, wenn man jetzt irgendwie auf die Füße tritt [...]." (Int3:40-43)

Greenpeace sei groß und einflussreich und nehme eine Vorreiterrolle bei den Umweltorganisationen ein (Int5:517,520). Ein wichtiger Punkt, der auch in der Literatur diskutiert wird, ist die Glaubwürdigkeit von Greenpeace. Eine Interviewte schilderte:

„Greenpeace wirkt halt von seiner Glaubwürdigkeit, aber im Moment denke ich, sieht es halt ganz gut aus mit der Glaubwürdigkeit und ich denke schon, dass viele Menschen auch darauf achten, so ein bisschen, was Greenpeace sagt, und von daher denke ich, dass wir da gute Chancen haben in Zukunft da auch einiges zu bewegen." (Int3:359-363)

Große Bekanntheit hat Greenpeace durch seine Aktionen und Kampagnen erlangt. Die meisten Aktionen seien relativ einfach umzusetzen und praktisch orientiert (Int3:213,334). Es gebe kleine Aktionen, die von den regionalen Gruppen organisiert werden und Gruppenaktionstage, die von der Zentrale organisiert werden und an denen Gruppen aus vielen verschiedenen Städten teilnähmen (Int3:81; Int5:188). Diese verliefen, nach Angaben einer Interviewten, meist erfolgreich (Int5:163,191). Die Aktionen werden geheim geplant, so dass die Presse vorher nicht davon erfahre (Int5:166). Insbesondere in den Erwachsenengruppen werden Infostände häufig angewandt (Int5:41), mit dem Ziel, dass man „Bürger direkt ansprechen kann“ (Int3:211-212). Die Beteiligten seien von Greenpeace während der Aktionen versichert (Int4:100). Betont wurde, dass Greenpeace nicht zu Demonstrationen aufrufe (Int4:222). Hinsichtlich der Strategien für die Aktionen merkt ein Interviewter an: 
„Also man möchte natürlich immer erst Gespräche suchen. Man wird erst dann konfrontativ, wenn man merkt, Gespräche haben keinen Sinn. Erst dann geht man ja wirklich in Konfrontation und das sollte man natürlich auch immer beibehalten." (Int4:633-635)

Diese Konfrontationen können über Druck aus der Bevölkerung (z.B. Aufruf zum Konsumverzicht, Informationspolitik durch Aufmerksamkeit erregende Aktionen) oder über die Politik (z.B. durch Gutachten, Recherchen) ausgeübt werden (Int4:641; Int5:518). Ein weiteres Mittel sei z.B. das Befestigen von großen Bannern an Industrieanlagen. Dabei ständen konkrete Forderungen und Ziele im Vordergrund. Die Gewaltfreiheit von Protesten werde dabei als "ganz wichtig“ (Int4:628) angesehen. Dabei werde vorrangig auf Medienwirksamkeit abgezielt: „Also, es ist schon alles sehr stark auf Medien ausgerichtet, gerade auf bildliche Medien, Tageszeitungen." (Int5:604-605). Dies wird zum Teil allerdings als problematisch wahrgenommen, weil Aktionen dadurch stark auf die Wirkung in Fotos oder Videos abgestimmt werden (Int5:613). In den Interviews wurde vorgeschlagen, dass es manchmal sinnvoller sei, noch konfrontativer zu arbeiten (Int4:626) oder alternative Strategien auszuprobieren (Int4:626). Außerdem seien langfristige Aktionen hinsichtlich der Organisation und der Motivation schwer umzusetzen (Int5:175). In zwei Interviews wurde darauf hingewiesen, dass es neben der Arbeit in den Gruppen noch die Möglichkeit gebe, sich als AktivistIn zu engagieren, dies erfordere allerdings Fähigkeiten, wie Klettertechniken, Bootfahren, etc. (Int4:o.Aufn.; Int5:244). Diese seien Bestandteil von Weiterbildungsangeboten, die Greenpeace zur Verfügung stelle (Int3:303). Darunter fallen auch Workshops zur regionalen Kampagnenarbeit, zur Koordination von Gruppen oder Medieneinsatz (Int4:86,271). Des Weiteren gebe es regelmäßige Fachbereichstreffen, in denen Themenschwerpunkte und langfristige Strategien diskutiert werden (Int4:90).

Es zeigt sich, dass Greenpeace und der DJN sehr unterschiedliche Strukturen aufweisen. Bei Greenpeace handelt es sich um eine große, international agierende Organisation mit relativ festen Hierarchien, Themenschwerpunkten und langfristigen Strategien. Es gibt nach Alter gestaffelte Gruppen und Budgets für Aktivitäten. Viele Themen werden von der Zentrale vorgegeben, die dafür geeignetes Material und Weiterbildungsangebote zur Verfügung stellt. Das kann auf Kosten der Flexibilität der Gruppen gehen, z.B. was die Pressewirksamkeit oder Kooperationen mit anderen Gruppen betrifft. Der DJN ist auf der anderen Seite als ein Verein organisiert, in dem Mitbestimmung auch auf Bundesebene direkt stattfinden kann und Mitglieder sich (auch aufgrund der geringen Zahl an Mitgliedern) relativ schnell beteiligen können. Weiterbildungen oder Themenwahl erfolgen dabei eher eigeninitiativ. 


\subsubsection{Motivation}

In diesem Abschnitt wird die Auswertungskategorie Motivation behandelt. Zum einen geht es darum, wie die Mitarbeit in den Vereinen zustande gekommen ist und zum anderen, was die Wahl einer konkreten Organisation beeinflusst hat. Ein weiterer Punkt ist die Motivation für die langfristige Teilnahme.

\section{Der Weg zur Organisation}

Zunächst wurden die Interviewten gebeten zu schildern, wie der Einstieg in die Organisationen für sie ausgesehen hat. Dieser sagt zum einen etwas über die Motive aus, warum sich Menschen engagieren und zum anderen darüber, wie der Einstieg in die Organisationen erfolgt, z.B. wie jemand davon erfahren hat oder wie das erste Treffen ablief. Eine Interviewte bei Greenpeace schilderte:

„[... a ab dem dritten Semester, also, habe ich mir in Selbstinitiative auch gesucht, ob ich hier halt, ob ich hier eine Umweltorganisation finde in Göttingen, weil ich mich halt gerne aktiv irgendwo einbringen wollte und hab dann daraufhin Greenpeace gefunden und das war ein bisschen auch Glücksentscheidung zunächst, weil halt die Plenumszeit genau in meinem Stundenplan passt. Dann bin ich da halt einmal hin und das hat mir auf Anhieb super gut gefallen, weil ja, total nette Leute und ja dann bin ich auch dabei geblieben." (Int3:19-25)

Die verfügbare Zeit für das Engagement ist ein wichtiger Faktor und ob sich die Personen bei den ersten Treffen wohl gefühlt haben. Eine andere Interviewte beschrieb:

„Also in dem Moment in dem ich gesagt habe, ich will zu Greenpeace gehen und nicht zur Grünen Jugend, lag einfach daran, dass ich da Leute kannte und ich wusste wie lange die da brauchen, bis sie ihre Aktion auf die Beine gestellt hatten." (Int5:297-299)

Freunde und Bekannte, die selbst aktiv sind, waren bei dieser und anderen interviewten Personen ausschlaggebend für einen Einstieg. Außerdem wird hier auch die Arbeitsweise betont, die der Aussage nach bei Greenpeace effektiv sei. Die große Bekanntheit von Greenpeace trägt auch dazu bei, dass Menschen sich gezielt für diese Organisation entscheiden:

„[...] ich müsste im Umweltschutz was machen und ja eigentlich mit der Brand Spar, als die gesunken ist, [...] wo ich gedacht hab, das was Greenpeace da macht finde ich gut, finde ich richtig, das würde ich irgendwie gerne unterstützen. Damals war das nicht so machbar, das war auch immer relativ weit weg. Und Göttingen hatte eben eine GreenpeaceGruppe und da habe ich gesagt, ok, da muss ich mal hingehen und mir 
das angucken. So bin ich dann eigentlich zu Greenpeace gekommen." (Int4:23-29)

Hier zeigt sich eine der Strategien von Greenpeace, durch öffentlichkeitswirksame Aktionen Unterstützung zu erlangen. Der Interviewpartner schilderte dies als ausschlaggebendes Moment für seinen Einstieg.

Betrachtet man den DJN, so erfolgt der Einstieg stärker über Bekannte oder Verwandte:

„[...] eine Freundin von meinem Mitbewohner hat davon erzählt und dann bin ich mitgegangen und irgendwie hat das mich mehr mitgerissen, als die Grüne Hochschulgruppe. Da war ich zu dem Zeitpunkt schon relativ lange, so zwei drei Jahre. Ich fand das irgendwie spannender, ein bisschen greifbarer." (Int1:61-64)

\begin{abstract}
„Also, ich bin da eigentlich ein bisschen rein geboren in den Verein, also meine Eltern waren da schon drin und mein großer Bruder auch und der wollte mich immer mitnehmen und vor allem seine Kumpels und ich hatte am Anfang immer ein bisschen Angst." (Int6:10-12)
\end{abstract}

Neben diesen Punkten führten die Interviewten noch an, dass sie über den Einstieg viele „Gleichgesinnte“ getroffen hätten (Int3:174; Int6:390,520) und sie die Arbeit in den Gruppen motiviert habe (Int1:62,102). Der Einstieg kann als Schlüsselaspekt für die Partizipation bezeichnet werden, da von ihm abhängt, ob eine längerfristige Teilnahme stattfindet. Festhalten lässt sich, dass der Zugang zum einen stark eigeninitiativ erfolgte, das heißt, es wurde konkret nach Organisationen gesucht und zum anderen über Freunde und Bekannte, die einen Einstieg erleichtern. Es ist davon auszugehen, dass mehr Personen gezielt zu Greenpeace gehen, weil diese Organisation im Gegensatz zum DJN sehr bekannt ist.

\title{
Motivation in Bezug auf Natur und Umwelt
}

Da es sich um Umweltorganisationen handelt, ist es interessant, welche Rolle Natur bzw. Umwelt für die Motivation spielt. In den DJN-Interviews wurden besonders das Interesse an Themen, wie Artenvielfalt, Naturschutz oder die Funktion von Ökosystemen genannt (Int1:88,99,179; Int2:94). Ferner wurde die Unterstützung des Naturschutzes (Int1:300; Int6:454) und der Aufenthalt in und das Erfahren von Natur hervorgehoben (Int6:456). Diese Motive finden sich teilweise in den Greenpeace-Interviews, allerdings wurden die Aspekte bei Greenpeace nicht so stark betont wie beim DJN. Es überwog eher die Einstellung, dass Umweltschutz wichtig und die Welt für kommende Generationen zu erhalten sei (Int3:318; Int4:24,335). 
Hier wäre es interessant benennen zu können, was genau unter den Begriffen „Umwelt" und „Natur" bzw. „Umweltschutz" und „Naturschutz" verstanden wird. Im Vergleich zeigt sich, dass in den Interviews mit Greenpeace-Mitgliedern häufiger die Begriffe „Umwelt“ und „Umweltschutz“ gebraucht werden. Bei DJN-Mitgliedern waren dies eher die Begriffe „Natur“ und „Naturschutz“. Auch das „Kennenlernen“ und „Erleben“ von Natur steht bei den DJN-Interviews eher im Vordergrund, wohingegen bei Greenpeace die Schwerpunkte auf (technischeren) Themen, wie Gentechnik, Meeresschutz oder Energie liegen.

\section{Motivation in Bezug auf die Arbeit}

In den Interviews wurden viele Gründe angeführt, warum sich Menschen in Umweltorganisationen beteiligen, doch es lassen sich einige Punkte festhalten, die stärker betont wurden. Zunächst sind das Lernen und das Vermitteln von Wissen ein Aspekt, der in einem Interview des DJNs sehr hervorgehoben wurde: „Ja, mein Lebensmotto ist ,ich lebe um zu lernen' und ja, das treibt mich da auch so ein bisschen an“ (Int2:116-117). Weiter hieß es:

„Das ist, also, ja, mir ist es eben ganz wichtig, das Wissen weiterzuleiten, wenn ich jetzt von wem was neu kennengelernt oder gehört habe, dann will ich das so schnell wie möglich andern weitersagen, damit die es wiederum anderen weitersagen können [...]." (Int2:104-106)

Der DJN bietet einen Rahmen, in dem Wissen erworben werden kann und in dem es Möglichkeiten gibt, Wissen an andere weiterzugeben. Dieser Austausch von Wissen wird vom Interviewpartner als erstrebenswert empfunden. Ein weiterer Punkt ist die Arbeitsweise im DJN, welche motivierend wirke. Ein Interviewpartner berichtete:

„[...] aber das schon jetzt so im Nachhinein, weil ich so geprägt worden bin, ich aber schon auch wieder den DJN auswählen würde, gerade wegen dieser Struktur, dass jeder sich einbringen kann, jeder einfach seine Ideen umsetzen kann, nicht lange warten muss, auf irgendwelche Genehmigungen oder so. Und auch wenn mit 25 Schluss ist und ich finde das ist ein ganz spannender Prozess, diese Altersbegrenzung und versuche diese auch zu verteidigen gegen andere Strömungen." (Int1:346-351)

Hier wird hervorgehoben, dass jeder sich „einbringen kann“ und „Ideen umsetzen kann“. Diese Punkte werden in den DJN-Interviews immer wieder positiv hervorgehoben (z.B. Int1:30,160; Int2:66,77) und sie scheinen im DJN eine große Rolle zu spielen. Dies trifft auch auf die besondere Altersmischung zu, die eine Besonderheit des DJN darstellt. Mitglieder sind zwischen zwölf und 25 Jahren alt, aber innerhalb 
dieser Altersspanne variiert das Alter sehr, so dass Jüngere mit Älteren zusammenarbeiten ${ }^{3}$. Als dritter Punkt wurde die persönliche Beziehung hervorgehoben, welche zwischen den Mitgliedern des DJN bestehe: „es ist einfach so ein relativ entspannter und gesellschaftlicher Umgang mit anderen Leuten, aber das ist irgendwie auch so ein Typ Mensch“ (Int6:389-390). In einem anderen Interview hieß es: „Die Leute, oder die Personen. Da freundet man sich halt mit der Zeit an und dann kennt man sich schon und lädt sich gegenseitig ein und das ist immer ganz schön." (Int2:194195) Auch im ersten Interview wird gesagt, dass „inzwischen ein Großteil meines Freundeskreises im DJN verwurzelt ist" (Int1:55). Das Treffen mit Gleichgesinnten, Freundinnen und Freunden sei eine große Motivation für das Engagement im DJN. Dies geht sogar so weit, dass in Zeiten mit wenigen Mitgliedern der Verband durch Freundschaften erhalten werden konnte, so eine der Interviewten (Int6:469-470). Als weitere Punkte, die zur Mitarbeit motivierten, wurden aufgeführt: Die Möglichkeit „eigene“ Sachen zu machen und dadurch unabhängig zu sein (Int1:210,213), der gute Umgang miteinander (Int6:291,390) und dass einem der Verband „ans Herz“ (Int6:293-294) wachse.

Die Mitglieder von Greenpeace betonen abweichende Motive für ihr Engagement. Hier ist der Aspekt der Veränderung häufig präsent: „Meine Motivation für mich ist eigentlich, dass ich was verändern möchte und vor allen Dingen auch aktiv was verändern möchte.“ (Int4:317-318) Eine andere Interviewte sagte,

„[...] dass man halt auch wirklich das Gefühl hat, man trägt was dazu bei, was zu verbessern. Das man was bewegen kann. Das ist mir halt persönlich halt auch sehr wichtig, dass ich nicht nur zugucke, sondern dass ich halt aktiv was verändere." (Int3:227-230)

Der Wille, aktiv etwas zu verändern, ist eine starkes Motiv bei den Interviewten von Greenpeace, dies betrifft auch die Kommunikation von Inhalten an Außenstehende: „Das ist eigentlich das, was ich möchte, Leute zum nachdenken anregen und dann eben auch wieder zum handeln in irgendeiner Form, was auch immer das ist" (Int4:428-430). Als weitere Motive tauchten noch die „spannenden“ und internationalen bzw. überregionalen Themen auf (Int4:49-52; Int5:261,316) und die zielorientierte Arbeit an konkreten Aktionen (Int3:34; Int5:286,289).

Der Einstieg in den DJN erfolgt häufig über Freunde und Bekannte. Für die langfristige Mitarbeit wurden allerdings noch andere Aspekte genannt: In den DJNInterviews fällt auf, dass das Lernen und Vermitteln eine große Rolle spielt und für die Interviewten ein Antrieb für die Teilnahme ist. Außerdem ist die persönliche Beziehung zueinander ein wichtiges Motiv. Innerhalb des Vereins haben sich Freundschaften gebildet und das Zusammensein mit „Gleichgesinnten“ hat einen hohen

\footnotetext{
${ }^{3}$ Auf die Erfahrungen mit der Altersstruktur wird in dem Abschnitt Altersstruktur 5.2.6 näher eingegangen.
} 
Stellenwert. Ein weiterer Punkt ist, dass alle Mitglieder die Möglichkeit haben sollen, sich einzubringen. In den Greenpeace-Interviews war auffällig, dass häufig das Wort „verändern“ fiel. Gesellschaftliche Veränderungen herbeizuführen scheint einen hohen Stellenwert bei den Mitgliedern zu haben. Insgesamt überschneiden sich die Motive für die Teilnahme, aber es lässt sich dennoch erkennen, dass die Schwerpunkte bei Greenpeace und im DJN verschieden sind. 


\subsubsection{Partizipation}

Weitere Fragen in den Interviews betrafen die Partizipation. Die Interviewten wurden gebeten den Begriff zu definieren oder zu umschreiben. Anhand ihrer Definitionen sollte geklärt werden, was unter Partizipation verstanden wird, um Rückschlüsse auf das Verhalten innerhalb der Gruppe treffen zu können. Durch weiterführende Fragen war es dann möglich auf die Themen Erlernbarkeit von Partizipation, förderliche und hinderliche Faktoren für die Teilnahme und Vorschläge für eine stärkere Einbeziehung von Mitgliedern zu erfahren. Bei den Fragen nach Partizipation wurde Wert darauf gelegt, diese nicht an den Anfang zu stellen, damit an dem vorher gesagtem angeknüpft werden kann. Im Gegensatz zum Abschnitt über Motivation, wird in diesem Kapitel stärker auf die strukturellen Aspekte und das Einbinden von Mitgliedern eingegangen. Motivation und Partizipation lassen sich jedoch nicht vollständig trennen.

\section{Sichtweisen von Partizipation}

Da Partizipation auf unterschiedliche Weise definiert wird (siehe 3.1), ist es zunächst interessant, was die Interviewten, als aktiv Beteiligte, darunter verstehen. Daraus ergeben sich dann Teilfragen, auf die genauer eingegangen werden kann. Die Interviewten von Greenpeace beschrieben:

„[... ] ich würde sagen, man kann es erfahren und dadurch ist wahrscheinlich mehr verinnerlicht auch und dann weitergeben. Aber ich würde jetzt nicht sagen, dass man das irgendwie aktiv lernen kann, ich würde eher sagen man erfährt es, zum Beispiel in einer Gruppe in der man dann partizipiert oder teilnimmt und etwas erfährt oder Erfahrungen macht und kann halt daraus dann auch was lernen. Es ist schwierig. Ich würde jetzt nicht sagen, man kann es direkt lernen, aktiv, aber durch Erfahrungen kann man etwas dazulernen." (Int3: 245-251)

„Man ist immer selber Teil davon, man muss immer an Aktivitäten teilnehmen. Man kann nicht sagen ,macht mal'. Das funktioniert nicht." (Int4: 518-520)

„Ja .. dass man an etwas teilnimmt, dass man sich für etwas engagiert. Partizipation ist, glaub ich, auch immer in der Gruppe soweit ich weiß, also quasi, dass man sich als Teil einer Gruppe für etwas einsetzt, an etwas teilnimmt." (Int5: 380-382)

Partizipation stellt sich als etwas dar, dass nicht direkt vermittelt, sondern indirekt durch das Teilnehmen in einer Gruppe erfahren werden kann. Somit handelt es sich 
eher um etwas prozesshaftes, als um etwas, dass sich klar benennen oder beschreiben lässt. Das „teilnehmen“ taucht in allen drei Zitaten auft. In dem zweiten Zitat zeigt sich, dass das Übernehmen von Verantwortung eine Rolle spielt. Partizipation sei nur möglich, wenn eine aktive Haltung eingenommen werde. Das letzte Zitat betont einen neuen Aspekt: Es gehe darum, dass man sich als „Teil einer Gruppe für etwas einsetzt". Dafür ist ein gemeinsames Ziel notwendig, welches das Individuum mit der Gruppe teilt.

In den Interviews mit DJN-Mitgliedern wurden teilweise andere Punkte hervorgehoben:

„[...] es geht ja eigentlich darum, wie sehr Mitglieder auch mitgestalten dürfen und was sagen dürfen [...]." (Int6: 404-405)

„Ideen einfach umzusetzen und die halt anzugehen und nicht darauf zu warten, dass die von anderen kommen, sondern sondern selbst das in die Hand nehmen und sich dann selbst darum kümmern und einen Blick dafür zu entwickeln, was alles bedacht werden muss, wenn ich ein Seminar organisiere, wo ich halt Geld beantrage bei so einer Behörde und dann auch damit umzugehen, wenn da Nachfragen kommen oder oder ... noch was, genau, Probleme auftauchen und sich einfach für die eigene Sache einzusetzen, das unterscheidet wahrscheinlich auch den DJN von jetzt anderen Jugendumweltvereinen, die jetzt einen Erwachsenendachverband haben [...]." (Int1: 208-215)

In den Zitaten des DJN werden noch einige Aspekte betont, die in den GreenpeaceInterviews nicht genannt wurden. Die Betonung des „Dürfens“ im ersten Zitat zeigt, dass dies nicht selbstverständlich ist (sei dies im Alltagsleben oder in anderen Vereinen, dies geht aus dem Kontext des Zitats nicht hervor). Der Begriff des „mitgestaltens" hat eine zielgerichtete Konnotation, er enthält eine gewisse Form von Plan oder Vorstellung, auf die hingearbeitet werden kann. In dem zweiten Zitat werden Eigeninitiative, die Übernahme von Verantwortung und selbstbestimmtes Arbeiten hervorgehoben, dies zeigt sich durch die Verwendung von Ausdrücken wie „in die Hand nehmen“, „für die eigene Sache einzusetzen“ oder sich „kümmern“. Gleichzeitig grenzt der Interviewte den DJN zu anderen Strukturen, wie Vereine mit einem Erwachsenendachverband, ab. Außerdem wird im zweiten Zitat noch der Aspekt des Lernens betont. Es gehe darum, einen „Blick zu entwickeln“, also zu Lernen, selbstständig und eigenverantwortlich Projekte zu planen und Aktivitäten durchzuführen. In Verbindung mit Partizipation wurden in den Interviews noch weitere Aspekte angesprochen: Die „gleichen Grundgedanken zu haben“ (Int3:240), an Entscheidungsprozessen teilzunehmen (Int1:249), „Anteilhabe“ zu entwickeln (Int6:203), sich

\footnotetext{
${ }^{4}$ Laut Duden (2000) bezeichnet teilnehmen: dabei sein, mitmachen, Anteil nehmen, teilhaben, bei etwas mitfühlen.
} 
für Themen einzusetzen (Int5:387) oder sich zu engagieren (Int4:560; Int5:380). Einer der Interviewten betonte, dass es Unterschiede zwischen der Arbeitsweise in Betrieben und der freiwilligen Arbeit in Nichtregierungsorganisationen gebe, da sich die Motivation unterscheide (Int4:511), alle sich einbringen möchten (Int4:529) und „bestimmen" auf Ablehnung stoße (Int4:510,520).

Wie deutlich zu erkennen ist, liegen in den Interviews Unterschiede im Verständnis von Partizipation vor: Während das Übernehmen von Verantwortung in beiden Organisationen ein wichtiges Motiv ist, so wird bei Greenpeace eher das aktive Einbringen und Verändern betont, wohingegen beim DJN stärker Gewicht auf individuelle Selbstbestimmung gelegt wird (insbesondere in Abgrenzung zu anderen Verbänden und dem Alltagsleben).

\section{Erlernen von Partizipation}

Einig sind sich alle Interviewten darüber, dass Partizipation bis zu einem gewissen Grad erlernbar sei (Int1:255; Int2:159; Int3:255; Int4:479; Int5:397; Int6:417). Allerdings verlaufe dieser Prozess individuell verschieden (Int2:159; Int6:431), beispielsweise beschreibt einer der Interviewten: „Es gibt Leute, die lassen sich sehr leicht motivieren und arbeiten auch sehr schnell sehr eigenständig, man muss da eigentlich nur so einen kleinen Initialkick geben“ (Int4:127-129). Erfahrungen spielten dabei eine große Rolle (Int1:264; Int3:249,251). Eine der Interviewten schilderte das Lernen von Partizipation als eine, sich selbst steigernde, Kraft:

„[...] wenn man sich irgendwann mal entschlossen hat sich für irgendein Thema einzusetzen, dann wird das dazu führen, dass man sich immer mehr für Themen einsetzt und auch für andere Themen einsetzt und dann an immer mehr Organisationen und immer mehr Projekten teilnimmt." (Int5:401-403)

\section{Schwierigkeiten in Zusammenhang mit Partizipation}

In den Interviews wurde darum gebeten, darzustellen, welche Schwierigkeiten und Probleme in Zusammenhang mit Partizipation gesehen werden und konkrete Situationen dafür zu schildern. Dadurch wird auf der einen Seite das Verständnis von Partizipation vertieft, auf der anderen Seite ermöglicht es Wege zu finden, um eine bessere Beteiligung (sowohl qualitativ, als auch quantitativ) zu erreichen.

Aufgrund seiner Altersstruktur mit einer begrenzten Altersspanne, ist der DJN darauf angewiesen ,in relativ kurzen Abständen immer wieder neue Leute gewinnen, motivieren und einbinden" (Int1:231-233) zu müssen. Organisationen mit einer größeren Bekanntheit dürfte das Finden von neuen Mitgliedern leichter fallen. Um eine langfristige Partizipation zu ermöglichen, sollten die erwähnten Aspekte der 
Motivation (siehe 5.2.2 nicht unterschätzt werden. Im DJN wird betont, dass Jüngere manchmal Schwierigkeiten mit dem Altersunterschied hätten (Int6:177). Außerdem seien Engagierte wichtig, um neue Mitglieder zu finden, da aktiv gesucht werden müsse (Int6:88). Dies sei zum Teil, insbesondere beim DJN in Süddeutschland, versäumt worden (Int6:170). Problematisch sei es, dass neue Mitglieder manchmal Vorgaben erwarteten und die große Entscheidungsfreiheit als schwierig empfänden (Int1:259). Dies wurde in ähnlicher Weise in einem Zitat aus der Greenpeace-Gruppe geschildert:

„[... ] da ist dann ein ganz großes Maß an Eigeninitiative, das man dann braucht, um sich da einzuarbeiten, bis man dann wirklich auch den Mut hat, diese Sachen, die man grade gelernt hat an Leute weiterzugeben." (Int5:459-461)

Zu den Schwierigkeiten, die in beiden Organisationen gesehen werden, zählt auch der Mangel an Zeit bzw. der Zeiteinteilung: Gerade in Semester- und Schulferien oder in Prüfungsphasen seien weniger Menschen aktiv. Dies schränke die Möglichkeiten für Projekte ein (Int3:263; Int4:308; Int6:330,339). In den Greenpeace-Interviews wurde noch angemerkt, dass es problematisch sei, wenn Leute nicht regelmäßig teilnähmen (Int3:263,266), Konflikte durch ein unterschiedliches Verständnis von Mitwirkung entständen (Int4:489) oder Mitglieder nicht mehr kämen, wenn sie sich übergangen fühlten (Int4:526).

Generell sei oft zu wenig Rückmeldung vorhanden, um auf die Gründe für ein Aussteigen schließen zu können. So berichtete ein Mitglied des DJN:

„[...] wobei man halt auch dann nie so genau weiß, woran es lag zum Beispiel, wenn jemand die Gruppe verlässt, ob das einfach ein zeitliches Problem ist, ein Interessenproblem ist oder oder das halt an dem in der Gruppe liegt, man bekommt da halt keinen Feedbackbogen [...]." (Int1:276-278)

In einem der Interviews stellte sich heraus, dass der Umstieg von der Jugendarbeitsgruppe in die Erwachsenengruppe von Greenpeace sich häufig problematisch sei:

„Ich denke, das sind primär die Aktionsformen und das rumsitzen und planen, was man ja eh den ganzen Tag in der Uni macht und dann muss man das nicht abends auch noch freiwillig tun. Das ist auch einfach ein ganz anderes JAG-Gefühl, als ein Gruppengefühl, weil irgendwann mal irgendwie das Motto der JAGs war, Wir sind kreativ, frei und wild' oder so und die Gruppen sind vielleicht eher informativ, basisdemokratisch [...]." (Int5:231-236) 
Mit dem Alter änderten sich auch die Arbeitsweisen und Erwartungen an die Zusammenarbeit. Das beeinflusse die Aktionsformen und Strategien. Auch wenn es gewisse Voraussetzungen gebe, laufe Partizipation in jeder Untergruppe von Greenpeace anders ab. Langfristig funktioniere Partizipation nur, wenn sich Erwartungen und Arbeitsweisen deckten. Die Form des Plenums und die Aktionsformen der Erwachsenengruppe schrecke viele beim Eintritt aus der Jugendgruppe ab (Int5:233,346). Nach Aussage der Interviewten werde dieses Problem an übergeordneter Stelle in Hamburg diskutiert (Int5:240).

Festhalten lässt sich, dass Zeit ein wichtiger Faktor für Partizipation ist und dies auf zweierlei Weise: Zum einen ist die verfügbare Zeit eine Voraussetzung für die individuelle Teilnahme und zum anderen wird Zeit benötigt, um neue Mitglieder zu finden und einzubeziehen. Das häufig unbegründete Verlassen der Organisationen macht es schwierig, die Gründe für mangelnde Partizipation einschätzen zu können. Dennoch scheint es eine grundsätzliche Übereinstimmung von Arbeitsformen und Erwartungen innerhalb der Gruppe geben zu müssen, damit eine langfristige Partizipation stattfinden kann. Dies ist sicherlich ein wichtiger Aspekt, warum Menschen unterschiedliche Umweltorganisationen wählen.

\section{Förderlich für Partizipation}

In den Interviews wurden einige Vorschläge angeführt, damit Partizipation besser gelingen kann. Von Seiten des DJN wurde dahingehend angemerkt, dass häufige Treffen (Int6:444), eine kleinräumige Struktur, ohne große Entfernungen zu den Treffen (Int6:152,447) und eine verbesserte Betreuung für den Nachwuchs (Int6:134) sich positiv auf die Beteiligung auswirken würden. Die Betreuung und Einarbeitungszeit wurde auch bei Greenpeace erwähnt:

„Erstmal ist wichtig, dass alle miteinander kommunizieren können. Kommunikation ist das $\mathrm{A}$ und $\mathrm{O}$ und das ist essentiell wichtig. Und die Leute müssen wissen, wer, an wen sie sich wenden können. Und von daher krankt es oftmals, dass im Prinzip manchmal nicht ganz klar ist ,An wen kann ich mich wenden? Wie ist das aufgebaut? Wie ist die Struktur?' Es würde verbessert, wenn ich noch mehr Einarbeitungszeit investiert würde, dass die Leute noch mehr darüber wissen." (Int4:550-554)

Das heißt, Fragen stellen, eine längere Einarbeitungszeit und eine gute Kommunikation werden als förderlich für Partizipation angesehen. Dabei sei das Fördern von eigenverantwortlichem Arbeiten und das "Anfangen lassen“ wichtig (Int4:116,558). So „findet man für jeden irgendwie eine Aufgabe oder einen Platz" (Int4:533), da es viele verschiedene Aufgaben gebe (z.B. Recherchen, Gespräche mit PolitikerInnen, Presseerklärungen verfassen). Personen noch stärker in „kleine Aufgaben“ einzubinden, könne dabei helfen die Motivation zu erhöhen (Int5:463). Weitere Vorschläge 
waren, das Plenum interessanter und kreativer zu gestalten (Int5:445), mehr thematisch zu arbeiten (Int5:452) und neue Mitglieder stärker im Plenum einzubeziehen: „Und, wenn jetzt neue Leute hier reinkommen, dann kriegen die mit, was gemacht wird, aber sie kriegen nicht mit, warum es gemacht wird." (Int5:456-458). Außerdem wären eine größere Bekanntheit (Int4:565), eine langfristige Beteiligung der Mitglieder (Int4:306) und gruppendynamische Treffen (Int5:483) förderlich. Ein weiterer interessanter Aspekt ist die Zahl der Vereine, in denen sich Mitglieder betätigen:

„Das ist auch so ein Phänomen übrigens, was Umweltgruppen angeht: Wenn man dann einmal in einer drin ist, dann gibt es Leute, die sind halt in dieser Gruppe drin und machen dann ganz ganz ganz ganz schrecklich viel und dann gibt es Leute, die können sich nicht entscheiden. Die sind dann in acht Vereinen gleichzeitig und tauchen überall alle acht Wochen auf, weil das Plenum liegt immer gleichzeitig." (Int5:403-408)

Dies habe Vor- und Nachteile für die einzelnen Gruppen. Zum einen verfügten diese Personen oft über ein größeres thematisches und organisatorisches Wissen, brächten sich aber auch weniger in die einzelnen Gruppen ein (Int5:424,431). Zum anderen könnten dadurch neue Impulse in die Gruppen einfließen (Int3:383). Ein weiterer Aspekt ist das Finden und Stärken von eigenständig arbeitenden Mitgliedern:

„Interessantes Stichwort ist da eigentlich ,Macher machen'. Leute zu finden, die nicht nur mitlaufen, sondern wirklich eigenständig sagen: ,Das will ich machen! Das ist cool! Da stehe ich dahinter! Das will ich ausprobieren! Da möchte ich was erleben! Da möchte ich mich selber kennenlernen!' Und dann die Leute dahingehend zu motivieren, genau diese Aufgaben dann nachher auch durchzuführen." (Int4: 112-116)

Das Zitat zeigt, dass Greenpeace versucht Personen zu finden, die motiviert sind, Verantwortung zu übernehmen. Die Begriffswahl impliziert jedoch auch eine Form von Gruppenleitung.

Förderlich für die Partizipation ist zunächst eine kurze räumliche Entfernung zum Verein. Wie sich auch im Abschnitt über die Motivation gezeigt hat (siehe 5.2.2 , werden mangelnde Zeit und große Entfernungen häufiger als Grund angeführt, die gegen eine Teilnahme sprechen. In beiden Organisationen wird die Betreuung für EinsteigerInnen hervorgehoben. Erste Fragen können beantwortet werden und es fällt leichter, sich in die Strukturen einzufinden. Die Betreuung ist sogleich ein Teilaspekt einer funktionierenden Kommunikation: Aus den Interviews ließ sich entnehmen, dass ein guter Austausch über Verantwortlichkeiten und ein konstruktiver Umgang mit Konflikten für eine gute Arbeitsatmosphäre sorge. Die Zahl der Vereine, in denen Mitglieder sich engagieren, kann zusätzliche positive Effekte für die einzelnen Teilnehmenden und für die Gruppe haben, allerdings manchmal auch hinderlich sein. 


\title{
5.2.4 Auswirkungen des Engagements
}

Um mehr über die Motive und Ziele des Engagements zu erfahren und eine Einschätzung der persönlichen Entwicklung durch das Engagement zu erhalten, wurden die InterviewpartnerInnen gebeten die Auswirkungen ihres Engagements darzustellen. Sowohl für sich selbst, als auch für andere. Dieser Punkt lässt sich nicht eindeutig von dem Abschnitt Motivation (5.2.2) dieser Arbeit abgrenzen. Der Schwerpunkt in diesem Abschnitt liegt in der Einschätzung der eigenen Situation im zeitlichen Verlauf der Mitarbeit.

\section{Für sich selbst}

Im DJN wurde stark die Auseinandersetzung mit Natur hervorgehoben, insbesondere durch individuelle Erfahrungen:

\begin{abstract}
„Also, aber ich glaube, es hat schon irgendwie geholfen sich damit noch auseinander zu setzten und irgendwie selber zu merken warum, die Natur kennenzulernen, einfach selber draußen zu sein und irgendwie das auch zu schätzen [...]." (Int6:503-505)
\end{abstract}

Weiter hoben die Mitglieder des DJN hervor, dass sie durch ihre Mitarbeit im Verband die Fähigkeit erlernt oder weiterentwickelt hätten, mit anderen zusammenzuarbeiten und zu organisieren (z.B. Seminare, Exkursionen) (Int1:212; Int6:387). Die persönliche Weiterentwicklung tauchte in mehreren Interviews, sowohl bei Greenpeace, als auch im DJN auf (Int1:70; Int4:441), allerdings wurde im DJN besonders die Stärkung des Bewusstseins, der Selbstständigkeit und des Mutes hervorgehoben (Int1:204; Int6:509). Das folgende Zitat betont dabei die Weiterentwicklung von sich selbst und anderen durch die Arbeit im Verband:

„[... ] beim DJN hab ich so eher das Ziel, also das bewirkt gesehen, also, wenn ich ein Seminar organisiere sehe ich die Leute, die dann irgendwie fröhlich über die Wiese tanzen und wenn ich dann sehe, dass die Leute naturkundlich mehr Ahnung kriegen, macht das auch Sinn, also sich weiterzuentwickeln in der Arbeit." (Int1:66-70)

Bei den Interviewten von Greenpeace wurden insbesondere das positive Gefühl hervorgehoben, etwas aktiv zu verändern, Verbesserungen im Umweltschutz zu erreichen oder etwas „zu bewegen“ (Int3:229; Int4:439; Int5:376). Darüber hinaus spielten Spaß mit den anderen Mitgliedern und an den Themen, neue Herausforderungen und neue Kontakte eine Rolle (Int1:445,449,452; Int5:370). Das Sammeln von neuen Erfahrungen wurde von den InterviewpartnerInnen betont (Int1:195; Int4:444; Int6:387). 


\section{Für andere}

Bei den Auswirkungen des Engagements für andere wurden von Mitgliedern des DJN der Nutzen für den Verein hervorgehoben, dies beträfe z.B. die anstehenden Aufgaben, wie das Versenden von Materialien und die Suche nach neuen Mitgliedern und deren Einbindung (Int1:193; Int6:361). Außerdem wurde die Persönlichkeitsentwicklung und das Erlernen von fachlichem Wissen hervorgehoben (Int1:199), wie sich in dem folgenden Zitat zeigt:

„Es gibt viele Leute, die schon ab zwölf dabei waren und jetzt 19 sind, und manchmal sitzt man dann schon und denkt, Wow, die sind ja irgendwie schon relativ .. relativ krass selbstständig und mutig.' Wo ich immer denke, so war ich nicht, als ich 19 war." (Int1:202-205)

Wie schon unter Motivation (5.2.2) beschrieben, wird in den Interviews die positive Wirkung für den Naturschutz angeführt, die durch das Engagement erzielt werde (z.B. Int1:188,191; Int3:207,318; Int4:24; Int6:454). Bei Greenpeace wurden das Begeistern und Informieren der Öffentlichkeit für die Arbeit und die Themen und die Wirkung als Vorbild hervorgehoben (Int4:417,420,422). Sehr häufig genannt wurden bei Greenpeace die gesellschaftlichen Veränderungen, die durch die Arbeit erreicht werden könnten und die Möglichkeit, andere durch das Engagement zum Nachdenken und Handeln anzuregen (Int3:210; Int4:425,430; Int5:359,376).

„Zurückblickend hat es anderen, glaube ich, eine ganze Menge gebracht, dass ich viele Kühlschränke reformiert habe, ganz viele Stromanbieterwechsel, hoffentlich, angeregt hab und ja, es macht halt viele Leute aufmerksam auf Themen, also, in egal welcher Form man irgendwelcher Form erzählt, dass man halt bei Greenpeace ist oder Sachen macht, wird dann natürlich schon etwas thematisch rübergebracht." (Int5:355-359)

Vergleicht man die Einstellungen zum Engagement von Greenpeace und DJN, zeigt sich, dass im DJN eher die individuelle Ebene im Vordergrund steht. Es geht stärker darum, durch Erfahrungen in der Organisation und in der Auseinandersetzung mit Natur zu lernen und Wissen und Fähigkeiten weiterzugeben. Die Interviews zeigten, dass bei Greenpeace stärkeres Gewicht auf den gesellschaftlichen und politischen Einfluss in Umweltfragen gelegt wird und dass der Kontakt zu neuen Leuten und neue Herausforderungen eher im Vordergrund stehen. Die Strategie von Greenpeace, auf Außenwirkung zu setzen, findet sich in den Interviews wieder. Der DJN könnte in seiner Arbeit stärker als „nach innen gerichtet“, z.B. hinsichtlich der Weitergabe von Erfahrungen an Jüngere, beschrieben werden. Daraus ergeben sich verschiedene Zielgruppen an Mitgliedern für die Organisationen: Greenpeace zieht stärker Menschen an, die aktivistisch arbeiten wollen, wohingegen im DJN das Lernen über und das Erfahren von Natur im Vordergrund steht. 


\subsubsection{Zusammenarbeit}

In diesen Abschnitt wird die konkrete Zusammenarbeit in den Umweltorganisationen thematisiert. Zunächst wird darauf eingegangen, wie Entscheidungen in den Gruppen getroffen werden, wie Themen entwickelt werden und wie Projekte umgesetzt werden. Die Bedeutung der Altersstruktur und ihre Wirkung für die Zusammenarbeit werden in einem eigenen Abschnitt behandelt (5.2.6).

\section{Treffen von Entscheidungen}

Das Treffen von Entscheidungen ist ein wichtiger Aspekt in der Zusammenarbeit in Gruppen. Es wird über Ziele und Strategien entschieden, über die Auswahl von Themen, die Umsetzung von Projekten oder den Umgang mit Konflikten. Innerhalb des DJNs wird das Treffen von Entscheidungen auf Ebene der Ortsgruppen als „,basisdemokratisch“ angesehen (Int1:151). Auf Bundesebene wird dies von einer Interviewten folgendermaßen beschrieben 5 :

„Auf Bundesebene gab es dann .. kommt ja immer auf die Entscheidung drauf an. Auf Bundesebene gab es ja die Jahreshauptversammlung, wo dann entschieden, also, diskutiert wurde einfach und dann abgestimmt. Und wir haben kein basisdemokratisches System. Schon so, dass alle etwas sagen dürfen, aber nicht, dass man sich totdiskutiert, bis alle zufrieden sind [...].“ (Int6:206-209)

Hier zeigt sich, dass die Zusammenarbeit auf unterschiedlichen Ebenen abläuft. Es gibt eine Gruppenebene, auf der konkrete Entscheidungen für regionale Projekte getroffen werden und eine Bundesebene, auf der übergeordnete Ziele und Projekte besprochen und beschlossen werden. Die Form der Abstimmung auf Bundesebene, insbesondere auf der Jahreshauptversammlung, wird kritisiert: „die drei Alten diskutieren und entscheiden irgendwas und die ganzen Kleinen wissen nicht, worum es geht und heben ihre Hand oder lassen es sein“ (Int6:212-213). Das habe zur Folge, dass sich Jüngere manchmal unterordneten und sich teilweise eine autoritäre Struktur ausgebildet habe (Int6:204-206). Über Entscheidungen auf der Gruppenebene berichtete einer der Interviewten:

„Eigentlich wird jedes genommen, also jede Idee, aber ja einen richtigen Bestimmer gibt es bei uns eben nicht, das ist ja eben unser Motto, dass sobald man 25 ist, dass dann dass man dann eben rausgeschmissen wird sozusagen, ausgesockt, und dadurch gibt es dann keinen richtigen Bestimmer der sagt, das machen wir, das nicht', sondern jeder kann seine

\footnotetext{
${ }^{5}$ Die Interviewte hat den DJN bereits vor wenigen Jahren aufgrund der Altersbeschränkung verlassen und spiegelt nicht unbedingt die aktuelle Situation wieder.
} 
Seminare führen, seine Exkursionen machen und wird dann halt sehen, ob wer kommt oder nicht." (Int2:76-81)

Zusammengefasst gibt es Unterschiede im Entscheidungsverhalten zwischen Gruppenebene und Bundesebene. Auf Bundesebene müssen dabei mehr Personen berücksichtigt werden und es gibt Regelungen für die Abstimmungen, die sich in der Vereinssatzung finden (DJN 2010).

Bei Greenpeace werden die Entscheidungen eher als „demokratisch“ bezeichnet (Int3:86; Int5:153). Das wurde folgendermaßen beschrieben:

„Und wenn dann halt so mehrheitlich entschieden wurde, oder wenn da jetzt raus kam, dass niemand dagegen ist, weil das kann er dann ja sagen, dann wird das gemacht. Also, wir machen jetzt nicht ,Hat jemand was dagegen, dass wir jetzt diese Aktion machen?', weil jeder kann ja was sagen und dann machen wir das halt nicht mehr [...]." (Int5:149-153)

Hier wird viel Gewicht auf die Eigeninitiative der Mitglieder bei den Entscheidungen gelegt. Dies funktioniere nur dann langfristig, wenn Mitglieder eigeninitiativ handelten. Die Beeinflussung der Entscheidungen durch Ältere ist auch bei Greenpeace ein Thema:

„Das wird in der Gruppe entschieden, genau. Wird dann pro-contramäßig. Es gibt halt immer ein paar, die mehr Erfahrung haben als anderen, die dann eher die Entscheidung beeinflussen, weil sie schon wissen, wenn wir das da machen, ist es besser, aber eigentlich demokratisch, ja." (Int3:91-94)

Dabei ist allerdings immer zu berücksichtigen, wie konkret Einfluss auf die Entscheidungsfindung ausgeübt wird oder ob beispielsweise nur zusätzliche Informationen und Erfahrungen eingebracht werden. Die Gruppen haben eine gewisse Eigenständigkeit, aber es gebe relativ „strikte“ Vorgaben aus der Greenpeace Zentrale, wenn es um die Umsetzung von überregionalen Aktionen gehe (Int3:373). Da es sich bei Greenpeace um eine sehr große Organisation handelt, die klar strukturiert ist und über hauptamtliche Mitglieder in der Zentrale verfügt, ist der Einfluss der einzelnen Gruppen auf die gesamte Organisation eher als gering einzuschätzen (siehe auch 5.1.1).

Es zeigen sich drei Aspekte, die bei dem Treffen von Entscheidungen eine größere Rolle zu spielen scheinen: zum einen die Unterscheidungen zwischen demokratischen und basisdemokratischen Entscheidungen und zum anderen scheint ein größerer Einfluss von Älteren auf die Entscheidungsfindung zu bestehen. Drittens gibt es Unterschiede zwischen der Ebene der Ortsgruppen und der Bundesebene. Diese zeigen sich deutlich in beiden Organisationen. Da es sich bei Greenpeace 
um eine sehr große, relativ hierarchisch organisierte Struktur handelt, sind die individuellen Einflussmöglichkeiten geringer. Der DJN ist im Vergleich nicht so stark strukturiert und verfügt über weniger Mitglieder, so dass Einzelne eher die Möglichkeit haben, sich auf Bundesebene einzubringen.

\section{Auswahl der Themen}

Durch die Auswahl von Themen und die Strategien ihrer Umsetzung können Umweltorganisationen ihre eigenen Schwerpunkte setzen. Bis zu einem gewissen Grad sind Themen durch Satzungen oder allgemeine Strukturen (z.B. durch die Zentrale bei Greenpeace) vorgegeben, aber es gibt einen gewissen Interpretations- und Gestaltungsspielraum. Wie im Kapitel zu den Umweltbewegungen beschrieben (siehe Kapitel 2), ändern sich Themen und Schwerpunkte im Laufe der Zeit.

Die Themen von Greenpeace sind stark durch allgemeine deutschlandweite und internationale Strategien bestimmt. Zu den Schwerpunktthemen (siehe 5.1.1) gibt es feste AnsprechpartnerInnen, wie eine der Interviewten beschrieb:

„Ja, also es gibt ja verschiedene Aktionsansprechpartner in der Gruppe. [...] meistens ist es so, dass der Ansprechpartner für das Thema sich um die Organisation kümmert, dass die Materialien da sind und den Treffpunkt und sowas und dann macht man halt die Aktion." (Int3:5666)

Dies scheint im Allgemeinen das Vorgehen bei der Themenwahl zu sein: Die ThemenansprechpartnerInnen bekommen Vorschläge von der Zentrale und diese werden in die Gruppe getragen, besprochen und es wird diskutiert, ob und wie diese umgesetzt werden können (Int3:65; Int4:63,66,79). Allerdings gebe es auch Themen, die aus der Gruppe heraus entständen (Int3:67; Int4:153) und worüber die Gruppe eigene Schwerpunkte setzen könne (Int3:378). Nach Aussage eines Interviewpartners habe die Göttinger Gruppe wenige eigene und langfristige Themen, da es durch die vielen Studierenden in der Gruppe zu häufigen Wechseln komme (Int4:296,664). Nach einer Vorstellung der Themen im Plenum, werde deren Umsetzbarkeit anhand der verfügbaren Zeit, der Kapazitäten, der Zahl der Beteiligten und dem organisatorischen Aufwand geprüft (Int4:68,72), denn „,[w]enn keine Ressourcen da sind, dann kann das Thema noch so spannend sein, dann brauchen wir das gar nicht angehen." (Int4: 157-159).

Die Vorgaben von Greenpeace Deutschland haben den Effekt, dass zum einen weniger eigene Themen gesucht werden müssen, auch wenn dies zu mehr Unabhängigkeit und Eigenständigkeit der Gruppen führen könnte (siehe 5.2.2). Auf der anderen Seite stehen die vorgegebenen Aktionen in einem größeren Zusammenhang, sind gut umsetzbar und Informationsmaterial wird zur Verfügung gestellt. 
Der DJN sei stärker auf die Initiative Einzelner angewiesen, die durch eigenes Engagement Themen einbrächten (Int1:30,106). Dies geschehe zumeist durch persönliches Interesse an den Themen oder durch Empfehlungen (z.B. die Sichtung seltener Vögel) (Int1:89). Auf die Frage, wie Ideen eingebracht werden, sagte einer der Interviewpartner: „Das gilt eigentlich für jeden, jeder gibt.“ (Int2:66). Der DJN ist in diesem Sinne darauf angewiesen, dass aktiv Themen eingebracht werden. Veranstaltungen, wie regelmäßige Exkursionen oder Seminare, entstehen aus Eigeninitiative.

Die Möglichkeit, persönliche Themenwünsche einzubringen, wird von Interviewten beider Organisationen gesehen (Int1:113; Int4:83,142). Das Festlegen von Themen geschieht in beiden Organisationen unterschiedlich: Während bei Greenpeace überwiegend Vorgaben von der Zentrale gemacht werden, die umgesetzt oder modifiziert werden können, ist der DJN auf selbstständig entwickelte und eingebrachte Themen angewiesen.

\section{Umsetzung von Projekten}

Aus den Ideen und Themen entwickeln sich Projekte. Die Umsetzung dieser Projekte erfolgt auf unterschiedliche Art und Weise. Hier spielen die Strategien und Ziele der Organisationen eine Rolle.

Im DJN fänden sich für Projekte so genannte Orga-Teams zusammen, welche die Verantwortung für die Durchführung übernähmen (Int1:119). Die Arbeit der Orga-Teams beschrieb einer der Interviewpartner:

„Das findet sich auch quasi durch Interesse oder durch Freundschaftsbande oder so und das kann auch unterschiedlich groß sein, also es gibt Leute, die organisieren ein Seminar alleine oder auch ...etwas kombinierte Teams, mit wer schon etwas erfahren ist und wer der oder die lernen möchte, wie man das Seminar organisiert, was man dabei bedenken muss, Verpflegung, Unterkunft und so, genau. Meistens ist es aber relativ klein, so zwei, drei Leute, bei diesen Wochenend-Seminaren." (Int1:122127)

Bei der Umsetzung der Projekte zeigen sich wieder Motive, die in den Interviews mit dem DJN häufiger auftauchten. In diesem Fall wird der gegenseitige Austausch von Erfahrungen genannt, der in Teams mit gemischtem Alter oder unterschiedlicher Erfahrung stattfinde. Ein persönliches Interesse motiviert dazu, eigenständig Themen einzubringen. Der Begriff „Freundschaftsbande“ zeigt, dass der Umgang miteinander nicht nur auf die Projekte oder den Verein beschränkt ist. Dies wird auch an anderer Stelle betont: „weil man die Leute mehr kennt und sich jede Woche sieht quasi Gruppenarbeit" (Int1:96). Problematisch sei es, wenn die Verantwortlichkeiten 
für bestimmte Aufgaben nicht geklärt seien. Dies habe insbesondere einen negativen Einfluss auf die Zusammenarbeit mit externen Vereinen (Int1:165).

Bei Greenpeace werden die Aktionen über das Plenum oder intern über das Internet organisiert (Int3:128). Als wichtig für eine gelungene Aktion wurden dabei die Zuverlässigkeit der Personen und günstige Rahmenbedingungen (z.B. Ort, Wetter) genannt (Int4:188). Ausschlaggebend sei, dass „man wirklich dann als Team zusammenarbeitet" (Int4:390), wobei die Gruppenzusammensetzung über den Erfolg von Projekten bestimme (Int3:70). Die zeitlichen Ressourcen seien ein wichtiger Faktor für die Projektumsetzung. Es hänge davon ab, ob sich genügend Personen fänden, die Zeit hätten, da sonst die Projekte scheiterten (Int4:194; Int5:142). Insbesondere bei parallel verlaufenden Projekten sie dies problematisch (Int5:141). In allen Greenpeace-Interviews erschien die Verantwortlichkeit für die Projektumsetzung besonders wichtig: Wenn es keine Verantwortlichen oder AnsprechpartnerInnen für Projekte gebe, bzw. diese wegfielen, könne es zum Scheitern eines Projektes kommen (Int3:139,145; Int4:132; Int5:178). Eine Interviewte beschrieb ein Beispiel:

„Die andere Möglichkeit ist, dass eine Idee in der Gruppe entsteht, so etwas wie die Papier-AG, da ist meistens das Problem, es fühlen sich alle so ein bisschen angesprochen, aber keiner so richtig und deshalb läuft das meistens etwas chaotischer ab und ja, aber ... kommt immer darauf an, wer sich da findet, ob das dann Erfolg hat oder nicht." (Int3: 66-70)

Für die Umsetzung von Projekten scheint die Verantwortung von grundlegender Bedeutung zu sein. Diese ist bei der Arbeit mit Freiwilligen besonders wichtig und kann über Erfolg und Misserfolg von Projekten entscheiden. Das „Zeitproblem“ scheint bei dem DJN nicht in dieser Weise aufzutauchen, da die Entscheidung für und die Umsetzung von Projekten stark über Eigeninitiative abläuft. Da es bei Greenpeace auch Vorgaben oder Vorschläge der Zentrale gibt, besteht die Entscheidung eher in der Frage nach der Umsetzbarkeit der Projekte. Das ist besonders von der verfügbaren Zeit und den Personen abhängig.

\section{Arbeit in der Gruppe}

In Bezug auf die Zusammenarbeit wurden in den Interviews noch Themen angesprochen, die sich nicht den dargestellten Kategorien zuordnen ließen, aber wichtig für die Thematik sind. Auf diese soll im Folgenden kurz eingegangen werden.

In einem der Interviews wurde betont, dass sich die Arbeitsweise in Unternehmen von der in Freiwilligenorganisationen unterscheide, dies sei z.B. an anderen Herangehensweisen durch Studierende im Gegensatz zu Angestellten zu beobachten (Int4:501,504). Ferner sei das Ausüben von Druck ineffektiv und führe zu einer geringeren Beteiligung (Int4:392). Dennoch müsse man sich 
„[...] in gewissen Dingen [...] schon durchsetzen , das muss jetzt mal gemacht werden als Thema', aber man muss dann mitziehen natürlich auch, man kann eben nicht andere machen lassen, das funktioniert nicht $[\ldots] . "($ Int4:516-518)

In dieser Aussage klingt eine Form von Gruppenleitung an. Hier zeigt sich, dass es wichtig sein kann einen Mittelweg zwischen dem Motivieren und Fordern von anderen und dem eigenen Engagement zu finden. Weiter wurde betont, dass stabile Strukturen wichtig seien, um Phasen mit geringer Aktivität, wie z.B. Semesterferien, zu überbrücken (Int5:495). Dennoch solle immer noch die Möglichkeit vorhanden sein, bestehende Hierarchien zu verändern (Int5:492). In einem GreenpeaceInterview wurde darauf hingewiesen, dass sich die Besetzung der Rollen (KoordinatorInnen, AnsprechpartnerInnen) im Team änderten und es wichtig sei, Verantwortung wieder abzugeben zu können (Int4:397,399,400). Um Problemen vorzubeugen und Projekte auszuwerten, gebe es Feedback-Runden und -Gespräche nach Aktionen oder bei Unzufriedenheiten oder Konflikten (Int3:151; Int4:156,180). In einem der DJN-Interviews wurde die Wichtigkeit betont, Rücksicht auf Jüngere zu nehmen und sich in ihre Situation hineinzuversetzen. Eine gute Zusammenarbeit kann sich sehr motivierend auswirken: „Und das war eben auch so ein kleines Aha-Erlebnis, wenn man dann so irgendwie in die Gemeinschaft rein findet und vor allen Dingen auch merkt, dass man was verändern kann und so." (Int3:174-176).

Neben der grundlegenden Strukturierung der Zusammenarbeit gibt es noch viele verschiedene Punkte, die in dieser Arbeit nicht näher behandelt werden können. Darunter fallen z.B. der Umgang mit Konflikten, das Einnehmen von Rollen in den Gruppen und die Auswirkungen von Stress auf das Engagement und die Zusammenarbeit. Diese Aspekte beeinflussen die anderen Aspekte, wie die Motivation oder die Auswahl von Themen, stehen dabei aber nicht im Vordergrund.

\subsubsection{Altersstruktur}

In Bezug auf die Altersstruktur unterscheiden sich die beiden Organisationen. Bei Greenpeace gibt es verschiedene Gruppen mit Altersklassen: Greenteams (zehn bis 15 Jahre), JAGs (15-19), Greenpeace-Gruppen (19-50) und Team50plus (älter als 50 Jahre) (Greenpeace 2010b). Hier steht eher der Übergang zwischen den Altersgruppen im Vordergrund. Im DJN gibt es eine klare Altersbegrenzung zwischen zwölf und 25 Jahren. Danach erlischt der Status als Mitglied. Im Folgenden wird hauptsächlich auf den DJN eingegangen, da die Altersbegrenzung ein entscheidendes Moment im Selbstverständnis der Organisation darstellt. Die häufigen Wechsel, die durch die Altersstruktur bedingt sind, werden im DJN als „ständiger Umbruchprozess“ wahrgenommen (Int1:229). Die Altersbegrenzung habe den 
„[...] Hintergrund, dass sich ja bei einer größeren Altersspanne schon eine Hierarchie ausbildet für die Älteren, Klügeren, Erfahreneren und dann die Sachen machen, die Jüngere dann konsumieren. Das hat man schon ganz bewusst eingerichtet. Und das hat sich auch erstaunlicherweise bis heute gehalten, obwohl es immer wieder so Versuche gibt, das aufzuweichen, grade wenn irgendwie wenig Leute dabei sind und es ist natürlich schade, wenn jetzt XY verliert nächstes, also verliert in Anführungsstrichen, nächstes Jahr [...]." (Int1:223-228)

Hier zeigt sich, dass die Vermeidung von Hierarchien ein ganz zentraler Punkt für die Einführung der Altersbegrenzung ist. Es ist als bewusste Strategie zu sehen, um zu vermeiden, dass sich autoritäre Strukturen oder zu große Erfahrungsasymmetrien ausbilden. Da der DJN 1950 gegründet wurde (DJN 2010) zeigt sich hier ein Gegenentwurf zu den damals bestehenden Verhältnissen. Insofern ist interessant, dass sich diese Struktur über 60 Jahre lang gehalten hat. Es hat jedoch den Anschein, als dass der Verein noch immer versucht, eine Alternative zu gesellschaftlichen Verhältnissen zu bieten (vergleiche auch 5.2.3). In dem Zitat klingt auch an, dass es sich bei dem Verlassen des Vereins mit 25 Jahren um einen bewussten Prozess der „Loslösung“ und des Lernens handelt. In einem weiteren Interview hieß es:

„Ja, ich glaube, die Jüngeren haben von den Älteren, dass ihnen der Rahmen geboten wird, dass die kommen können, Natur erleben und auch ein anderes gesellschaftliches Zusammensein vielleicht erleben und dass sie auch entscheiden dürfen, also ich weiß nicht, ich glaube, das ist nicht unbedingt immer der Fall, dass man ihnen offen lässt, was sie machen wollen und dass sie sich das überlegen müssen, dass sie, wenn sie wollen, auch mal nix tun." (Int6: 366-370)

Die Unterschiede in den Erfahrungen werden hier als Möglichkeit des gemeinsamen Lernens und der Weitergabe von Wissen gesehen. Die Alternative zu bestehenden gesellschaftlichen Verhältnissen wird auch hier explizit, im Sinne eines „anderen“ Verständnisses von Gesellschaft, angesprochen. Wie bereits im Abschnitt Partizipation 5.2.3 in diesem Kapitel dargestellt, ist die Entwicklung von Selbstständigkeit und Entscheidungsfreiheit bei Jüngeren ein wichtiges Motiv für die Arbeit im DJN. Das ist besonders in Abgrenzung zu einer Gesellschaftsvorstellung zu sehen, in der diese nicht als gegeben angesehen oder vermittelt wird. Ein Entwicklungsprozess bei den jüngeren Mitgliedern wird angesprochen: „dann merkt man schon, dass sie mittlerweile auch ordentlich mitmischen und am Anfang immer nur da standen." (Int6: 423-424). Das „Bestimmer“ (gemeint sind ältere Mitglieder) mit einem höheren Alter die Gruppe verließen, werde als sehr positiv wahrgenommen (Int2:81). Damit sei es möglich „Platz [...] für neue Ideen und für neue Leute“ zu machen und Jüngeren eine Möglichkeit zu geben, ,sich Gedanken zu machen und sich zu kümmern.“ (Int6: 
95-98). Kindern und Jugendlichen durch die Mitarbeit ein anderes Gesellschaftsbild zu vermitteln, ist somit eines der zentralen Ziele des DJN.

Bei Greenpeace gibt es eine vorgegebene Teilung in Altersgruppen. Die Altersunterschiede innerhalb dieser Gruppen wurden positiv wahrgenommen:

„[...] die Mischung finde ich eigentlich ganz gut, weil die Älteren bringen dann immer noch mehr Informationen rein und die jüngeren haben dann halt mal mit ,Warum können wir das dann nicht machen?' so, und von daher finde ich das eine ganz gute Mischung so. Ich glaube ich fände es eher schade, wenn es jetzt nur eine Altersgruppe wäre." (Int3: 196-201)

Dennoch kann der Übergang zwischen den verschiedenen Greenpeace-Gruppen problematisch sein, wie schon unter 5.2 .3 bereits angemerkt. Es wurde darauf hingewiesen, dass die Arbeitsweisen im Plenum und die Aktionsformen zwischen der JAG und der Erwachsenengruppe z.T. sehr unterschiedlich sei und dass dadurch viele der Jüngeren aus den JAGs nicht langfristig in den Erwachsenengruppen bleiben wollten (Int5:19). So werde z.B. eine geringere Kreativität, weniger inhaltliche Diskussionen in den Erwachsenengruppen und ein „anderes Gruppengefühl" in den JAGs hervorgehoben (Int5:27,33,236).

Das Alter ist somit ein wichtiges Element für die Zusammenarbeit in den Gruppen. In beiden Gruppen gibt es einen altersabhängigen „Bruch“, entweder durch das Ausscheiden aus dem Verein oder durch den Wechsel in eine andere Gruppe. Die Vorteile einer Zusammenarbeit in Gruppen mit verschiedenem Alter wurden in beiden Umweltorganisationen betont.

\subsubsection{Bedeutung des Internets}

Besonders in den Greenpeace-Interviews zeigt sich, dass der Austausch über das Internet eine immer größere Rolle für die Koordination und Zusammenarbeit spielt. Eine Interviewte berichtete:

„Neuerdings läuft auch viel über den Airport. [...] Das ist ein Internetportal, intern, für Greenpeace, wo man nur aufgenommen wird, wenn man auch aktiv in der Gruppe ist, [...] es gibt auch so verschiedene Gruppen zu verschiedenen Aktionen oder zu verschiedenen Themen und darüber läuft jetzt seit neusten, seit das einigermaßen gut funktioniert auch ziemlich viel." (Int3:110-116)

Über den Airport werden Erfahrungen auch zwischen Greenpeace-Gruppen aus verschieden Städten ausgetauscht, was besonders für die Organisation von deutschlandweiten Gruppenaktionstagen hilfreich sei (Int3:127; Int5:164). In Göttingen fände, ergänzend zum Plenum, ein Austausch durch verschiedene Medien wie Email, 
SMS und den Airport statt (Int5:115) und es gebe einen Newsletter (Int5:522). Die Verbindung zur Greenpeace Zentrale laufe hauptsächlich über Email, in dringenden Fällen werden die ThemenansprechpartnerInnen direkt angerufen (Int4:77,84). Allerdings komme es bei der Internetnutzung stark darauf an, „wie sehr man sich da selber reinhängt“ (Int3:121). Insofern hängt die Nutzung von Internettechnologien von der Eigeninitiative der AnwenderInnen ab. Über die Gruppenebene hinaus, sei ein „neuer Trend“ (Int5:592) zur Internetnutzung zu erkennen und es laufe „unglaublich viel mehr digital“ ab (Int5:121). Eine der Interviewten fasste die Entwicklungen zusammen:

„Ja, übers Internet wird viel mehr gemacht. Eigentlich läuft ganz viel über Twitter und Facebook und alles, die ganzen Aktionen oder viele Aktionen werden gefilmt und auf YouTube hochgestellt. Fotos werden veröffentlicht im Internet, es wird ganz viel in Foren geschrieben. Also, grade von Holland aus, wird ganz viel gemacht, von Amsterdam aus, die sitzen den ganzen Tag soweit ich weiß nur da und schreiben in Foren. Ich habe in Kopenhagen Leute getroffen, die das hauptberuflich machen und die sagten, es gebe einen Boom an Foren, einen Boom an Sachen, die man alles nur übers Internet publizieren muss. Und richtig stellen $[\ldots] . *\left(\right.$ Int5:583-589) ${ }^{6}$

Wie schon in den Darstellungen der Organisationen in diesem Teil (siehe 5.1) angemerkt, hat die Medienwirksamkeit einen sehr hohen Stellenwert bei Greenpeace. In den Interviews zeigt sich, dass dabei zunehmend Technologien des Web-2.0 (wie soziale Netzwerke, Videoplattformen) eingesetzt werden, um Bilder, Videos und Informationen zu verbreiten, neue Mitglieder zu finden und bestehende Strukturen besser zu vernetzen. Umweltorganisationen sind an aktuellen Trends (wie z.B. social media) interessiert und wissen neue Technologien für sich zu nutzen, die z.B. im Journalismus oder in Unternehmen zunehmend verwendet werden (SZ 2009, 2010; ZEIT 2010). Auf Konsequenzen, auch für die individuell Beteiligten, wurde an anderer Stelle bereits hingewiesen (siehe 5.2.1).

Der Einsatz von neuen Medien wurde in den Interviews mit dem DJN nicht erwähnt, lediglich in der Form von Absprachen über einen Email-Verteiler (Int2:58). Beide Organisationen verfügen über Webseiten, die regelmäßig aktualisiert werden (DJN 2010d, GREenPeace 2010a).

Es zeigt sich, dass insbesondere bei Greenpeace die Verwendung des Internets für die Planung und Wirksamkeit von Aktionen eine große Rolle spielt. Die Einbeziehung von sozialen Netzwerken im Internet scheint an Bedeutung zu gewinnen. Eine regelmäßig aktualisierte Website scheint selbstverständlich.

\footnotetext{
${ }^{6}$ Die Zentrale von Greenpeace International hat ihren Sitz in Amsterdam.
} 


\subsubsection{Austausch zwischen Gruppen}

Zwischen den Göttinger Umweltorganisationen bestehen Kontakte die mehr oder weniger intensiv sind. In Göttingen haben die meisten Organisationen ihr Büro im Göttinger Umwelt- und Naturschutzzentrum (GUNZ), einem e.V. der sich aus verschiedenen Organisationen zusammengeschlossen hat, um „Synergie-Effekte“ zu nutzen (GUNZ 2010). Greenpeace und der DJN sind Mitglieder im GUNZ.

Der DJN pflegt einen Informationsaustausch mit anderen Gruppen und arbeitet teilweise mit ihnen zusammen, beispielsweise „wo es halt ein Verein nicht alleine organisieren kann oder es auch spannender ist" (Int1:317-318). Außerdem gibt es Kontakte zu anderen Göttinger Einrichtungen, wie der Volkshochschule, mit der z.B. Vortragsreihen organisiert werden (Int1:321). Ein wissenschaftlicher Kontakt entstehe zumeist über Studierende (Int1:334).

Etwas anders stellt es sich bei Greenpeace dar: Zwar gebe es viel Austausch zwischen Greenpeace-Gruppen (Int3:110), aber Greenpeace sei „sehr vorsichtig“ bei der Zusammenarbeit mit externen Gruppen (Int4:251). Zwei Gründe wurden dafür hervorgehoben: zum einen solle die Unabhängigkeit gewahrt bleiben (Int4:217) und zum anderen sei es häufig problematisch für andere Gruppen, da man „anders wahrgenommen werde“ (Int4:225) und ,andere Organisationen, grade kleinere, gehen dann gerne mal unter" (Int4:220). Die Bekanntheit und das Image von Greenpeace stellen sich somit teilweise als hinderlich für Kooperationen heraus. Generell gebe es nur wenige Kooperationen. Bei Aktionstagen oder Ähnlichem seien mehrere Organisationen präsent (Int3:338), aber z.B. gemeinsame Infostände gebe es nicht, um die Unabhängigkeit zu wahren (Int4:239).

Wenn die AnsprechpartnerInnen für externe Gruppen unklar seien oder fehlten, seien Kontakt und Zusammenarbeit mit diesen Gruppen häufig problematisch. Dies betonten sowohl Mitglieder des DJNs, als auch von Greenpeace (Int1:162,167; Int4:553).

Der Austausch mit anderen Gruppen wird von beiden Organisationen betrieben. Hauptsächlich geht es dabei um den Austausch von Informationen und Kontakten, manchmal auch um gemeinsame Aktivitäten. Greenpeace ist in dieser Beziehung sehr vorsichtig, um die eigene Unabhängigkeit zu wahren und um zu vermeiden, dass sich das Image auf andere Gruppen überträgt. In beiden Organisationen wurde es als problematisch für die Zusammenarbeit gesehen, wenn keine AnsprechpartnerInnen für externe Gruppen vorhanden sind. 


\subsection{Zusammenfassung der Ergebnisse}

Im Folgenden werden kurz die wichtigsten Ergebnisse aus der Interviewauswertung zusammengefasst.

\section{Struktur}

Greenpeace ${ }^{7}$ ist eine große international agierende Umweltorganisation, das bedeutet auch, dass die Strukturen und Themen für die Ortsgruppen relativ stark vorgegeben sind. Es gibt Budgets für Aktivitäten. Der DJN ist eine eher kleine Organisation, in der eine größere Beteiligung der Mitglieder möglich ist. Themenwahl und Projekte sind auf eine hohe Eigeninitiative angewiesen.

\section{Motivation}

Der Zugang zu beiden Organisationen erfolgt meist über Freunde und Bekannte, wenn genügend Zeit für das Engagement zur Verfügung steht. Die Entscheidung für die Teilnahme entsteht meist bewusst und durch eigene Initiative. Interesse an den Themen Natur und Umwelt spielen für alle Interviewten eine Rolle, dies muss aber nicht der ausschlaggebende Aspekt sein. Auffällig ist, dass bei Greenpeace eher von „Umwelt" und im DJN eher von „Natur" gesprochen wird. Für die Mitglieder des DJN sind das Lernen und Vermitteln von Wissen und die persönlichen Beziehungen zu anderen eine große Motivation für die langfristige Mitarbeit. Bei Greenpeace wurde besonders das Bewirken von Veränderungen genannt.

\section{Partizipation}

Partizipation wurde meist mit „teilnehmen“ assoziiert. Der Schwerpunkt ist bei Greenpeace eher eine aktive Haltung einzunehmen und Ziele zu verfolgen. Im DJN sind die Übernahme von Verantwortung, mitgestalten zu können und die Abgrenzung zu anderen Vereinen und dem Alltag für das Verständnis von Partizipation vordergründig. Einig sind sich die Mitglieder beider Organisationen, dass Partizipation generell erlernbar ist, aber individuell unterschiedlich erlernt wird. Dies geschieht aktiv, also durch eigenes Engagement. Probleme in Zusammenhang mit Partizipation gibt es besonders durch eine geringe Beteiligung in Schul- und Semesterferien, wenn Personen nicht regelmäßig zu Treffen und Plena kommen oder durch geringe oder keine Rückmeldung, wenn Personen die Organisation verlassen. Im DJN erscheint das Finden von neuen Mitgliedern durch die Altersbegrenzung und die teilweise große Entscheidungsfreiheit schwierig. Generell sind eine kurze

\footnotetext{
${ }^{7}$ Um das Lesen zu erleichtern, wird hier nur von „Greenpeace“ oder dem „DJN“ gesprochen. Die Aussagen beziehen sich aber nur auf die Aussagen aus den Interviews.
} 
Entfernung zu den Gruppentreffen, eine gute Betreuung für EinsteigerInnen und eine funktionierende Kommunikation untereinander förderlich. Gruppendynamische Treffen wurden als Vorschlag angeführt.

\section{Engagement}

Die Auswirkungen des Engagements für sich und andere besteht beim DJN besonders daraus, etwas zu lernen, dieses Wissen anderen zu vermitteln und neue praktische Erfahrungen zu sammeln. Bei Greenpeace wurden besonders der gesellschaftliche Einfluss, das Schließen von neuen Bekanntschaften und neue Herausforderungen genannt. Insbesondere in den Aussagen zum Engagement wurde deutlich, dass die beiden Umweltorganisationen unterschiedliche Zielgruppen an Mitgliedern ansprechen.

\section{Zusammenarbeit}

Es gibt Unterschiede zwischen Entscheidungen auf übergeordneter Ebene und Gruppenebene. Bei Greenpeace gibt es auf übergeordneter Ebene kaum Möglichkeiten mit zu entscheiden, da die Organisation verhältnismäßig hierarchisch organisiert ist. Auf Gruppenebene wurden die Entscheidungen als „demokratisch“ bezeichnet. Der DJN bezeichnet sich eher als „basisdemokratisch“ auf Gruppenebene. Auf der Bundesebene werden mehrheitliche Entscheidungen getroffen. Der größere Einfluss von Älteren oder Erfahreneren auf die Entscheidungsfindung wird in beiden Gruppen gesehen. Hinsichtlich der Auswahl der Themen gibt es bei Greenpeace viele Vorgaben, auch wenn Spielräume bei der Umsetzung bestehen. Im DJN ist die Themenwahl stark auf die Eigeninitiative der Mitglieder angewiesen. Hinsichtlich der Umsetzung von Projekten ist für beide Organisationen zentral, dass sich Personen finden, welche die Verantwortung für ein Projekt übernehmen. Ob ein Projekt umgesetzt wird oder nicht, wird bei Greenpeace anhand der zur Verfügung stehenden Zeit und Ressourcen entschieden. Im DJN sind es eher die Eigeninitiative von Einzelnen oder einer Gruppe und das wie der Umsetzung entscheidend. Die Zusammenarbeit in den Gruppen wird noch von weiteren Aspekten beeinflusst, darunter der Umgang mit Druck, die Abgabe von Verantwortung, die Stabilität der Strukturen und der Umgang mit Konflikten.

\section{Alter}

Das Alter ist ein wichtiger Faktor in der Zusammenarbeit und in beiden Gruppen wurde ein gemischtes Alter als Vorteil bewertet. In beiden Organisationen gibt es altersbedingte „Brüche“: Bei Greenpeace durch Wechsel zwischen verschiedenen 
Greenpeace-Altersgruppen, die teilweise problematisch sind. Im DJN gehört die Altersbeschränkung von 25 Jahren zum Selbstverständnis der Organisation und soll zum Hierarchieabbau beitragen.

\section{Internet}

Für die Koordination und Zusammenarbeit erscheint das Internet immer wichtiger. Besonders Web-2.0-Technologien, Foren und soziale Netzwerke werden, insbesondere bei Greenpeace, zunehmenden für die Kommunikation eingesetzt. Bei Greenpeace ist der Einsatz des Internets ein entscheidender Teil, der auf Medienwirksamkeit setzenden Strategie. Beide Organisationen verfügen über Webseiten, die regelmäßig aktualisiert werden.

\section{Austausch mit anderen Gruppen}

Beide Organisationen tauschen sich mit anderen Göttinger Vereinen und Gruppen aus. Teilweise findet eine zeitlich begrenzte Zusammenarbeit zu bestimmten Themen statt. Greenpeace agiert dabei sehr vorsichtig, um die Unabhängigkeit nicht zu gefährden. Fehlende AnsprechpartnerInnen in der eigenen Gruppe können die Zusammenarbeit mit externen Gruppen erschweren. 


\section{Diskussion}

An dieser Stelle werden die untersuchten Umweltorganisationen zunächst in das Feld der Deutschen Umweltbewegungen- und organisationen eingeordnet. Danach werden die Themen Zivilgesellschaft und Partizipationstheorie (siehe Theoretischer Hinter-

grund (3.2) wieder aufgegriffen und mit den Erkenntnissen aus der empirischen Arbeit verglichen. Im Anschluss erfolgt die Diskussion der Forschungsfrage und der Hypothesen.

\subsection{Einordnung in Umweltbewegungen}

Die beiden Organisationen lassen sich in den allgemeinen Rahmen der Umweltbewegungen einordnen. Im Schema von KERN (2008) (siehe 2.5.5) schließt der DJN am ehesten an die Linie des „traditionellen Naturschutzes“ an, was sich insbesondere an dem Schwerpunkt des subjektiven Erlebens von Natur festmachen könnte. Die Themen Arten- und Naturschutz passen auch in dieses Feld. Allerdings sind die gesellschaftlichen Veränderungen, die angestrebt werden sollen, und die bewusste Abgrenzung zu hierarchischen Strukturen nicht unbedingt Elemente des „traditionellen Naturschutzes“. Durch das eher emanzipative Selbstverständnis des DJN sind Anknüpfungen zur frühen Umweltbewegungen erkennbar, die sich in den 1960er Jahren verstärkt in Deutschland zu etablieren begannen, und an Ideen aus der Wandervogelbewegung, die sich zu Beginn des 20. Jahrhunderts bildete (SAUR 2002). In den Interviews hat sich gezeigt, dass der Protest gegen Atomkraft ein relevantes Thema in den Anfängen des DJN war. Heute stehen andere Themen im Vordergrund.

Greenpeace lässt sich in KERNS (2008) Schema als „Internationale Organisation" bezeichnen. Auch Themen, die auf lokaler Ebene behandelt werden, sind in einen größeren internationalen Zusammenhang eingeordnet. Die Strategien haben eine internationale Ausrichtung und werden dann in regionalen Aktionen umgesetzt. Bei Greenpeace fällt auf, dass sich sowohl Interessengruppen-, als auch soziale Bewegungsstrategien, wie MARKHAM (2004) sie beschreibt (siehe 2.5.5), wiederfinden lassen: Das Spektrum reicht von Lobbyarbeit bis hin zum Aufbau von öffentlichem Druck auf Regierungen und Unternehmen.

Die allgemeinen Trends in den Umweltbewegungen lassen sich auch auf 
lokaler Ebene beobachten. Hier zeigt sich, dass sich die beiden Organisationen stark unterscheiden: Innerhalb von Greenpeace, als sehr große, international agierende Umweltorganisation, ist die zunehmende Professionalisierung ein Thema. Es gibt fest angestellte MitarbeiterInnen, die z.B. in der Zentrale in Hamburg arbeiten. Allerdings lässt sich auch ein Trend zur Professionalisierung in den lokalen Gruppen erkennen. Es gibt verschiedene Formen von Weiterbildungsveranstaltungen, bei denen das Spektrum von Koordinations-Workshops bis zu spezielleren fachlichen Seminaren reicht. Innerhalb der Gruppe gibt es feste Aufgabenverteilungen, im Falle der Göttinger Greenpeace-Gruppe z.B. einen Gruppenkoordinator, ThemenansprechpartnerInnen und einen Pressesprecher. Die Institutionalisierung lässt sich auch auf lokaler Ebene beobachten. Die Struktur von Greenpeace ist relativ stark durchgeplant, es gibt feste Gruppeneinteilungen nach Altersklassen und gewisse festgelegte Verantwortlichkeiten (z.B. für die finanzielle Planung). Materialien oder Kleidung, dass dem cooperate design von Greenpeace entspricht, werden zur Verfügung gestellt. Das passt zum Aufbau von Images: Die Überparteilichkeit und die Unabhängigkeit von Greenpeace werden auch auf lokaler Ebene sehr stark betont. Kooperationen mit anderen Gruppen werden sehr vorsichtig eingegangen. Bei sensiblen Themen wird auf eine einheitliche Außenwirkung geachtet (z.B. bei der „Südspange“, einem geplanten Umgehungsstraßenbau in Göttingen) und die Medienwirksamkeit steht bei Aktionen zum Teil stark im Vordergrund.

Viele der Punkte sind beim DJN nicht wiederzufinden. Da die Ziele der Organisation eher auf einen lokalen Wirkungsrahmen ausgerichtet sind und es stärker um das Lernen und Vermitteln der Inhalte durch die Mitglieder geht, spielen die Trends, die für große Umweltorganisationen gelten, kaum eine Rolle. Eine gewisse Professionalisierung liegt in der Herausgabe von botanischer und zoologischer Bestimmungsliteratur vor, die z.B. in Universitäten verwendet wird. Hier besteht eine Nische, in der der DJN eine hohe Kompetenz besitzt.

Eine Zunahme der Komplexität von Themen lässt sich in beiden Gruppen beobachten. Greenpeace folgt dem Trend und bindet lokale Themen in einen größeren Umweltzusammenhang ein, der wirtschaftliche und politische Fragen berücksichtigt. Der DJN engagiert sich auch in der globalen Klimapolitik, z.B. auf der Klimakonferenz in Kopenhagen 2009 oder dem McPlanet-Kongress 2009.

In Bezug auf Naturvorstellungen fällt zunächst die Begriffsbildung auf: In den Greenpeace-Interviews wurde vorrangig von "Umwelt" gesprochen, wohingegen in den DJN-Interviews eher "Natur" verwendet wurde. Darin spiegelt sich die Form der Aktivitäten wieder. Bei Greenpeace geht es darum, Unternehmen und Staaten durch gesellschaftlichen Druck zu einer Verhaltensänderung zu bewegen (z.B. weniger genetisch modifizierte Produkte zu produzieren oder den Walfang zu verbieten). „Umwelt" erscheint dabei als etwas, dass weiter entfernt ist (z.B. Abholzun- 
gen in den Tropen) oder eher einen technischen Bezug hat (z.B. genmodifiziertes Getreide, Kohlenstoffdioxidausstoß). Dieses Naturbild ist stark an einer modernen hochtechnisierten Industriegesellschaft orientiert, in der es um technische Entwicklung, Quantifizierbarkeit und Informationspolitik geht. Es wird sich auf bestimmte Kernthemen konzentriert, die medial gut verhandelbar und in der Öffentlichkeit und Politik präsent sind, wie z.B. Atomkraft oder Gentechnologie. Es dominiert dabei ein pragmatischer Ansatz im Sinne einer „Lösung“ für Umweltprobleme.

Im Gegensatz dazu ist „Natur“ im DJN etwas, dass erfahrbar oder erlebbar ist. Eine „Naturverbundenheit“ soll vermittelt werden und es geht um die Unterstützung von Umwelt- und Naturschutz. Hier zeigt sich auf der einen Seite ein Naturbild, das von den Begrifflichkeiten her an romantisierte Naturvorstellungen zu Beginn des 20. Jahrhunderts erinnert, auf der anderen Seite enthält es aber auch Elemente einer „bedrohten Natur“, z.B. durch Atomkraft, der 1960er Jahre. Besonders hervorgehoben wird im DJN das Lernen in Bezug auf „Natur“. Dies ist zum einen an individuellem Erleben und zum anderen an naturkundlichen Fakten orientiert und soll $\mathrm{zu}$ einer Bewusstseinsbildung für gesellschaftliche Veränderungen beitragen. Eine persönliche Auseinandersetzung mit „Natur" steht somit im Vordergrund.

Aus den Interviews und den Selbstbeschreibungen der Organisationen lässt sich entnehmen, dass verschiedene Naturvorstellungen vorherrschen: Auf der einen Seite eine eher rational-technische Betrachtung (z.B. Kohlenstoffdioxidbilanzen, Verunreinigungen der Ozeane durch Öl) bei Greenpeace und auf der anderen Seite eine individuell erfahrbare „Mitwelt“, deren Verständnis hilft, gesellschaftliche Veränderungen anzustoßen. In beiden Fällen geht es um gesellschaftliche Veränderungen, die aber über unterschiedliche Naturbegriffe kommuniziert werden.

\subsection{Einordnung in Zivilgesellschafts- und Partizipationstheorie}

Um Partizipation nicht offen im Raum stehen zu lassen, wurde das Konzept im Kapitel Partizipation (3.2) in zivilgesellschaftliche Theorien eingeordnet. Die beiden untersuchten Umweltorganisationen lassen sich als zivilgesellschaftliche Institutionen in dem Konzept von Pollak (2004) sehen (siehe 3.2.1). Sie sind nicht an staatliche Institutionen gebunden und handeln manchmal gegen deren Interessen. Um mit den gegebenen Mitteln auszukommen, müssen beide Organisationen wirtschaftlich handeln, als Non-Profit-Organisationen steht die Gewinnmaximierung allerdings nicht im Vordergrund. Dennoch ist gerade bei Greenpeace zu erkennen, dass die Sicherung von Wettbewerbsvorteilen im Wettkampf um Spendengelder und ein gutes öffentliches Image wichtig für den Erfolg der Organisation sind. Beide Organisationen tra- 
gen zur Bildung von sozialem Kapital bei. Hier wäre es interessant, mit weiteren Untersuchungen die zivilgesellschaftliche Einordnung und die Bildung von sozialem Kapital, insbesondere auf lokaler Ebene, zu erforschen. Es ist erkennbar, dass Greenpeace mit seinen zivilgesellschaftlichen Aktivitäten ein weites Feld aufspannt: vom Lobbying auf europäischer oder internationaler Ebene, bis hin zur Aufklärung von KonsumentInnen auf lokaler Ebene. Der DJN ist eher bestrebt, gesellschaftliche Perspektiven im Zusammenleben und -arbeiten aufzuzeigen, die durch den Staat oder andere zivilgesellschaftliche Organisationen nicht in dieser Form umgesetzt werden. Das wird z.B. durch die Abgrenzung zu Vereinen mit Erwachsenendachverbänden deutlich.

Ansätze aus den Partizipationstheorien finden sich in den Interviews wieder. Motivation und Barrieren, wie sie Oegema \& Klandermans (1994) für die Partizipation in sozialen Bewegungen und Organisationen nennen, finden sich in den Aussagen der InterviewpartnerInnen (z.B. hinsichtlich der verfügbaren Zeit oder der Erreichbarkeit der Treffen). Die Mobilisierungsbestrebungen der Organisationen konnten nicht im Detail untersucht werden. Hier bieten sich Anknüpfungen für weitere Forschungen an. Ferner ließ sich feststellen, dass Werte und Motive, die Form der Zusammenarbeit und die Alltagspraxen in den Organisationen, wie Penner (2004) sie nennt, zur einer besseren Partizipation beitragen. Das Rollenverhalten in den Organisationen konnte am Rande diskutiert werden, hier wäre eine Vertiefung in weiteren Studien, insbesondere im Hinblick auf die Verbindung von Rollenmustern und Verantwortung, interessant.

Die Arbeitsdefinition (siehe 3.4 hat sich für die Arbeit als praktikabel herausgestellt. Die ergänzenden Punkte fanden sich in den Interviews wieder und haben somit geholfen, ein umfassenderes Bild von der Sichtweise auf Partizipation durch die Interviewten zu bekommen. Dem Aspekt der „Interessengeleitetheit“ sollte noch eine Komponente der „Zielorientierung“ hinzugefügt werden. Dies beschreibt den Begriff etwas besser, da Partizipation mit bestimmten Zielsetzungen verknüpft ist. In den Interviews wurde immer wieder die Prozesshaftigkeit von Partizipation betont, dies lässt sich als ein allgemeines Kriterium festhalten und gilt nicht nur in Bezug auf Lern- und Entscheidungsprozesse.

\subsection{Forschungsfrage und Hypothesen}

Die Forschungsfrage (siehe 4.2.2 ließ sich im Laufe der Arbeit beantworten. Die Partizipation in den beiden Organisationen gestaltet sich unterschiedlich, allerdings gibt es einige grundlegende Faktoren, die sehr ähnlich sind, wie sich aus den Ergebnissen der Interviews, dem Vergleich mit der Theorie und der Einordnung in die Umweltbewegungen entnehmen lässt. Die Leitfragen haben dabei geholfen, das 
Thema zu erfassen. In dem Abschnitt Zusammenfassung der Ergebnisse (5.3) findet sich ein Überblick der zentralen Punkte aus den Interviews, die in die Diskussion der Hypothesen eingeflossen sind. Hinsichtlich des Geltungsbereiches der Hypothesen, sollte eine Übertragung der zentralen Ergebnisse auf andere Gruppen möglich sein. Die Hypothesen werden im Folgenden diskutiert.

\section{Es bestehen Unterschiede im Verständnis von Partizipation und deren Umsetzung zwischen den beiden Organisationen.}

Diese Hypothese lässt sich bestätigen. Zwar gibt es Gemeinsamkeiten im Verständnis von Partizipation (z.B. bezüglich der Assoziation mit „Teilnehmen“), aber im Detail werden Unterschiede sichtbar. Zwischen den Zielen und den Strukturen der Umweltorganisationen und dem Partizipationsverhalten gibt es einen Zusammenhang. Allerdings lässt sich keine kausale Beziehung darüber herleiten. Die Struktur der Organisationen bestimmt zum Teil darüber, wie zusammengearbeitet wird. Greenpeace schafft durch seine Struktur eine Form von Hierarchie und Leitung, durch die es bestimmte Aufgaben und Rollen in den Gruppen gibt. Der DJN versucht dies durch sein Selbstverständnis und durch seine Form der Zusammenarbeit zu vermeiden. Das hat Auswirkungen darauf, welche Personen sich in den Organisationen beteiligen und wie sich diese in den Organisationen selbst verorten. Ein gutes Beispiel ist hier der Umgang mit verschiedenen Altersgruppen: Greenpeace verfolgt einen eher pragmatischen Ansatz durch die Einteilung in Altersklassen. Der DJN versucht durch die Altersbeschränkung bewusst Hierarchien zu vermeiden. Die Sichtweise von Partizipation in Bezug auf die Zusammenarbeit mit Menschen verschiedenen Alters unterscheidet sich dadurch stark: Zusammenfassend lässt sich sagen, dass die Partizipation im DJN darauf ausgelegt ist, Selbstermächtigung und Eigeninitiative bei den Mitgliedern zu erreichen und ein kooperatives Zusammenleben zu ermöglichen. Demgegenüber versucht Greenpeace eher die Mitglieder in einen größeren Zusammenhang zu bringen, zu aktivieren und zu professionalisieren, um durch übergeordnete Strategien politischen und gesellschaftlichen Einfluss auszuüben.

\section{Die Faktoren, welche die Partizipation direkt beeinflussen, sind in beiden Organisationen ähnlich.}

Neben den Unterschieden im Verständnis von Partizipation gibt es einige grundlegende Gemeinsamkeiten, die förderlich für Partizipation sind. Obwohl es verschiedene Vorstellungen von „Natur" und „Umwelt“ gibt, ist ein allgemeines Interesse an diesen Themen und eine positive Einstellung zu Umwelt- und Naturschutz bei den Mitgliedern vorhanden. Hinzu kommt der Einsteig in die Umweltorganisationen, der häufig über Freunde und Bekannte erfolgte. Diese beiden Faktoren 
können als allgemeiner Zugang zum Feld der Umweltorganisationen gesehen werden. Barrieren, wie sie Oegema \& Klandermans (1994) beschrieben haben, sind einerseits die räumliche Entfernung zu den Treffen, die Partizipation behindern kann, und andererseits die Zeit, die für die Arbeit in den Organisationen zur Verfügung steht. Damit ist zum einen ganz allgemein die Bereitschaft gemeint, Zeit für ein Engagement aufzubringen und zum anderen die Zeit, die für die konkrete Mitarbeit eingeplant wird (daraus entstehen z.B. Probleme in Prüfungsphasen oder (Semester-)Ferien bei SchülerInnen und Studierenden). Eine funktionierende Kommunikation der Mitglieder untereinander und das Bewusstsein Verantwortung in den Gruppen zu übernehmen können als weitere Grundlagen für eine gelingende Partizipation gesehen werden. Diese erschienen in beiden Umweltorganisationen als relevant.

\section{Der DJN ist in seinen Aktivitäten eher nach innen gerichtet, Greenpeace eher nach außen.}

Beide Umweltorganisationen zielen darauf ab, gesellschaftliche Veränderungen zu erreichen. Die Unterschiede liegen in der Art und Weise, wie dies angegangen wird. Das Lernen, das gegenseitige Vermitteln von Wissen und die gemeinsamen Erfahrungen haben einen sehr hohen Stellenwert im DJN. Ihrem Motto nach „Wer die Welt verändern will, muss sie erst verstehen lernen!“ (DJN 2010a) setzen gesellschaftliche Veränderungen bei Individuen an. Greenpeace ist aktionsorientiert und versucht gesellschaftliche Veränderungen durch eine hohe Öffentlichkeitswirksamkeit und Medienpräsenz zu erreichen. Diese beiden Orientierungen fanden sich in den Interviews der entsprechenden Gruppen an vielen Stellen wieder. Die Innen- und Außenperspektive lässt sich auch in der Themenwahl wiederfinden. Bei Greenpeace werden überwiegend Themen aus deutschlandweiten oder internationalen Strategien aufgegriffen, wohingegen der DJN eher eigeninitiativ entwickelte Themen behandelt. Beide Umweltorganisationen grenzen sich ab: Greenpeace grenzt sich stark gegenüber staatlichen Institutionen und anderen Gruppen ab, um die eigene Unabhängigkeit zu wahren. Der DJN versucht eher eine interne Abgrenzung, in dem versucht wird, ein alternatives gesellschaftliches Miteinander zu finden, das mehr Selbstbestimmung und den Abbau von Hierarchien beinhaltet. Die Innen- und Außenperspektiven sind Bestandteil beider Gruppen. Insofern lässt sich die Hypothese nur teilweise bestätigen.

\section{Neue Hypothesen}

Durch die Auswertung war es möglich einige neue Hypothesen zu bilden, die in Hinblick auf die Fragestellung interessant erschienen und Raum für weitere Forschungen 
in diesem Bereich lassen. Auf weitere Forschungsfelder wird im Kapitel Reflexion und Fazit (7) hingewiesen. Als neue Hypothesen konnten formuliert werden:

- Es gibt verschiedene Partizipationsstrukturen in den jeweiligen Umweltorganisationen, diese sind abhängig von der organisatorischen Ebene (z.B. Bundesebene, internationale Ebene, Gruppenebene).

- Die Übernahme von Verantwortung ist eine Voraussetzung für eine gelingende Partizipation. Sie ist somit essentieller Bestandteil von Partizipationsprozessen.

- Der Einsatz von neuen Medien wird immer relevanter für die Partizipation in Umweltorganisationen.

- Es findet eine Professionalisierung in der freiwilligen Arbeit statt. Diese betrifft auch kleine Umweltorganisationen. 


\section{Reflexion und Fazit}

In diesem abschließenden Kapitel geht es um die Diskussion und Reflexion von inhaltlichen, theoretischen und methodischen Aspekten, um den Forschungsprozess in seiner Gesamtheit transparenter zu machen. Dabei werden der Forschungsstand (1.2), die Ziele der Untersuchung (4.2.1) und die Gütekriterien (4.1.3) für die qualitative Forschung aufgegriffen. Das Kapitel schließt mit einem Fazit und Ausblick.

\subsection{Inhaltliche und theoretische Reflexion}

Die Forschungsfrage ließ sich im Laufe der Arbeit größtenteils beantworten. Es war möglich, die im Vorhinein aufgestellten Hypothesen zu bestätigen und neue Hypothesen zu entwickeln. Ein nächster Schritt wäre es, die Forschungsfrage weiter zu differenzieren und Unteraspekte näher zu untersuchen. In Hinblick auf die Theorie hat sich gezeigt, dass es nur wenige Ansätze gibt, die sich explizit mit der Partizipation in Organisationen beschäftigen. Eine stärker interdisziplinäre Verknüpfung wäre hier wünschenswert. Das Verhältnis von Partizipation und Zivilgesellschaft wurde, als Reaktion auf die geringe gesellschaftstheoretische Einbettung, in dieser Arbeit andiskutiert, sollte allerdings noch vertieft werden. Daraus ergeben sich zwei Perspektiven auf den Partizipationsbegriff: zum einen Partizipation als eine analytische Kategorie, die es ermöglicht gesellschaftliche Prozesse zu untersuchen und zum anderen als ein normatives Konzept auf der Ebene der konkreten Zusammenarbeit. Dies betrifft insbesondere die Frage, wie Partizipation in Gruppen gelingen kann. Die Einordnung der Umweltorganisationen in das weitere Feld der sozialen Bewegungen konnte hier nur relativ allgemein umgesetzt werden. Dieser Punkt ließe sich, in Hinblick auf Partizipation als Grundlage sozialer Bewegungen mit ihrem gesellschaftlichen und politischen Einfluss, noch vertiefen. Eine noch stärker sozialgeographische Betonung wäre in der Untersuchung von regionalen Unterschieden in der Partizipation auf lokaler Ebene oder in der Untersuchung von Natur- und Umweltvorstellungen als Motive für die Partizipation möglich. Dies hätte jedoch den Rahmen dieser Arbeit überschritten. Weitere Forschungsfelder eröffnen sich ferner in der stärker biographisch ausgerichteten Erforschung des Partizipationsverhaltens, in der Bedeutung des Internets und social media für die Partizipation und der Mobilisierung der Mitglieder der Organisationen auf lokaler Ebene. Die Ziele 
der Untersuchung (siehe 4.2.1) konnten erfüllt werden und es ergeben sich daraus Anknüpfungen für die weitere Forschungstätigkeit. Der Stand der Forschung (siehe 1.2 kann um eine weitere qualitative Arbeit ergänzt werden. Da keine quantitativen Studien auf einer lokalen Ebene vorzufinden waren, konnte dahingehend kein Vergleich vorgenommen werden.

\subsection{Methodische Reflexion}

Die Arbeit hatte einen methodischen Schwerpunkt. Es wurde Wert darauf gelegt, den theoretischen Anteil nicht zu vernachlässigen und durch Dokumentation des Auswertungsprozesses und inhaltliche, methodische und theoretische Kontrolle (siehe 4.1.3) die Nachvollziehbarkeit zu gewährleisten. Die Mischung aus explorativer Arbeit, in der Leitfragen und Hypothesen als „roter Faden“ dienen, hat sich als praktikabel erwiesen. Das Vorwissen ist insbesondere in die Erstellung des Interviewleitfadens und in die Bildung der Hypothesen eingeflossen. Die Fallauswahl (siehe 4.2.3) war für eine explorative Studie gut geeignet, auch wenn einige Interviewtermine nicht zu Stande kamen. Die InterviewpartnerInnen spiegeln ein relativ breites Spektrum in Bezug auf das Alter und die Aktivität in den Organisationen wieder. Trotzdem wäre es gut gewesen, wenn Interviews mit der JAG von Greenpeace zustande gekommen wären, um die Bedeutung des Altersunterschieds in den Organisationen besser herausarbeiten zu können. Die Aufnahme und Auswertung von sechs Interviews erschien vom Aufwand her für eine sechsmonatige Arbeit als angemessen. Für die Auswertung hätte es Vorteile gehabt, wenn Erhebung und Auswertung der Interviews stärker ineinander geflossen wären, um prozessorientierter forschen zu können. Der Einsatz von offenen, halbstrukturierten Interviews mit narrativen Elementen als Forschungsmethode war gut umsetzbar. Für die weitere Forschung in diesem Bereich wäre es sinnvoll, diese durch quantitative Methoden, im Sinne einer methodischen Triangulation, zu ergänzen. Dies würde es ermöglichen, die Aussagekraft der Hypothesen noch zu vertiefen.

\subsection{Fazit und Ausblick}

Umweltorganisationen müssen mit ihren Themen und Strategien auf die sich ständig verändernden politischen und gesellschaftlichen Rahmenbedingungen reagieren. Das geht nicht ohne die Berücksichtigung der Befindlichkeiten und Fähigkeiten der Mitglieder in den Organisationen, die sich durch freiwilliges Engagement einbringen. Ziel dieser Arbeit war es, die Partizipation, also die freiwillige und unbezahlte Beteiligung von Personen in den Umweltorganisationen Greenpeace und dem Deutschen Jugendbund für Naturbeobachtung (DJN), zu untersuchen. Dabei hat sich herausgestellt, 
dass es Unterschiede und Gemeinsamkeiten im Verständnis von dem, was Partizipation ausmacht, zwischen diesen beiden Organisationen gibt. Die Ziele für das Engagement und die Strukturen in den Organisationen unterschieden sich sehr voneinander, allerdings ließ sich feststellen, dass die Grundvoraussetzungen für den Einstieg in das Feld der Umweltorganisationen und mögliche Hindernisse ähnlich sind. Die Arbeit liefert damit Erkenntnisse darüber, wie Partizipation in Umweltorganisationen auf lokaler Ebene abläuft, wie sich die Mitwirkung in Entscheidungsprozessen ausgestaltet und was hinderlich und förderlich für Partizipation ist. Der Ansatz der Arbeit war es dabei, durch qualitative Interviews die individuellen Perspektiven der Mitglieder über ihre Mitwirkung in den Organisationen aufzuzeigen.

Für die Umweltorganisationen wird das Thema Partizipation auch in Zukunft eine hohe Relevanz haben. Es gibt Schwankungen in den Mitgliederzahlen und es werden weiterhin Themen angegangen werden, die gesellschaftlich bedeutsam sind, und es bilden sich neue Kooperationen und Netzwerke zwischen Organisationen. Letztendlich können die Umweltorganisationen diese Aufgaben nicht ohne das freiwillige Engagement ihrer Mitglieder bewältigen und sie sind darauf angewiesen, deren Erwartungen und Wünsche zu berücksichtigen. Partizipation kann es ermöglichen, gesellschaftliche Veränderungen zu bewirken, die vom Staat nicht berücksichtigt werden. Sie kann Teil der persönlichen Entwicklung sein und besitzt eine politische Wirkkraft. In diesem Sinne soll mit einem Zitat von Stange (2004: 10) geendet werden:

„Der Gedanke der Partizipation wird von vornherein verfälscht, wenn seine Verwirklichung einer Gesellschaft aufgezwungen wird. Partizipation ist nur vorstellbar als permanenter Lernprozess möglichst vieler Menschen; sie lässt ,Zukunft offen' und ermöglicht die freie Diskussion über Zukunftsalternativen." 


\section{Literatur}

Adloff, F. (2005): Zivilgesellschaft - Theorie und politische Praxis. Campus Verlag: Frankfurt (Main)

Baukloh, A. \& Roose, J. (2002): The Environmental Movement and Environmental Concern in Centemporary Germany. In: Goodbody, A. (Hrsg.): The Culture of German Environmentalism - Anxieties, Visions, Realities. Berghahn Books: New York, Oxford

Bekkers, R. (2005): Participation in Voluntary Associations: Relations with Resources, Personality, and Political Values. Political Psychology, Vol. 26(3): 439454

Benford, R. D. (1992): Social Movements. In: Borgatta, E. F. \& Borgatta, M. L. (Hrsg.): Encyclopedia of Sociology [S-Z]. 10. Aufl., Macmillan Publishing Company: New York

BERLIN.DE (2010): Tag des Ehrenamtes. URL: http://www.berlin.de/ ba-charlottenburg-wilmersdorf/org/planleit/tag_des_ehrenamtes. html [26.08.2010]

Bliss, F. \& Neumann, S. (2008): Participation in International Development Discourse and Practice - SState of the Artänd Challenges. INEF Report 94, Institute for Development and Peace, Universität Duisburg-Essen

Blühdorn, I. (2002): Green Futures? A Future for the Greens?. In: Goodbody, A. (Hrsg.): The Culture of German Environmentalism - Anxieties, Visions, Realities. Berghahn Books: New York, Oxford

Brand, K.-W. (2008): Umweltbewegung (inkl. Tierschutz). In: Roth, R. \& Rucht, D. (Hrsg.): Die sozialen Bewegungen in Deutschland seit 1945 - Ein Handbuch. Campus Verlag: Frankfurt (Main)

BrunnengräBer, A. \& WALK, H. (2001): NGOs unter Ökonomisierungs- und Anpassungsdruck - Die Dritte-Sektor-Forschung und ihr Beitrag zur Analyse des NGO-Phänomens. In: BrunnengräBer, A.; Klein, A. \& Walk, H. (Hrsg.): 
NGOs als Legitimationsressource: zivilgesellschaftliche Partizipationsformen im Globalisierungsprozess. Leske + Budrich: Opladen

Bühlmann, M. (2005): Politische Partizipation im kommunalen Kontext: der Einfluss lokaler Kontexteigenschaften auf individuelles politisches Partizipationsverhalten. Dissertation Universität Bern

Cohn, S. F.; Barkan, S. E. \& Halteman, W. A. (2003): Dimensions of Participation in a Professional Social-Movement Organization. Sociological Inquiry, Vol. 73(3): 311-337

Cooke, B. \& Kothari, U. (Hrsg., 2004): Participation - The new Tyranny?. Zed Books: London, New York

Dahm, D. \& Scherhorn, G. (2008): Urbane Subsistenz - Die zweite Quelle des Wohlstands. Oekom Verlag: München

DelBRÜCK, J. (2003): Nichtregierungsorganisation - Geschichte - Bedeutung Rechtsstatus. Rechspolitisches Forum 13, Institut für Rechtspolitik, Universität Trier

Diekmann, A. \& Preisendörfer, P. (2001): Umweltsoziologie - Eine Einführung. Rowohlt Taschenbuch Verlag: Hamburg

DJN (2010): Satzung des Deutschen Jungendbundes für Naturbeobachtung. DJN: Steinhude

DJN (2010a): Der Verband. URL: http://naturbeobachtung.de/verband [30.08.2010]

DJN (2010b): Geschäftsordnung des Deutschen Jungendbundes für Naturbeobachtung. DJN: Rotenburg

DJN (2010c): Publikationen und Shop. URL: http://naturbeobachtung.de/ publikationen [30.08.2010]

DJN (2010d): DJN-Ortsgruppe Göttingen. URL: http://naturbeobachtung.de/ ortsgruppen/goettingen [30.08.2010]

Duden (2000): Duden - die deutsche Rechtschreibung. 22. Aufl., Dudenverlag: Mannheim

Dunn, K. (2000): Interviewing. In: Hay, I. (Hrsg.): Qualitative Research Methods in Human Geography. Oxford University Press: Victoria 
Club of Rome (1979): Das menschliche Dilemma - Zukunft und Lernen. 3. Aufl., Verlag Fritz Molden: Wien et al.

Embacher, S. \& LANG, S. (2008): Bürgergesellschaft - Eine Einführung in zentrale bürgerschaftliche Gegenwarts- und Zukunftsfragen. Verlag J.H.W. Dietz: Bonn

Enquette-Kommission (2002): Bürgerschaftliches Engagement: auf dem Weg in eine zukunftsfähige Bürgergesellschaft. Drucksache 14/8900, EnquetteKommission „Zukunft des bürgerschaftlichen Engagements“ des Deutschen Bundestages

EYV 2011 (2010): European Year of Volunteering 2011. URL: http://www. eyv2011.eu [31.08.2010]

FliCK, U. (2007): Qualitative Sozialforschung - Eine Einführung. Rowohlt Verlag: Hamburg

Flick, U. (2008): Design und Prozess qualitativer Forschung. In: FLICK, U.; vON Kardorff, E. \& Steinke, I. (Hrsg.): Qualitative Forschung - Ein Handbuch. Rowohlt Taschenbuch Verlag: Hamburg

Freeman, R. B. (1997): Working for Nothing: The Supply of Volunteer Labour. Journal of Labour Economics, Vol. 15(1): 140-166

Gensike, T. (2006): Bürgerschaftliches Engagement in Deutschland. In: BpB (Hrsg.): Bürgerschaftliches Engagement. Aus Politik und Zeitgeschichte 12/2006, Bundeszentrale für politische Bildung Bonn

GläSER, J. \& LAUdel, G. (2009): Experteninterviews und qualitative Inhaltsanalyse. 3. Aufl., VS Verlag für Sozialwissenschaften: Wiesbaden

Goodbody, A. (2002): Anxities, Visions and Realities: Environmentalism in Germany. In: Goodbody, A. (Hrsg.): The Culture of German Environmentalism - Anxities, Visions, Realities. Berghahn Books: New York, Oxford

GreEnPEACE (2008): Greenpeace structure. URL: http://www.greenpeace.org/ international/en/about/how-is-greenpeace-structured/ [30.08.2010]

Greenpeace (2009): Annual Report 2009. Greenpeace International

Greenpeace (2010): Fragen \& Antworten zu Greenpeace. URL: http: //www.greenpeace.de/ueber_uns/nachrichten_ueber_uns/artikel/ fragen_antworten_zu_greenpeace-1/ [30.08.2010] 
GreenPeace (2010a): Greenpeace Gruppe Göttingen. URL: http://gruppen. greenpeace.de/goettingen/ [26.08.2010]

GreenPeace (2010b): Mitmachen. URL: http://www.greenpeace.de/ueber uns/mitmachen/ [26.08.2010]

Grobe, R. (2006): Vom Ehrenamt zum Bürgerschaftlichen Engagement - Tendenzen im Naturschutz. In: BFN (Hrsg.): Freiwilligenarbeit im Naturschutz. Naturschutz und biologische Vielfalt 37, Bundesamt für Naturschutz Bonn

GöRG, C. (1999): Gesellschaftliche Naturverhältnisse. Verlag Westfälisches Dampfboot: Münster

Helfferich, C. (2005): Die Qualität qualitativer Daten - Manual für die Durchführung qualitativer Inverviews. 2. Aufl., VS Verlag für Sozialwissenschaften: Wiesbaden

Hermanns, H. (2008): Interviewen als Tätigkeit. In: Flick, U.; von KARdorfF, E. \& Steinke, I. (Hrsg.): Qualitative Forschung - Ein Handbuch. 6. Aufl., Rowohlt Taschenbuch Verlag: Hamburg

Hirsch, J. (2001): Des Staates neue Kleider - NGO im Prozess der Internationalisierung des Staates. In: Brand, U.; Demirovic, A.; Görg, C. \& Hirsch, J. (Hrsg.): Nichtregierungsorganisationen in der Transformation des Staates. Westfälisches Dampfboot Verlag: Münster

JAeggi, E.; FAAs, A. \& Mruck, K. (1998): Denkverbote gibt es nicht! Vorschlag zur interpretativen Auswertung kommunikativ gewonnener Daten. Forschungsbericht 2, Abteilung Psychologie im Institut für Sozialwissenschaften, Technische Universität Berlin

Jensen, B. B. (2002): Knowledge, Action and Pro-environmental Behaviour. Environmental Education Research, Vol. 8(3): 325-334

Kern, T. (2008): Soziale Bewegungen - Ursachen, Wirkungen, Mechanismen. VS Verlag für Sozialwissenschaften: Wiesbaden

Kersting, N. (2008): Politische Beteiligung - Einführung in dialogorientierte Instrumente politischer und gesellschaftlicher Partizipation. In: Kersting, N. (Hrsg.): Innovative Partizipation: Legitimation, Machtkontrolle und Transformation. Eine Einführung. VS Verlag für Sozialwissenschaften: Wiesbaden

Klandermans, B. \& Oegema, D. (1987): Potentials, Networks, Motivations, and Barriers: Steps Towards Participation in Social Movements. American Sociological Review, Vol. 52(4): 519-531 
Klein, A. \& Löw, M. (2006): Ausmaß und Potential des freiwilligen Engagements im Bereich Natur- und Umweltschutz in Deutschland. In: BFN (Hrsg.): Freiwilligenarbeit im Naturschutz. Naturschutz und biologische Vielfalt 37, Bundesamt für Naturschutz Bonn

KockA, J. (2003): Zivilgesellschaft in historischer Perspektive. Forschungsjournal NSB, Vol. 16(2): 29-37

LAMnEK, S. (1995): Qualitative Sozialforschung - Band 2: Methoden und Techniken. 3. Aufl., Beltz Verlag: Weinheim

LAmneK, S. (2005): Qualitative Sozialforschung - Lehrbuch. 4. Aufl., Beltz Verlag: Weinheim, Basel

Markham, W. T. (2004): Überleben in schwierigen Zeiten - Deutsche Umweltorganisationen im 20. Jahrhundert. artec-paper 112, artec - Forschungszentrum Nachhaltigkeit, Universität Bremen

Markham, W. T. (2008): Environmental Organisations in Modern Germany Hardy Survivors in the Twentieth Century and Beyond. Berghahn Books: New York, Oxford

Martinez, T. A. \& McMullin, S. L. (2004): Factors Affecting Decisions to Volunteer in Nongovernmental Organizations. Environment and Behavior, Vol. 36(1): 112-126

Mayring, P. (2000): Qualitative Inhaltsanalyse. Forum: Qualitative Sozialforschung, Vol. 1(2): 1-10

Mayring, P. (2002): Einführung in die qualitative Sozialforschung. 5. Aufl., Beltz Verlag: Weinheim, Basel

Mayring, P. (2007): Qualitative Inhaltsanalyse - Grundlagen und Techniken. 9.. Aufl., Beltz Verlag: Weinheim, Basel

Mayring, P. (2008): Qualitative Inhaltsanalyse. In: Flick, U.; von KardorfF, E. \& Steinke, I. (Hrsg.): Qualitative Forschung - Ein Handbuch. Rowohlt Taschenbuch Verlag: Hamburg

Meinefeld, W. (2008): Hypothesen und Vorwissen in der qualitativen Sozialforschung. In: Flick, U.; von KARdorfF, E. \& Steinke, I. (Hrsg.): Qualitative Forschung - Ein Handbuch. 6. Aufl., Rowohlt Taschenbuch Verlag: Hamburg 
Mitlacher, G. \& Schulte, R. (2005): Steigerung des ehrenamtlichen Engagements in Naturschutzverbänden. BfN-Skripten 129, Bundesamt für Naturschutz Bonn

Moser, S. (2010): Beteiligt sein. Partizipation aus der Sicht von Jugendlichen. VS Verlag für Sozialwissenschaften: Wiesbaden

Nassmacher, H. (2002): Politikwissenschaft. 4. Aufl., Oldenbourg: München, Wien

Nolte, P. (2003): Zivilgesellschaft und soziale Ungleichheit. Forschungsjournal NSB, Vol. 16(2): 38-45

Oegema, D. \& Klandermans, B. (1994): Why Social Movement Sympathizers Don’t Participate: Erosion and Nonconversion of Support. American Sociological Review, Vol. 59(5): 703-722

Offe, C. \& Fuchs, S. (2001): Schwund des Sozialkapitals? Der Fall Deutschland. In: Putnam, R. (Hrsg.): Gesellschaft und Gemeinsinn - Sozialkapital im internationalen Bereich. Verlag Bertelsmann Stiftung: Gütersloh

Pain, R. (2004): Social Geography: Participatiory Research. Progress in Human Geography, Vol. 28(5): 652-663

Penner, L. (2002): Dispositional and Organizational Influences on Sustained Volunteerism - An Interactionist Perspective. Journal of Social Issues, Vol. 58(3): $447-467$

Pollak, D. (2004): Zivilgesellschaft und Staat in der Demokratie. In: KLEIN, A.; Kern, K.; Geissel, B. \& Berger, M. (Hrsg.): Zivilgesellschaft und Sozialkapital - Herausforderungen politischer und sozialer Integration. VS Verlag für Sozialwissenschaften: Wiesbaden

Robbins, Paul, H. J. \& Moore, S. A. (2010): Environment and Society. WileyBlackwell: Chichester et al.

Roberts, J. M. \& Devine, F. (2004): Some Everyday Experiences of Voluntarism: Social Capital, Pleasure, and the Contingency of Participation. Social Politics, Vol. 11(2): 280-296

Rosenbladt, B. VON (Hrsg., 2001): Freiwilliges Engagement in Deutschland Ergebnisse der Repräsentativerhebung zu Ehrenamt, Freiwilligenarbeit und bürgerschaftlichem Engagement. 2. Aufl., Verlag W. Kohlhammer: Stuttgart 
Saur, Wolfgang (2002): 100 Jahre Wandervogel: Geschichte - Deutung Wirkung. 43. Jahrestagung der Gesellschaft für Geistesgeschichte vom 1. 3. November 2001 in Berlin-Steglitz. Zeitschrift für Religions- und Geistesgeschichte, Vol. 54(2): 171-178

Schmidt, M. G. (2004): Wörterbuch zur Politik. 2. Aufl., Kröner Verlag: Stuttgart

Stange, W. (2008): Was ist Partizipation? Definitionen - Systematisierungen. Baustein A 1.1. URL: http://www.kinderpolitik.de/beteiligungsbausteire/ grundlagen.php [23.08.2010]

Steinke, I. (2008): Gütekriterien qualitativer Forschung. In: FLICK, U.; VON KARDorfF, E. \& Steinke, I. (Hrsg.): Qualitative Forschung - Ein Handbuch. 6. Aufl., Rowohlt Taschenbuch Verlag: Hamburg

SZ (2009): Kraftloses Zwitschern. URL: http://www.sueddeutsche.de/ wirtschaft/twitter-und-kein-ende-kraftloses-zwitschern-1.32016 [06.09.2010]

SZ (2010): Zwitschern gehört zum Handwerk. URL: http://www.sueddeutsche. de/medien/digitaler-journalismus-zwitschern-gehoert-zum-handwerk-1. 971334 [06.09.2010]

Torgler, B. \& Garcia-Valiñas, M. A. (2006): Participation in Environmental Organizations: An Empirical Analysis. Working Paper 2006-06, Center for Research in Economics, Management and the Arts (CREMA) Basel

Voss, K. (2009): Öffentlichkeitsarbeit von Nichtregierungsorganisationen - Mittel Ziele - intere Strukturen. VS Verlag für Sozialwissenschaften: Wiesbaden

Wallraff, B. (2010): Professionelles Management von Ehrenamtlichen - Eine empirische Studie am Beispiel von Greenpeace Deutschland. Budrich UniPress: Opladen, Farmington Hills

Winchester, H. P. M. (2000): Qualitative Research. In: HAy, I. (Hrsg.): Qualitative Research Methods in Human Geography. Oxford University Press: Victoria

Zeiler, C. \& Schell, S. (2003): Partizipation und nachhaltige Entwicklung - Partizipation von Mädchen und jungen Frauen in Umweltverbänden in Östereich. URL: www.gap-europe.de/Sprachen/home_deutsch/Studien/ umwelt_au.pdf [23.08.2010]

ZEIT (2010): Alle Freunde auf einen Klick. URL: http://www.zeit.de/digital/ internet/2010-08/soziale-netzwerke-freunde [06.09.2010] 


\section{A Anhang}

\section{A.1 Interviewleitfaden „Partizipation in Umweltorganisationen“}

\section{Einstieg / Zusammenarbeit}

- Kannst du die Situation beschreiben, wie es war als du angefangen hast?

- Wo engagierst du dich?

- Wie bist du dazu gekommen?

- Wie läuft es da ab? Wie arbeitet ihr zusammen?

- Wer bestimmt?

- Was kann deiner Meinung nach helfen gut zusammenzuarbeiten?

- Fällt dir ein positives oder negatives Beispiel zu eurer Zusammenarbeit ein?

\section{Motivation}

- Hast du dich früher auch schon engagiert?

- Wo und wie?

- War das in den Gruppen unterschiedlich?

- Was ist deine Motivation dich zu engagieren?

- Wie siehst du dich selbst in der Organisation?

\section{Partizipation}

- Was bringt dein Engagement anderen?

- Was bringt dein Engagement dir?

- Kannst du mit dem Begriff Partizipation etwas anfangen?

- Wenn du anderen den Begriff erklären solltest, wie würdest du ihn umschreiben?

- Muss man Partizipation erlernen? 
- Kann das Jede/r?

- Wie könntet ihr eine größere Beteiligung erreichen?

\section{Natur- und Umweltschutz}

- Wie positioniert ihr euch deiner Meinung nach in Umwelt- und Naturschutz?

- Welche aktuellen Entwicklungen fallen dir auf?

\section{Abschluss}

- Was macht Greenpeace/DJN für dich besonders?

- Was denkst du könnt ihr in Zukunft bewirken?

- evtl. Frage nach Alter, Schule/Ausbildung/Studium

- Willst du noch etwas sagen?

- Möchtest du noch etwas über mich wissen?

- Fragen oder Anmerkungen per Mail oder Telefon 


\section{A.2 Überblick über die Interviews}

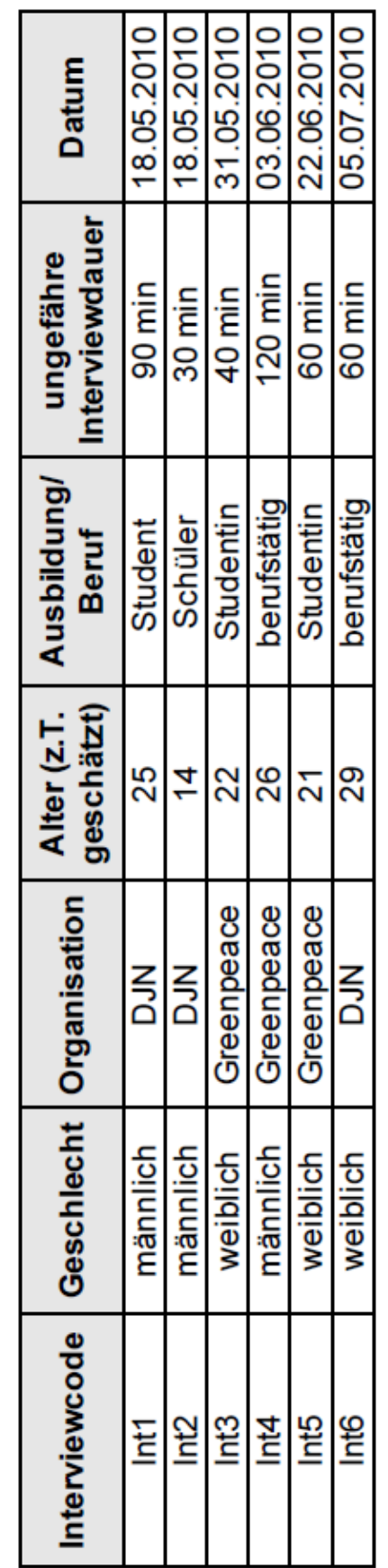

Abbildung A.1: Interviewte Personen 


\section{A.3 Inhaltsverzeichnis der CD}

Auf der beiliegenden CD-ROM befinden sich die Transkripte der Interviews (der Dateiname besteht aus dem Interviewcode und dem Datum des Interviews) und eine digitale Version dieser Arbeit.

- Int1_100518.pdf

- Int2_100518.pdf

- Int3_100531.pdf

- Int4_100603.pdf

- Int5_100622.pdf

- Int6_100705.pdf

- Engbers_2010_Partizipation_in_Umweltorganisationen.pdf 
